Portland State University

PDXScholar

Spring 5-16-2014

\title{
Design and Prototyping of an Antenna-Coupled Cryotron
}

Shauna Jensen

Portland State University

Follow this and additional works at: https://pdxscholar.library.pdx.edu/open_access_etds

Part of the Power and Energy Commons

Let us know how access to this document benefits you.

\section{Recommended Citation}

Jensen, Shauna, "Design and Prototyping of an Antenna-Coupled Cryotron" (2014). Dissertations and Theses. Paper 1788.

https://doi.org/10.15760/etd.1787

This Thesis is brought to you for free and open access. It has been accepted for inclusion in Dissertations and Theses by an authorized administrator of PDXScholar. Please contact us if we can make this document more accessible: pdxscholar@pdx.edu. 


\title{
Design and Prototyping of an Antenna-Coupled Cryotron
}

by

Shauna Marie Jensen

A thesis submitted in partial fulfillment of the requirements for the degree of

\author{
Master of Science \\ in \\ Electrical and Computer Engineering
}

Thesis Committee:

Robert Bass, Chair

Richard Campbell

Branimir Pejcinovic

Portland State University

2014 
(C) 2014 Shauna Marie Jensen 


\section{Abstract}

Grid-scale integration of renewable energy sources and smart grid devices has created new demands in flexible power conversion. State-of-the-art semiconductor power switches present limitations in power handling capability, as well as forward and reverse breakdown voltages. Superconducting materials are a viable alternative due to their robustness against high ampacities, large electric fields and abrupt changes in power flow. This work pays focus to material testing and apparatus design for an antenna-coupled cryotron (ACC), which is a superconducting power switch.

Design, fabrication and testing are examined for a longitudinal resonant cavity, paired with monopole transmit and modified slot receive antennae. These couple radio frequency (RF) energy into superconducting thin film niobium $(\mathrm{Nb})$ carrying high current densities $\left(\sim 105 \mathrm{~A} / \mathrm{cm}^{2}\right)$, thereby creating an antenna-coupled cryotron.

Induced electromagnetic field effects at the receive antenna alter superconductive fluid dynamics. The theorized quality in manipulating this mechanism is a rapid normal-conductivity transition $(\mu s)$, which affects a switch "off" state. Functional evaluation of the device as a waveguide revealed evanescent mode resonance at frequencies below the waveguide cut-off of $\sim 18 G H z$. The thin film $\mathrm{Nb}$ was deposited on a quartz dielectric, which penetrated the waveguide and supported evanescent resonances within the structure. 
Altered resistivity and critical transition-point properties emerged from device testing at applied RF. When the $\mathrm{Nb}$ film temperature-dependent coherence length was comparable to its thickness, perpendicular magnetic field application generated an Abrikosov vortex state, energetically favoring a mixed domain condensate. Interaction of the magnetically-induced flux vortex lattice with Lorentz current forces gave rise to resistive changes within the metal. Three resistive transition mechanisms developed: a latch to normal state resistance, attributed to cooper-pair destruction avalanche induced near critical transition points; a small reversible increase in resistance $(\sim m V)$, arising from flux-flow within an intermediate state at peak resonance; as well as temporal alterations in superfluid dynamics from disequilibrium in the quasi-particle population. The RF-induced superfluid effects were observable in separate terms of electric and thermodynamic fluctuations.

Motivation for this work is the eventual design of a high voltage, high current and low cost power switch, able to function where existing semiconductor technology fails. Concentration is paid to the fundamental theory, physics and methodology in conceptual testing and design of prototype ACCs. Assessment focuses on preliminary findings and concludes with next stage design requirements. 


\section{Acknowledgements}

I would like to gratefully acknowledge the collaborators on this project: Robert Bass $\stackrel{1}{\square}$ Aaron Datesman, Aric Datesman and Arthur Lichtenberger; as well as thesis committee members: Richard Campbell and Branimir Pejcinovic. It has been an honor to participate in this work and gain research experience under such thoughtful and insightful minds as theirs.

\footnotetext{
${ }^{1}$ Robert Bass is also the graduate advisor and thesis committee chair for this project.
} 


\section{Contents}

Abstract i i

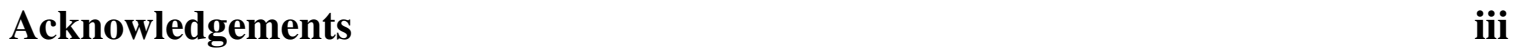

List of Figures vii

List of Tables $\quad$ xii

1 Introduction 1

1.1 Background . . . . . . . . . . . . . . . . 1

1.2 Theory . . . . . . . . . . . . . . . . . . 3

1.2.1 Superconductivity and Normal State Transition . . . . . . . . . . 3

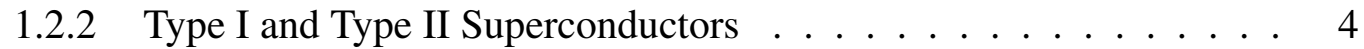

1.2.3 Antenna Coupling . . . . . . . . . . . . . . . 6

1.2 .4 Quasi-Particle Redistribution . . . . . . . . . . . . . . . 7

\begin{tabular}{|l|l|l}
2 & Design & 11
\end{tabular}

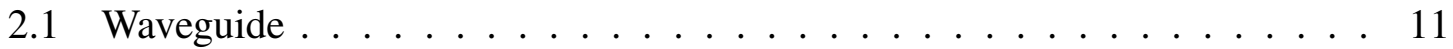

2.1 .1 Mode Selection . . . . . . . . . . . . . . . . . . . 11

2.1 .2 Geometry Design . . . . . . . . . . . . . . . . . . . . . . . . . . . . . . . . 14

2.1.3 Frequency Selection . . . . . . . . . . . . . . . . 17

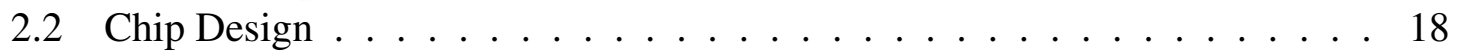

2.2 .1 Dielectric . . . . . . . . . . . . . . . . . 18

2.2 .2 Antennae ............................ 20

2.2 .3 Metallization ...................... 22

2.2.3.1 Nb Transmission Substrate . . . . . . . . . . . . 22

$2.2 .3 .2 \mathrm{Au}$ Contact Pads . . . . . . . . . . . . . 23

2.3 HFSS Simulation . . . . . . . . . . . . . . . . 23

2.3 .1 Waveguide Function . . . . . . . . . . . . . . 23

2.3 .2 Parametric Sweeps and Sensitivity Analysis . . . . . . . . . . . . . . 26

2.3 .3 HFSS Animation Stills . . . . . . . . . . . . . . . . . . . 33

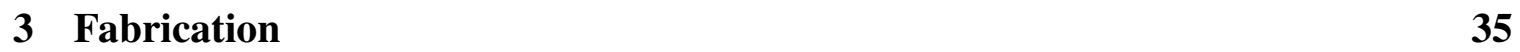

3.1 Sample Mounting on Si Carrier . . . . . . . . . . . . . . . . . . 36

3.2 RCA Cleaning . . . . . . . . . . . . . . . . . 37 
3.3 Evaporation and Deposition Processes for CEMN Machines. . . . . . . . . 39

$3.4 \mathrm{Au}$ Contact Pad Photolithography and Wet Etch . . . . . . . . . . . . . . . 40

3.4 .1 Photolithography . . . . . . . . . . . . . . . 40

3.4 .2 Au Etching . . . . . . . . . . . . . . . . . . . . . . . . . . . . . . . . . . 42

$3.5 \mathrm{Nb}$ Etch Techniques $\ldots \ldots \ldots$. . . . . . . . . . . . . . . 43

3.5 .1 Wet Etch . . . . . . . . . . . . . . . . . . . . . . . . . . . . . . . 44

3.5 .2 Reactive Ion Etch . . . . . . . . . . . . . . . 46

\begin{tabular}{|lll}
4 & Experimental Methods & 54
\end{tabular}

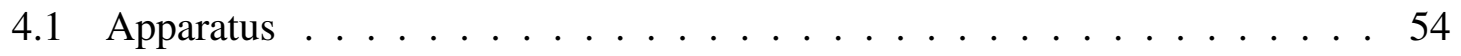

$4.1 .1 \quad$ Dipstick . . . . . . . . . . . . . . . . 55

4.1.2 Auxiliary Elements for Process Control . . . . . . . . . . . . . . . 60

4.1.2.1 Temperature Cycling . . . . . . . . . . . . . 61

4.1.2.2 Thermal Conductivity . . . . . . . . . . . 62

$4.1 .2 .3 \quad$ Electrical Isolation . . . . . . . . . . . . . . . . . . . . . . . . . . . 63

4.2 Calibration and Diagnostics . . . . . . . . . . . . . . . . . 63 63

4.2 .1 Temperature Sensor . . . . . . . . . . . . . . . . . . . 63

4.2 .2 Sources and Meters . . . . . . . . . . . . . . . . . . 64

4.2 .3 LHe Level Evaluation . . . . . . . . . . . . . . . . . . . . 65

4.3 Testing Processes $\ldots \ldots \ldots \ldots 6$. . . . . . . . . . . . . . . . . . . . . . . . . . . 66

$4.3 .1 \quad$ ACC Chip Variation . . . . . . . . . . . . . . . . . . . . . . 66 6

4.3 .2 Base Critical Current - Full Submersion . . . . . . . . . . . . . . . 67

4.3 .3 Current Sweep Profile. . . . . . . . . . . . . . . . . . . . . . . 68

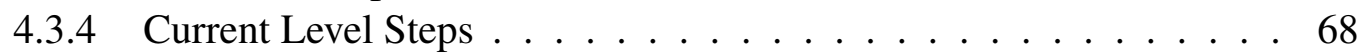

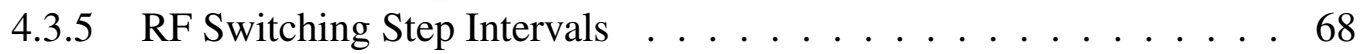

4.3 .6 Transmit Antenna Placement . . . . . . . . . . . . . . . . . . . 69

4.3 .7 Temporal Trends Near $I_{C} \ldots \ldots \ldots$. . . . . . . . . . . . . . . . . . . . . . . 70

4.3 .8 Frequency Effect Sweeps . . . . . . . . . . . . . . . . . . . . 70

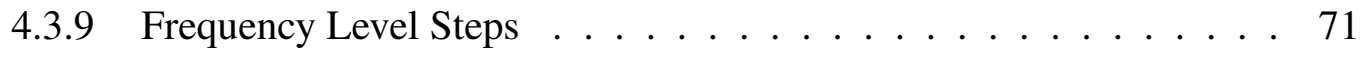

4.3 .10 Temperature Effect Steps . . . . . . . . . . . . . . . . . . . 71

4.3 .11 Temperature Tracking . . . . . . . . . . . . . . . . . . . 72

4.3 .12 Power Level Evaluation . . . . . . . . . . . . . . . . . . . . 72

4.3 .13 Waveguide Alteration . . . . . . . . . . . . . . . . 72

4.3 .14 Shunt Impedance . . . . . . . . . . . . . . . . . . . . . . . . . . . . . . . . . 73

4.3 .15 S11 at Room Temperature . . . . . . . . . . . . . . 73

$\begin{array}{lll}5 & \text { Results } & \mathbf{7 5}\end{array}$

5.1 Quasi-particle Redistribution . . . . . . . . . . . . . . . . . 75

5.1.1 Temporal Trends Near $I_{C} \ldots \ldots \ldots$. . . . . . . . . . . . 76

5.2 Flux Lattice Effects . . . . . . . . . . . . . . . . . . . . . . . 79

$5.2 .1 \quad$ Establishment of Switching Condition . . . . . . . . . . . . . 79

5.2 .2 Normalized Configuration Resistance Comparisons . . . . . . . . . 84

$5.2 .2 .1 \quad$ Receive Antenna . . . . . . . . . . . . . 85 
5.2 .2 .2 Quartz Dielectric . . . . . . . . . . . . . . . 86

5.2 .2 .3 Altered Waveguide . . . . . . . . . . . . . . 88

$5.2 .2 .4 \quad$ Coaxial Transmit Extension . . . . . . . . . . . . . . . 90

5.2 .3 Frequency-Induced Voltage and Temperature . . . . . . . . . . 91

5.2 .4 Shunt Path for Latched Switching $\ldots \ldots \ldots$

$5.2 .5 \quad$ Swept Power Level $\ldots \ldots \ldots$. . . . . . . . . . . . . . . . . . . 99

6 Discussion 101

6.1 Post-Testing HFSS Mode1 . . . . . . . . . . . . . . . . . . . . . 101

6.2 Hypotheses for Results . . . . . . . . . . . . . . . . . . . . . 103

6.3 Waveguide-Induced Quasi-Particle Redistribution . . . . . . . . . . . . 104

6.4 Abrikosov Flux-Flow Resistance . . . . . . . . . . . . . . . . . . . . . . 107

6.4 .1 Development of Dielectric Resonance . . . . . . . . . . . . 107

6.4 .2 Abrikosov Vortex . . . . . . . . . . . . . . . . . . . . 113

6.4 .3 Flux-flow Resistive Voltage. . . . . . . . . . . . . . . . 115

\begin{tabular}{lll}
\hline 7 & Conclusion & 117
\end{tabular}

7.1 Findings . . . . . . . . . . . . . . . . . . . . . 117

7.1 .1 Quasi-Particle Redistribution . . . . . . . . . . . . . . . 117

$7.1 .2 \quad$ Flux-Flow . . . . . . . . . . . . . . . . . . . 118

7.2 Further Work … . . . . . . . . . . . . . . . . . 118

$7.2 .1 \quad$ Quasi-Particle Disruption Experimentation . . . . . . . . 118

7.2 .2 Flux-Flow Engineering $\ldots \ldots \ldots \ldots$

\begin{tabular}{ll}
\hline Bibliography & 122
\end{tabular}

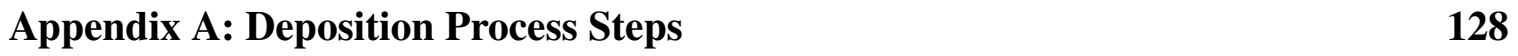

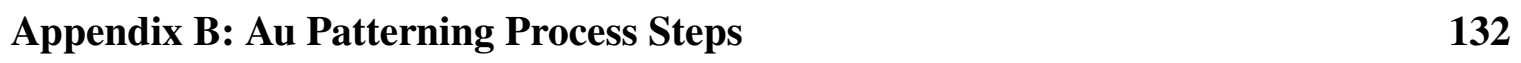

Appendix C: HF Handling Safety for Nb Etch Procedures 135

\begin{tabular}{ll}
\hline Appendix D: Nb RIE Process Algorithm & 137
\end{tabular}

\begin{tabular}{ll}
\hline Appendix E: Temperature Calibration & 140
\end{tabular}

Appendix F: Thin Strip-Line Current Sweep Temperature Depression 143 


\section{List of Figures}

1.1 Comparative plots between type I and type II superconductors, illustrating spatial variance of the energy order and magnetic flux penetration. Notably, the magnetic flux penetration depth for a type II is much larger than that of a type I, showing the difference in respective abilities to support partial magnetism while retaining energy state stability. Coherence length of a type II is thus much smaller than that of a type $\mathrm{I} . \ldots \ldots \ldots \ldots$

2.1 Traditional quarter-wave high field waveguide layout and equivalent circuit. The purpose of the circuit is to create optimal coupling and directivity between transmit and receive ports. . . . . . . . . . . . . . . 12

2.2 Induced current within $\mathrm{Nb}$ due to high E-field orientation. The left waveguide image illustrates circular longitudinal $\mathrm{H}$-field components, with an $\mathrm{H}$-null and lateral E-maximum in the center of the structure. Subsequent ACC chip coupling effects are shown on the right. $\ldots \ldots \ldots \ldots \ldots$

2.3 Illustrative layout of the waveguide geometry with major design parameters

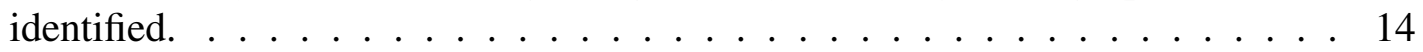

2.4 Smith chart tracking of the functional quality provided by tuning stubs, BS1 and BS2. Rather than quarter-wave high-field caps, they were optimized in HFSS

for impedance matching between the antennae. . . . . . . . . . . . . . 24

2.5 Equivalent circuit schematic and functional values at the optimized design frequency, for quasi-particle redistribution, of $19.8 \mathrm{GHz}$. . . . . . . . . . 25

2.6 Parametric analysis, sweeping monopole antenna length. Minimal S11 occurred with parameter values: $B S 1=3.89 \mathrm{~mm}, B S 2=6.35 \mathrm{~mm}(0.25$ "), monopole $_{\text {length }}=3.14 \mathrm{~mm}$, slot $_{\text {length }}=3.63 \mathrm{~mm}$, slotwidth $=2.94 \mathrm{~mm}$, and $Q t z_{\text {slug }}=0.35 \mathrm{~mm}$. These parameters correspond with a frequency of $19.8 \mathrm{GHz} \ldots \ldots \ldots \ldots \ldots \ldots$

2.7 Parametric monopole transmit sweeps, with the quartz dielectric adjusted to a thickness of $0.515 \mathrm{~mm}$. The originally specified $3.14 \mathrm{~mm}$ extension maintained minimal S11, but the optimal frequency was reduced to $18.75 \mathrm{GHz} . \quad$. . . . . . 29

2.8 S11 analysis incorporating extremely low sheet resistance $\left(S R=8 e^{-6} \Omega /\right.$ square $)$ of the $\mathrm{Nb}$ sample, with all other design variables held constant. The S11 characteristic within the waveguide frequency range showed varied sensitivity to extremely low sheet resistance. . . . . . . . . . . . . . . . . 31 
2.9 Swept $\mathrm{Nb}$ sheet resistance data at $19.8 \mathrm{GHz}$ showed sufficient robustness against large error margins, with a maximum S11 at $-4.8 d B$. The $\mathrm{x}$-axis is shown in log scale to account for swept orders of magnitude in the variable. . . . . . . . 32

2.10 E-field animation at $19.8 \mathrm{GH} z$ showed a large longitudinally travelling electric wave. It carried an intensity of $\sim 2 \mathrm{~V} / \mathrm{mm}$, which was consistent with design

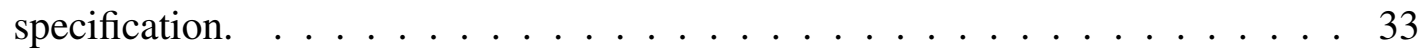

2.11 Induced surface current density profile at $19.8 \mathrm{GHz}$ indicated a strong induced

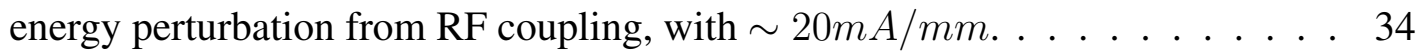

3.1 Illustrative representation of fully deposited ACC chip before patterning of the

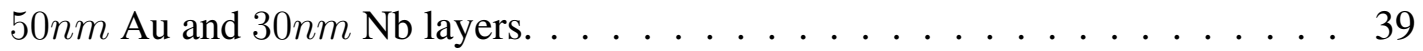

3.2 ACC01 Au Mask (shown to-scale), used for lithographic creation of probe contact pads. . . . . . . . . . . . . . . . . 40

3.3 Illustrative representation of the patterned $50 \mathrm{~nm}$ Au layer after photolithography and etching. . . . . . . . . . . . . . . . . . . 42

3.4 ACC02 Nb Mask (shown to-scale), used to create the base design patch receive

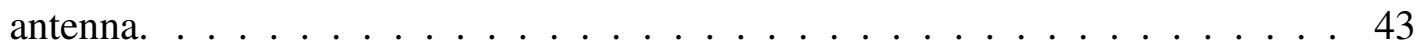

3.5 ACC03 Nb Trim Mask (shown to-scale), used to constrict current flow regions for tests with reduced $J_{C} \ldots \ldots \ldots \ldots \ldots \ldots$

$3.6 \quad$ Illustrative representation of the fully patterned ACC chip after final RIE on the $30 n m$ Nb layer. . . . . . . . . . . . . . . . . . . 52

3.7 Fully fabricated ACC chip, shown in the copper holding stage of the device testing apparatus. .................. 53

$4.1 \quad$ Illustrative model of dipstick testing apparatus with all major components visible. 54

4.2 Back view of EMI/RFI shield box with connection terminals of the current feed, voltage probe, RF input and temperature sensor shown. . . . . . . . . . . . . . 55

4.3 Inside view of the EMI/RFI shield box input terminals connected to feed wires; which were led into the metal conduit through a rubber stopper for stability. . . 56

4.4 Side view of the full assembly, showing four-point ACC probe wires feeding into the $\mathrm{Au}$ probes, held in the macor blocks on either side of the waveguide. Below the $\mathrm{Cu}$ holding stage, wires were fed to the DT670A-BO. . . . . . . . . 57

4.5 Back view of the ACC assembly, showing BS1 and the DT670A-BO. . . . . . . 58

4.6 Top view of the ACC assembly, shown with the top cap on BS2 removed in order to reveal the inner waveguide. $\ldots \ldots \ldots \ldots$. . . . . . . . . . 59

4.7 Machining CAD schematics for the ACC. Drawn by Aaron Datesman and machined by Aric Datesman . . . . . . . . . . . . . . . 60

4.8 Assembly CAD schematics for the ACC. Drawn by Aaron Datesman. . . . . . 60

5.1 Induced voltage comparison at the design frequency in close range of $I_{C}$ indicated signs of energy gap depression, which is a function of even-mode energy perturbation in quasi-particles. . . . . . . . . . . . . . 76

5.2 Temperature profile for the $1.47 \mathrm{~A}$ application evidencing energy gap depression. 77 
5.3 Induced voltage comparison at the design frequency sufficiently far below $I_{C}$ indicated signs of energy gap enhancement, a function of even-mode energy perturbation in quasi-particles. . . . . . . . . . . . . . . 78

5.4 Temperature profile for the $1.445 \mathrm{~A}$ application evidencing energy gap enhance-

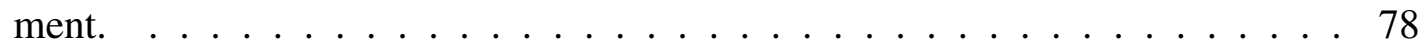

5.5 Bar graph comparison of normalized resistance values at $15 \mathrm{GHz}$ with appliedand absent- RF radiation. The total transmit monopole antenna extension was

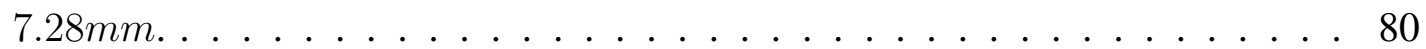

5.6 OFF-ON-OFF-ON RF switching progression comparison of normalized resistance values at $15 \mathrm{GHz}$ established that the voltage development was reversible. The total transmit monopole antenna extension was $7.28 \mathrm{~mm}$. . . . . . . . . . . 81

5.7 Establishment of the dominant voltage developing frequency at $4 \mathrm{GHz}$ with a $7.28 \mathrm{~mm}$ total transmit monopole antenna extension. . . . . . . . . . . . . 82

5.8 Temperature dependence of voltage level and reversibility at the dominant voltage developing frequency of $4 \mathrm{GHz}$ with a $7.28 \mathrm{~mm}$ total transmit monopole

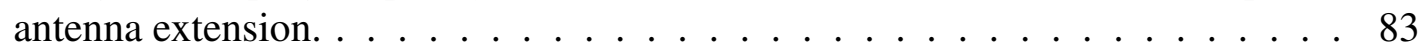

5.9 A comparison plot of all testing configurations showed deviations of scale in resistance levels caused by varied conditions. The two cases with highest resistance levels incorporated the $7.28 \mathrm{~mm}$ monopole extension and restricted transmission width, respectively. . . . . . . . . . . . . . . 84

5.10 Comparative normalized resistance profiles for varied antenna configurations. Similar resistance levels were observed for trimmed $\mathrm{Nb}$ transmission width and the elongated monopole extension of $7.28 \mathrm{~mm}$. Comparison between the $7.28 \mathrm{~mm}$ monopole extension and the $5.15 \mathrm{~mm}$ monopole extension showed much higher relative resistance with the former. . . . . . . . . . . . 85

5.11 Identical testing parameters with variance only in the quartz showed similar coupling for the two quartz samples. The crystalline quartz displayed higher temperature stability than the amorphous sample. . . . . . . . . . . . 86

5.12 Measurements of a thin strip-line transmission geometry compared to $\mathrm{Nb}$ transmission surfaces incorporating the slot antenna show drastic deviation. The results indicate that the slot antenna is necessary for inducing a voltage within the superfluid. . . . . . . . . . . . . . . . 87

5.13 The removal of BS1 during testing, with all other variables held constant, did not show significant changes in normalized resistance levels. A small reduction in resistance without BS1 is likely a product of direct exposure of the quartz and transmit monopole to LHe, lowering the thermodynamic energy in the slug. 88

5.14 The removal of BS2 during testing, with all other variables held constant, showed a significant increase of normalized resistance at $4 \mathrm{GHz}$; and also a

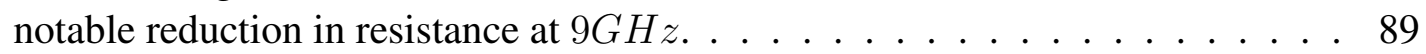

5.15 Focused representation of discrepancies in normalized resistance profile, only in reference to transmit monopole extension. . . . . . . . . . . . . . . . 90 
5.16 Isolated frequency applications with a $7.28 \mathrm{~mm}$ monopole transmit antenna extension. Values were calculated from an average of 11 samples taken over a $\sim 5 s$ total time interval. . . . . . . . . . . . . . . . . 91

5.17 Temperature tracking correspondent with isolated frequency applications at a $7.28 \mathrm{~mm}$ monopole transmit antenna extension. Values were calculated from an average of 11 samples taken over $\mathrm{a} \sim 5 s$ total time interval. . . . . . . . . . 92

5.18 Induced voltage over sequentially swept frequencies, with $100 \mathrm{M} \mathrm{Hz}$ resolution, at $7.28 \mathrm{~mm}$ monopole transmit antenna extension. Samples were taken as single datum, at a rate of 1 sample per $100 \mathrm{~ms} . \quad \ldots \ldots . . \ldots 93$

5.19 Temperature tracking correspondent with sequentially swept frequencies, with $100 \mathrm{MHz}$ resolution, at $7.28 \mathrm{~mm}$ monopole transmit antenna extension. Samples were taken as single datum, at a rate of 1 sample per $100 \mathrm{~ms} . \quad$. . . . . . . . 94

5.20 Outlier exclusion of Figure 5.19 revealed frequency-induced thermodynamic changes in the $6 G H z$ and $9 G H z-12 G H z$ range. . . . . . . . . . . . . . 94

5.21 Excessively high energy levels within the ACC created thermal runaway, which caused latched switching events. . . . . . . . . . . . . 95

5.22 Equivalent Circuit for shunt load testing configuration. . . . . . . . . . . . . . 96

5.23 Shunt-impedance-controlled RF switching at an excessively high energy level maintained relative stability. . . . . . . . . . . . . . 97

5.24 Normalized coupling resistance for swept power levels, comparatively applied to four separate testing scenarios. . . . . . . . . . . . . . . . 99

6.1 Post testing HFSS device maximum longitudinal E-field analysis at $4 \mathrm{GHz}$. A travelling field was expected along the z-axis for the waveguide to support any given frequency; which did not occur at $4 G H z$. . . . . . . . . . . . . . 102

6.2 Post testing HFSS device maximum H-field analysis at $4 \mathrm{GHz}$. A null field at the quartz was expected for the device to function as designed. Rather, the

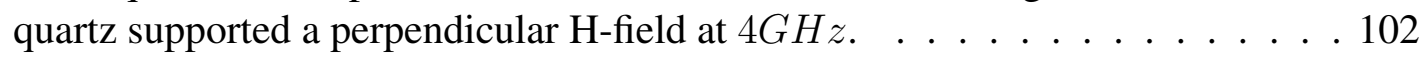

6.3 $\quad$ Post testing HFSS device maximum surface current density at $4 \mathrm{GHz}$, with reduced sheet resistance to account coupling into a superfluid. . . . . . . . . . 103

6.4 Sheet impedance parameter sweeps, accounting for the reduced material impedance in the superconducting state. The reduced material impedance creates a higher S11. These sweeps were produced with an adjusted quartz chip, which led to coupling at low frequency levels. . . . . . . . . . . . . . . . 106

6.5 Sequential parametric sweeps of the transmit sleeve monopole extension length confirm that placements allowing longer transmit segments within the waveguide cause low S11 at reduced frequency levels. . . . . . . . . . . . . . . . . . 109

6.6 Equivalent circuit schematics and functional values at the observed induced voltage peaks of $4 G H z$ and $9 G H z . \ldots \ldots \ldots \ldots$

6.7 Field representation of the slot-line effect on the quartz dielectric for evanescent mode resonance. . . . . . . . . . . . . . . . . 112 
F.1 Current sweep voltage and temperature profiles for B-MS-FT, showing trends consistent with standard expectations. . . . . . . . . . . . . . . . . 145

F.2 Current sweep voltage and temperature profiles for B-TL-0, showing wire cooling with increased applied current; as well as a current density level far

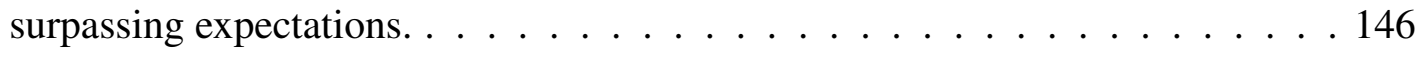




\section{List of Tables}

2.1 Initial mode property specifications at $20 \mathrm{GHz}$, assuming vacuum propagation parameters $\epsilon_{0}$ and $\mu_{0}$. TM $\mathrm{TM}_{01}$ propagation constant $(\beta)$ and guided wavelength $\left(\lambda_{g}\right)$ are imaginary values, indicating the mode is cut off at this frequency... .18

$3.1 \quad \mathrm{Nb}$ RIE Recipe for $410 \mathrm{~cm}^{2}$ Platen at $21^{\circ} \mathrm{C} \ldots \ldots \ldots$. . . . . . . . . . . . . 49

4.1 Physical Properties of Tested Samples. Common to all samples are metal deposition thicknesses of $30 \mathrm{~nm} \mathrm{Nb}$ and $50 \mathrm{~nm} \mathrm{Au}$; as well as area dimensions

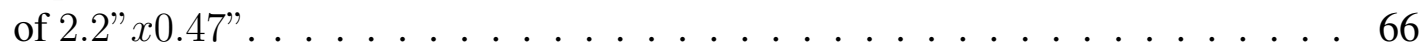

4.2 Conditions and Examination per Test Scenario. Examination techniques are identified with their corresponding subsection heading numbers. . . . . . . . . 74

5.1 Shunt Load Sharing at $4 G H z$ applied RF and Antenna Extension of $7.28 \mathrm{~mm}$, using chip A-MS-FT. . . . . . . . . . . . . . . . 98 


\section{Chapter 1: Introduction}

The initial purpose of this project was to establish proof-of-concept for a theorized switching mechanism, using quasi-particle redistribution in a superconducting $\mathrm{Nb}$ strip. During the experimentation process, a discovery was made for an altered device function. Within the altered operation, a flux-flow resistive voltage was supported in the $\mathrm{Nb}$. This voltage was both higher in magnitude and more easily controlled than the initial phenomenon under test.

The original design was based on coupling transmit and receive antennae within a waveguide. When functioning in an altered capacity, the device acted instead like an evanescent mode dielectric resonant filter. During this operation the $\mathrm{Nb}$ transitioned to a mixed normal-superconducting regime. Applied current, at a sufficiently high level, transformed stationary flux within the metal to a mobile state; thus inducing the observed voltage..$^{2}$

\subsection{Background}

The present necessity for technological advancements in switching operations on the bulk electric power grid is increasing at a rapid pace. Capabilities in power handling, switching speed, reverse power flows, heightened power levels and flow control must evolve to meet

\footnotetext{
${ }^{2}$ The initial theory and design of the ACC device, in Chapters 1 and 2, pays focus to quasi-particle redistribution goals. Chapter 6 then explains the observed altered functionality, using techniques similar to those developed in Chapters 1 and 2.
} 
new issues posed by renewable energy, the networked smart grid, stochastic generation and consumption, and transactive markets. Grid-interactive components, like inverters, charge controllers, and a host of flexible AC transmission (FACTS) devices rely on switching operations that are carried out on semiconductor materials. These switches carry limitations, all related to the needs of the developing grid.

The U.S. Department of Energy has enacted a research and development (R\&D) plan for material upgrades in power switching technology; with explicitly stated targets in peak current capabilities, breakdown voltages, switching speeds and switching losses. [38] The progress of material advancement for semiconductors is reaching saturation levels, thus superconductors present an alternative to reach R\&D upgrade targets. Superconductors carry advantages over semiconductors in simplicity of fabrication, forward/reverse breakdown voltages and current carrying capabilities. The goal of designing a switching mechanism based in superconductive materials is to create a robust and reliable power switch, operational in an evolved power grid.

ACC valves have potential application in high voltage, high current power switching converter technologies; including STATCOMs, unified power flow controllers and utilityscale inverters. A small array of four cross-connected valves would create a perfectly bi-directional switch that is flexibly controllable. ACCs are not subject to the forward or reverse breakdown voltages that limit the use of semiconductor-based power switches. 


\subsection{Theory}

This section covers the basis of the two major contributing phenomena involved with ACC design, superconductivity and antenna coupling.

\subsubsection{Superconductivity and Normal State Transition}

Superconductivity defines a property that some materials are capable of adopting when subject to a specific set of external conditions. The major factor governing superconductive ability is temperature. When superconductors are cooled below a temperature threshold, known as the critical temperature, the mass properties of electrons within the material change.[49] Rather than acting like discrete interactive masses, they retain only the element of carrying force, and consist of the matter that binds mass together. [20, 49] In this state electrons display an intermediate phase in particle-wave duality. They are no longer particles, but also do not function as a unified wave. Electrons in this condition are called quasiparticles. They are classified as fermions and carry a half-integer atomic spin, and as such, must occupy separate energy states.[49] The separation in energy states causes an inability for quasi-particles to act like a unified waveform; and also leaves them subject to kinetic energy loss.

Quasi-particles that are depressed to sufficiently low energy levels will attract one another. They communicate across the atomic material lattice to form pairs, through elastic phonon bonding.[49] These coupled quasi-particles are called cooper-pairs. [49] They are bosons and carry an integer value atomic spin. [49] Cooper-pairs are able to occupy a common 
energy state without limit. They are not defined by quantized particle movement, but instead move as an elastic collective excitation that mimics the action of a single particle. [20, 49] This causes uniformity in the current flow on the material, because the entirety of valence electrons are bound at the same Fermi energy level.[49] Cooper-pairs act like a wave and are not subject to kinetic losses. From this arises the unique advantage of superconductors, to transport massive amounts of current without resistive losses.

The transition from a superconducting state to a normal state involves overcoming an energy threshold, called the Fermi energy gap. [49] It is defined as the amount of energy needed to break cooper-pairs back into quasi-particles. A fully normal transition must then convert quasi-particles into normal electrons. Methods of achieving superconducting-tonormal transition use elevated levels of current, temperature or external energy perturbations like radiant electromagnetic fields.[49, 53]

\subsubsection{Type I and Type II Superconductors}

Pertinent to the results of this project is a distinction between the two different types of superconductors. The ACC transmission strip-line design was based on type I superconductivity. However, due to $\mathrm{Nb}$ deposition thickness and experimental biasing conditions, it instead adopted properties of a type II. $3^{3}$

A superconductor containing free energy within a specific range near the transition point enters a state where both cooper-pairs and quasi-particles exist together. This creates a two-fluid model of superconductivity. When this occurs, a domain energy wall forms

\footnotetext{
${ }^{3}$ Further interpretation in Chapter 6.
} 
between the two species. With a type I material, the domain wall has positive energy and completely separates the two fluids. The domain wall for a type II superconductor has negative energy, which creates a void in the energy balance that favors mixing of the two fluids. [20, 49]

A type I superconductor adheres strictly to the Meissner effect, which is to say that it expels and fully excludes magnetic flux from the interior of the material while superconducting. This phenomenon is known as diamagnetism. It creates a wholly superconducting regime until very near the critical normal transition point. An abrupt transition occurs when the magnetic flux completely penetrates into the material. In contrast, a type II material allows partial magnetic flux penetration, which begets a mixed superconducting-normal state and causes a gradual superconducting-to-normal transition.[49]
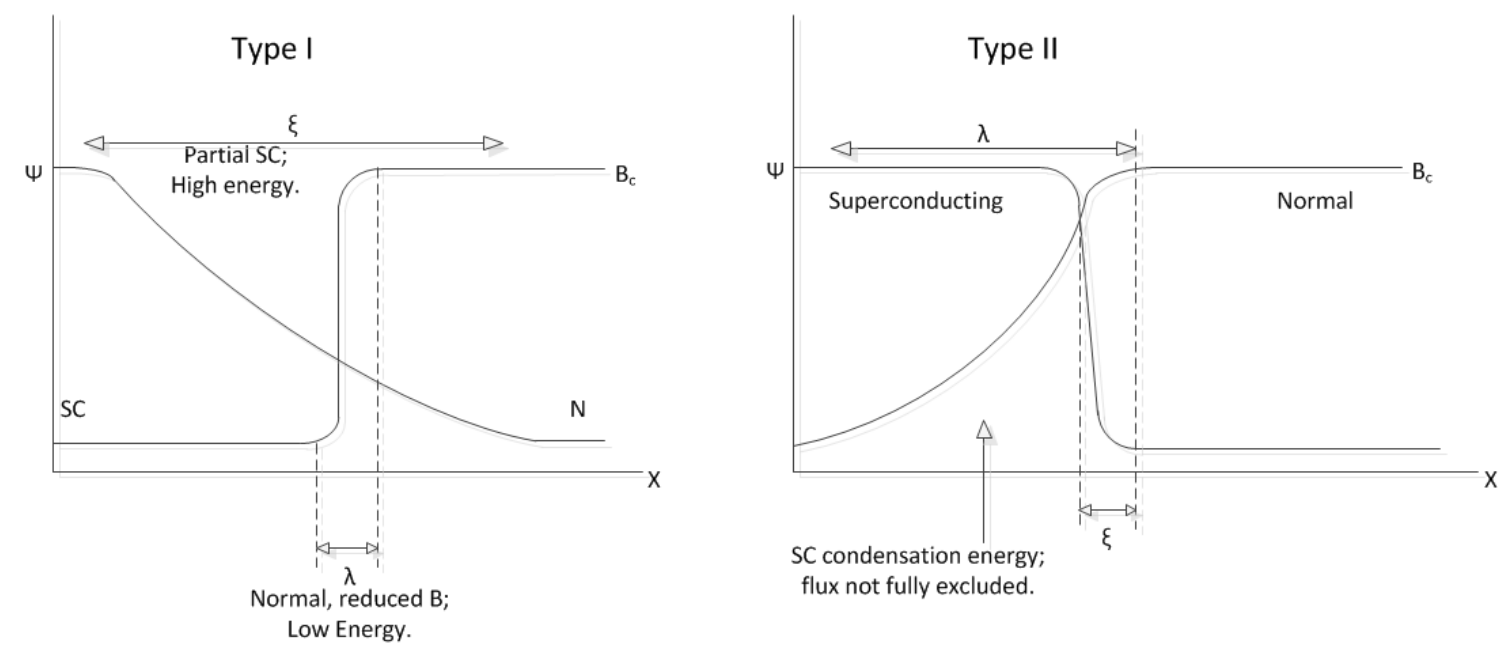

Figure 1.1: Comparative plots between type I and type II superconductors, illustrating spatial variance of the energy order and magnetic flux penetration. Notably, the magnetic flux penetration depth for a type II is much larger than that of a type I, showing the difference in respective abilities to support partial magnetism while retaining energy state stability. Coherence length of a type II is thus much smaller than that of a type I. 
Figure 1.1 shows the differences between type I and type II superconductors graphically. Here, $\lambda$ refers to the magnetic flux penetration depth of the material $\left.\right|^{4}$ and $\xi$ is the coherence length, which is a spatial parameter of energy state stability. $\Psi$ is the energy order (or cumulative wave function), $B_{c}$ is the critical normal magnetic flux, and the x-axis represents spatial distance. Notable differences shown in these plots are that the spatial variance in energy order and magnetic flux are opposite for the two types. Inverted magnetic penetration depth and coherence length are also apparent by comparison.

\subsubsection{Antenna Coupling}

The notion for antenna coupling of the ACC was derived from heterodyne mixers for radio astronomy telescopes. [4, 15, 30] Coupling in these devices is based on high frequency signals ( $\mathrm{THz}$ ) with energy levels capable of breaking cooper-pairs and spurring a fully normal transition. The ACC design was purposed for lower frequency levels $(G H z)$ with only enough energy to disturb the quasi-particle population.5 The initial antenna dimensions were based on quarter-wave high-field transmit and receive matching.[3, 41] Ideally, the energy input to the superfluid would be a function only of the receive antenna geometry. With a receive antenna patterned into the $\mathrm{Nb}$ strip-line, an external energy perturbation could be established. This is favorable over current- and temperature-induced transitions, in terms of power switching, because it is more controllable and requires less energy input.

\footnotetext{
${ }^{4}$ In subsequent chapters $\lambda$ will reference wavelength. It is illustrated here as the magnetic flux penetration depth to maintain consistency with literature on the subject.

${ }^{5}$ Because cooper-pairs are comprised of two bound quasi-particles, the energy required for their destruction is much higher than that for quasi-particle disruption.
} 


\subsubsection{Quasi-Particle Redistribution}

With an external energy perturbation, the quasi-particles occupying low energy states are elevated to higher energy levels.[49] This elevation leaves open spaces for new quasiparticles at the lowest energy states above the Fermi energy gap, which is the energy threshold to transform cooper-pairs to quasi-particles. $]^{6}$ When quasi-particles occupy all of the low energy states, it is less probable that cooper-pairs will break, since they cannot immediately occupy the closest energy states as quasi-particles. [49] The ACC engineering design involved quasi-particle redistribution to higher energy states, leaving low states open for broken cooper-pairs. The process of redistributing quasi-particles would cause a thermodynamic change in the superfluid, which would allow easier destruction of cooperpairs.

Relaxation time in a superconductor is the interval for the state to settle to equilibrium after an energy perturbation. It is defined by the amount and rate of particle scattering caused by the input energy. With an external perturbation that overcomes the relaxation rate of the superfluid, disequilibrium ensues. Two effects of disequilibrium occur in these states, known as even and odd mode.[49]

Even mode disequilibrium is mainly associated with perturbations by neutral particles, like phonons, photons and radiant waves; odd mode is induced by charged particles, like electrons. Even and odd mode can be excited conjointly with charged particles as well. It is important to note that odd mode will not arise through exposure to neutral particles. This is to say that mere exposure of a superconducting material to microwave radiation in a

\footnotetext{
${ }^{6}$ Akin to the energy gap required in semiconductor devices.
} 
vacuum may create even mode quasi-particle disequilibrium; but electromagnetic coupling for inducing charge separation within fields is necessary for odd mode development. An antenna, patterned within the superconducting substrate, serves to induce both forms of disequilibrium. The odd mode is of highest interest because it induces voltage, which can be used to control switching. The even mode is also useful because it can change the energy gap required for breaking cooper-pairs. The following set of equations define these processes.[49]

BCS Energy Gap Equation:

$$
\frac{2}{V}=\sum_{k} \frac{1-2 f_{k}}{E_{k}}=\sum_{k} \frac{1-2 f_{k}}{\left(\triangle^{2}+\xi_{k}^{2}\right)^{1 / 2}}
$$

where,

$E_{k}=$ Excitation per particle in energy state, $k$

$f_{k}=$ Quasi - particle occupation probability function for energy state, $k$ $\triangle=$ Energy gap parameter

$\xi=$ Energy of a single electron at state, $k$ 
Fermi Thermal Equilibrium:

$$
f_{0}\left(\frac{E_{k}}{k T}\right)=\left[1+e^{E_{k} / k T}\right]^{-1}
$$

where,

$f_{0}=$ Quasi - particle occupation probability function at normal state Fermi level

$k=$ Boltzmann constant

$T=$ Temperature

Quasi-particle Population Change:

$$
\delta f_{k} \equiv f_{k}-f_{0}\left(\frac{E_{k}}{k T}\right)
$$

where,

$\delta f_{k}=$ Deviation in quasi - particle occupation probability function

$$
\text { for energy state, } k
$$

Deviation from thermal equilibrium (Even Mode):

$$
\frac{\delta T^{*}}{T} \equiv \frac{T^{*}-T}{T} \approx \frac{1}{N(0)} \sum_{k} \frac{\delta f_{k}}{E_{k}}=\int_{-\infty}^{\infty} \frac{\delta f_{k}}{E_{k}} d \xi_{k}
$$

Charge imbalance (Odd Mode):

$$
Q^{*} \equiv q_{k} \delta f_{k}=\sum_{k} \frac{\xi_{k}}{E_{k}} \delta f_{k}
$$


Equations 1.1 through 1.5 denote mathematical representations of energy changes caused by external energy perturbations. Importantly, $\delta f_{k}$ is created as a result of quasi-particle redistribution and contributes to both even and odd mode effects. Thus, quasi-particle redistribution can create a voltage through odd mode imbalance, and also change the energy gap magnitude with even mode. 


\section{Chapter 2: Design}

\subsection{Waveguide}

A waveguide was designed to enclose the send and receive antennae of the $\mathrm{ACC}$, in order to maximize coupling between the two. Waveguides operate much like single conductor transmission lines, supporting either electric wave propagation or magnetic wave propagation along the longitudinal axis.[41] Cross sectional dimensions govern allowable frequencies within the structure, while the length defines a longitudinal field profile. When the length dimensions on either side of the send-receive circuit are equal to a quarter wavelength of the design frequency, it creates open circuit high-field conditions at the antenna nodal boundaries.[10, 40, 41]

The quarter-wave open circuit impedance equivalent develops maximum field at the antennae. Thus, signal is trapped within the device and travels from the transmit antenna into the receive antenna with proper impedance matching, as shown in Figure 2.1.

\subsubsection{Mode Selection}

A circular waveguide was chosen for its low characteristic attenuation, comparative to alternate geometries; and for simplicity in machining.[16, 36] Circular waveguides also create mode cutoff isolation; overlapping TE and TM mode cutoff values are possible 

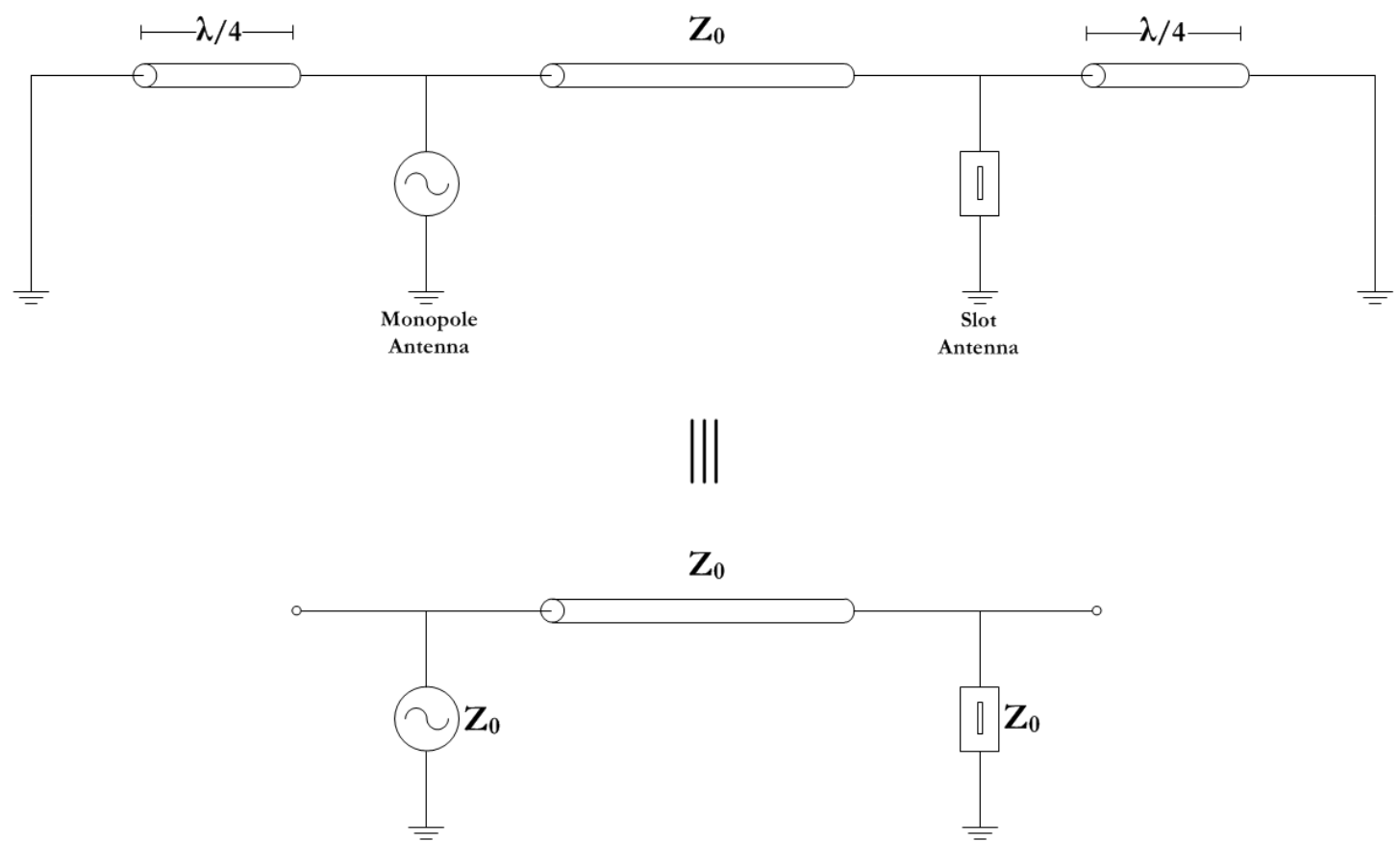

Figure 2.1: Traditional quarter-wave high field waveguide layout and equivalent circuit. The purpose of the circuit is to create optimal coupling and directivity between transmit and receive ports.

with rectangular waveguides.[41] Lower order modes within waveguides carry the smallest characteristic loss tangents, and the lowest possible mode within a circular waveguide is the $\mathrm{TE}_{11} \cdot[41]$

An induced potential across the void area of the ACC slot antenna was desired in order to generate current around the antenna, shown in Figure 2.2. Increased current density within the transmission area creates energy penetration into the quasi-particle and cooper-pair populations, which disrupts superconductivity. This required an electric field component within the waveguide parallel to the slot antenna surface and orthogonal to the slot width dimension. 

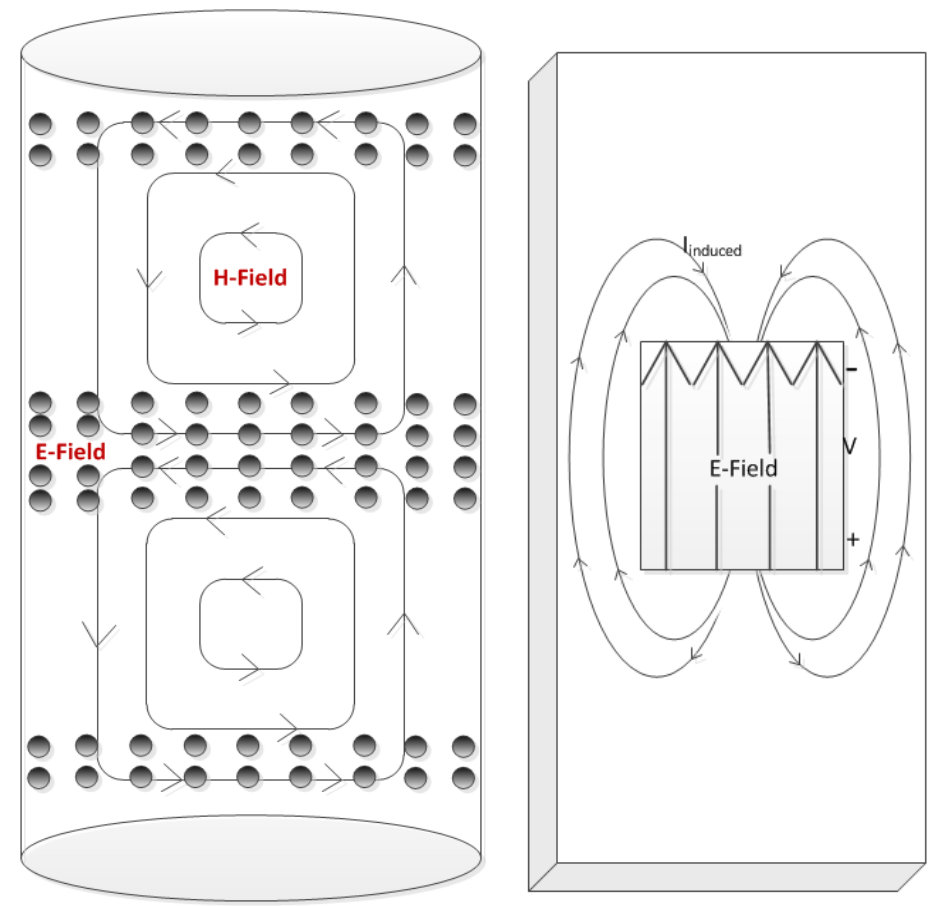

Figure 2.2: Induced current within $\mathrm{Nb}$ due to high E-field orientation. The left waveguide image illustrates circular longitudinal $\mathrm{H}$-field components, with an $\mathrm{H}$-null and lateral E-maximum in the center of the structure. Subsequent ACC chip coupling effects are shown on the right.

For a transverse electric waveguide, the magnetic field component is oriented longitudinally (z-axis shown on Figure 2.3); with a lateral electric component across the diameter of the cylinder ( $\mathrm{x}$-axis in Figure 2.3). The $\mathrm{TE}_{11}$ mode develops two longitudinally circulating magnetic fields, which cancel in the z-axis center, maximizing the lateral electric field in the null $\mathrm{H}$-field node. Thus, a $\mathrm{TE}_{11}$ mode would maximize coupling in the null $\mathrm{H}$-field longitudinal center of the waveguide.

Higher order evanescent modes were assumed to be cut off, absent within the waveguide structure. These modes, however, may develop reactive contributions to the device at substrate boundaries and electrical excitation points.7 [41]

\footnotetext{
${ }^{7}$ Tested devices showed significant evanescent mode effects at frequencies below the cutoff, as discussed in Chapter 6.
} 


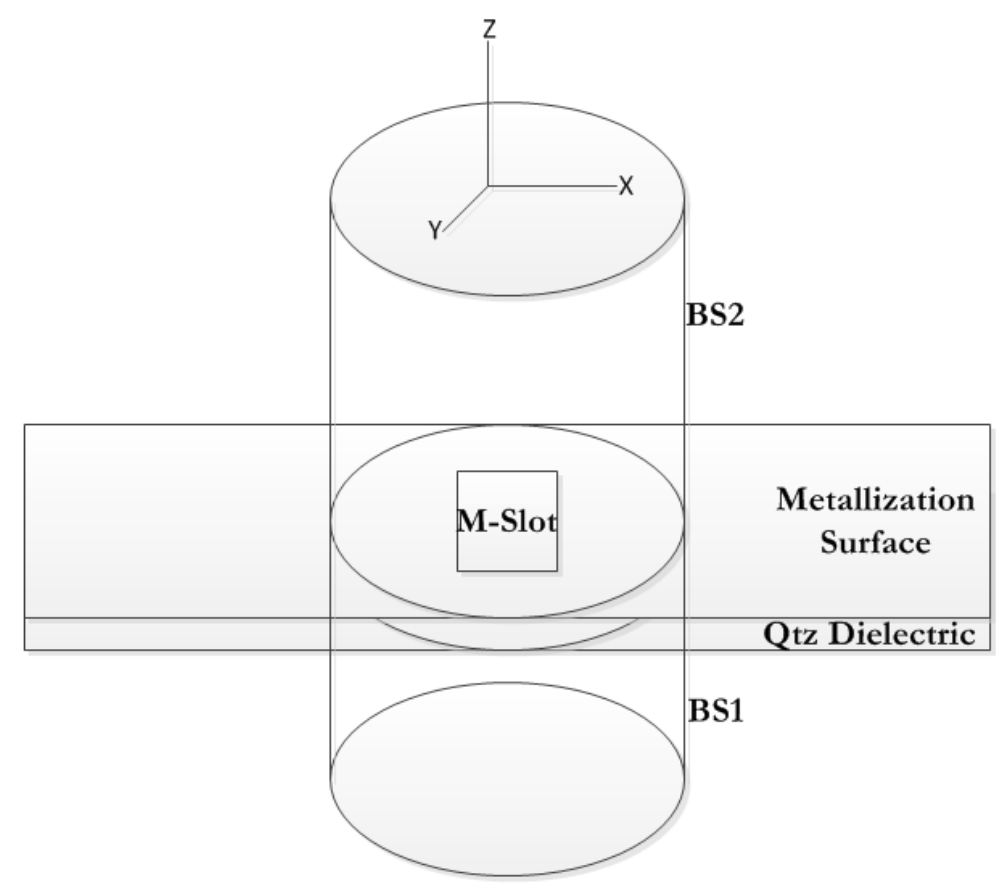

Figure 2.3: Illustrative layout of the waveguide geometry with major design parameters identified.

\subsubsection{Geometry Design}

Waveguide geometry design, based on desired signal propagation, involves solving the Helmholtz wave equation for the longitudinal field within a geometry.[41] For a circular TE waveguide, the longitudinal $\mathrm{H}$-field is defined in cylindrical coordinates as follows.

$$
\begin{gathered}
H_{z}(\rho, \phi, z)=h_{z}(\rho, \phi) e^{-j \beta z} \\
\left(\frac{\partial^{2}}{\partial \rho^{2}}+\frac{1}{\rho} \frac{\partial}{\partial \rho}+\frac{1}{\rho^{2}} \frac{\partial}{\partial \phi^{2}}+k_{c}^{2}\right) h_{z}(\rho, \phi)=0
\end{gathered}
$$

A wave number is the spatial equivalent of a given frequency. The cutoff wave number, $k_{c}$ is defined per mode by solving the Helmholtz representation in terms of Bessel's differential equation with the function of the first kind, $J_{n}(x)$.[41] The derivative of the Bessel function 
is used to define the radial electric field, which must be zero at $\rho=a$.[41] Here, the variable $a$ is the waveguide radius. This equates the fact that an electric field cannot exist at the metallic wall of a waveguide. It then emerges that the forcing condition for this to be true is a function of the wave number and radius.

$$
J_{n}^{\prime}\left(k_{c} a\right)=0
$$

A series of roots, $p_{n m}^{\prime}$, from the forcing function define the circumference $n$ and radial $m$ variations within the $x y$ plane, as shown in Figure 2.3, where a specific wave exists.[41] The combination of these variances defines a mode, $\mathrm{TE}_{\mathrm{nm}}$. Modal cutoff wave numbers are then found with the following relationship.

$$
k_{c, n m}=\frac{p_{n m}^{\prime}}{a}
$$

The $p_{n m}^{\prime}$ solution with the lowest value defines the dominant mode ( $\mathrm{TE}_{11}$ for a cylindrical geometry).[41] Propagation within the waveguide is defined by the intrinsic wave number $k$ of a given frequency through a substrate and that of the waveguide enclosing an identical substrate. Inferred from this relationship is the propagation frequency dependence on the waveguide radius. The propagation constant is defined as,

$$
\beta_{n m}=\sqrt{k^{2}-\left(\frac{p_{n m}^{\prime}}{a}\right)}
$$

where,

$$
k=\omega \sqrt{\mu \epsilon}
$$


and gives the effective propagation of a frequency within a given waveguide mode. The propagation constant must be a real number for a frequency to be supported.[41] Equation 2.5 assumes a homogeneous effective dielectric. Waveguides with mixed dielectrics at boundary surfaces must use a mixed dielectric model in calculations for $\beta$. This model was not applied to the ACC design because the quartz layer was situated at the transmission distance between the antennae, and as such, did not participate in the waveguide function. The propagation constant also changes the effective wavelength within the structure.[41] A guided wavelength is defined as,

$$
\lambda_{g}=\frac{2 \pi}{\beta}
$$

which becomes important when defining the longitudinal quarter-wavelength dimensions of the waveguide.

The second mode in a circular waveguide is the $\mathrm{TM}_{01}$.[41] Geometry design was based on a radius value which allowed propagation between the $\mathrm{TE}_{11}$ and $\mathrm{TM}_{01}$ cutoff points. These points carried $p_{n m}^{\prime}$ values of 1.841 and 2.405 respectively.[41] 


\subsubsection{Frequency Selection}

Considerations for the circular waveguide in $\mathrm{TE}_{11}$ mode were employed at a target operating frequency in close range of $20 \mathrm{GHz}$. The energy required to disturb the quasi-particle population without breaking cooper-pairs was defined by a two-energy gap model of multiband (small s-band and dominant cooper-pair breaking d-band) superconductivity in $\mathrm{Nb}$ [22]

$$
\Delta_{s}<\hbar \omega<\Delta_{d}
$$

where,

$\hbar=$ Planck's constant $^{\prime}$

$\Delta=$ Gap energy

Experiments by [22] indicate that $\Delta_{s}$ is on the order of $0.1 \Delta_{d}$. Using a conservative absolute zero approximation of $\Delta_{d}(0) \sim 3.14 \mathrm{meV}$, equation 2.8 gives a quasi-particle disruption frequency bandwidth of approximately $12 G H z-120 G H z$.[37, 49] This calculation neglects energy gap alterations from temperature and applied current, which serve to reduce the effective lower- and upper- bound quasi-particle disruption frequency.[49] Reasoning for this simplification rests on the fact that the device operating frequency was very close to the lower bound frequency, so would remain effective within a suppressed energy bandwidth.

Accounting for a $20 G H z$ maximum signal generator supply limitation, the $\mathrm{TM}_{01}$ cutoff of the waveguide was set at approximately $23 G H z$. This specification dictated the radius of the waveguide, and resulted in a $\mathrm{TE}_{11}$ propagation range of $17.58 \mathrm{GHz}-20.00 \mathrm{GHz}$. 
Table 2.1: Initial mode property specifications at $20 G H z$, assuming vacuum propagation parameters $\epsilon_{0}$ and $\mu_{0} . \mathrm{TM}_{01}$ propagation constant $(\beta)$ and guided wavelength $\left(\lambda_{g}\right)$ are imaginary values, indicating the mode is cut off at this frequency.

\begin{tabular}{ccccccc}
\hline \hline & $\begin{array}{c}\mathrm{f}_{\mathrm{C}} \\
(\mathrm{GHz})\end{array}$ & $\begin{array}{c}\omega_{\mathrm{C}} \\
\left(\mathrm{s}^{-1}\right)\end{array}$ & $\begin{array}{c}\mathrm{k}_{\mathrm{C}} \\
\left(\mathrm{mm}^{-1}\right)\end{array}$ & $\begin{array}{c}\text { Radius } \\
(\mathrm{mm})\end{array}$ & $\begin{array}{c}\beta \\
\left(\mathrm{mm}^{-1}\right)\end{array}$ & $\begin{array}{c}\lambda_{g} \\
(\mathrm{~mm})\end{array}$ \\
\hline $\mathrm{TM}_{01}$ & 22.97 & $1.735 \times 10^{11}$ & 0.481 & \multirow{2}{*}{5.0} & $0.236 \mathrm{i}$ & $-26.6 \mathrm{i}$ \\
$\mathrm{TE}_{11}$ & 17.58 & $1.104 \times 10^{11}$ & 0.368 & & 0.200 & 31.4 \\
\hline
\end{tabular}

Waveguide properties were ascribed as shown in Table 2.1. For an operating frequency of $20 \mathrm{GHz}$, the guided quarter-wavelength was $7.85 \mathrm{~mm}$. In compliance with machining needs, BS2 was specified at $6.35 \mathrm{~mm}$. BS1 contained the monopole transmit antenna. It was tuned with antenna placement coordinates, through parametric sweeps in HFSS, for optimal transmission length and field support. A BS1 length of $3.89 \mathrm{~mm}$ was given from optimizations, which equaled $\sim 0.12 \lambda_{g}$ at the optimally tuned frequency of $19.8 \mathrm{GHz}$.

\subsection{Chip Design}

The design of the ACC chip required specification of the physical support for the superconducting $\mathrm{Nb}$ transmission strip-line, as well as matallization layers for desired transmission constraints.

\subsubsection{Dielectric}

The ACC metalized thin film layers were supported on a quartz dielectric. Wafer specification was governed by requirements of dielectric constant and material thickness. Dielectrics within a circular waveguide can become resonant if their material dielectric constant is high ${ }^{8}$

\footnotetext{
${ }^{8}$ Resonance effects were also discovered to exist when antenna dimensions favored $\lambda_{Q t z}$ for a given frequency. Notably, $\lambda_{Q t z}$ at $4 G H z$ is nearly identical to $\lambda_{g}$ at $20 G H z$ within a vacuum.
} 
$\left(10 \leqslant \epsilon_{r} \leqslant 100\right)$. [41] The dielectric constant of amorphous quartz at room temperature is $\sim 3.8$, and at $4.2 K$ is approximated at $\sim 4.5$, which is below the resonance threshold. However, crystalline quartz carries a dielectric constant of 4.5, which increases in cryogenic conditions. Calculations were based on an approximated value $\mathrm{n}$ the range, 3.8-4.5. Dependence of the frequency level on dielectric constant was also unaccounted in the initial design.

Wafer thickness was specified as $\ll \lambda_{g(Q t z)} / 2$, to prevent resonance ${ }^{9}$ and dielectric impedance effects at frequencies near $20 G H z$. This served to create a slight capacitance; rather than a significant alteration to the transmission impedance, which would result from a thickness on the order of the wavelength. The guided $20 G H z$ wavelength within a quartz dielectric at $4.2 \mathrm{~K}$ was approximated to be $14.0 \mathrm{~mm}$ using an altered propagation constant,

$$
\beta=\sqrt{\epsilon k_{0}^{2}-(3.832 / a)^{2}}
$$

where 3.832 is the $p_{n m}^{\prime}$ solution for the dominant circular dielectric resonator mode. This gave a dielectric width requirement below the range of $0.7 \mathrm{~mm}$. Because the quartz chip was not trimmed to the waveguide radius, a second width threshold calculation was done for the case where the dielectric did not functionally adopt the waveguide diameter. In this case, an unguided $20 \mathrm{GHz}$ wavelength through amorphous quartz was estimated as $7.1 \mathrm{~mm}$, with an acceptable thickness in the range of $0.35 \mathrm{~mm}$. Two wafer thicknesses were employed for chip fabrication, $0.350 \mathrm{~mm}$ (crystalline) and $0.515 \mathrm{~mm}$ (amorphous).

\footnotetext{
${ }^{9}$ Longitudinal dimension of a dielectric resonator is $\lambda_{g(Q t z)} / 2$.
} 


\subsubsection{Antennae}

The waveguide was excited through a monopole antenna, which was created from a coaxial RF cable. This was chosen for ease of construction and adjustment. The outer grounded sheathing on the cable was trimmed to expose a transmit length, which was tuned in HFSS simulations. The trimmed quarter-wavelength monopole dimension was specified as $7.85 \mathrm{~mm}$, and used as the initialize value in HFSS parameter sweeps. A final dimension of $3.14 \mathrm{~mm}$ for the monopole was found, through simulation, to provide the lowest S11 return loss to the monopole ${ }^{10}$

Experiments by [1] and [32] indicate optimized monopole antenna miniaturization at $0.095 \lambda$, which is the electrical length of the $3.14 \mathrm{~mm}$ monopole at the HFSS design frequency of $19.8 \mathrm{GHz}$. Characteristic impedance of a quarter-wave monopole $(36+i 20 \Omega)$, normalized to $50 \Omega$ on a Smith chart, is $0.095 \lambda$ from the short-circuit end of the chart. HFSS optimizations resulted in a miniaturized antenna, based on a $50 \Omega$ characteristic coaxial line impedance, to create a similar electrical length from the short-circuit edge of the waveguide where the antenna was inserted.

HFSS tuning also provided the ideal transmission path; lowest reflection was attained through placing the monopole directly beneath the quartz, with minimal vacuum space between. With this juxtaposition, the signal would travel between antennae through a single medium and avoid reflection boundaries at quartz-ain ${ }^{11}$ interfaces. Impedance of the slot

\footnotetext{
${ }^{10}$ Extension within the waveguide was lengthened during testing, while still exposing the same trimmed surface of $3.14 \mathrm{~mm}$. This heightened coupling at low frequencies.

11 "Air" is used for simplicity of discussion. The waveguide actually contained helium in mixed liquid-vapor states, with complex and temperature-dependent phase development. Because the dielectric properties of helium within these states are in very close range of air within a vacuum $(0.1-4.0 \%$ deviation $)$, the simplified
} 
antenna was based on Babinet's principal and Booker's relationship, as well as the ratio of effective aperture area through quartz ${ }^{12}[3,41]$

$$
Z_{\text {slot }}=\frac{\eta^{2}}{4 Z_{T}}\left(\frac{\lambda_{g(Q t z)}^{2}}{\lambda_{g}^{2}}\right)
$$

where,

$\eta=$ Impedance of free space $\approx 377 \Omega$

$Z_{T}=$ Transmit antenna radiation impedance

Based on its dual quarter-wave radiant monopole, the slot impedance was $\approx 149-i 83 \Omega$.

The $\lambda_{g} / 4$ wavelength slot dimensions were adjusted to account for transmission effects within a waveguide and through the dielectric. The width dimension served to provide adequate current density constriction regions, which were loosely defined during $\mathrm{Nb}$ film characterization $\sqrt{13}$ Length initialization was set at $7.85 \mathrm{~mm}$, matching $\lambda_{g} / 4$ at the initialize frequency of $20 G H z$, and then adjusted with parametric sweeps in HFSS. The final dimensions for the modified receive slot were optimized at a length of $7.26 \mathrm{~mm}$ and width of $5.88 \mathrm{~mm} \cdot{ }^{14}$ The receive antenna coupling dimension was reduced below $\lambda_{g} / 4$. This was a direct cause of patterning on a dielectric substrate, which causes antenna miniaturization as well as bandwidth widening. [8]

model was retained.[29]

${ }^{12}$ Assuming no loss and unity gain. This was allowable because the effective area ratio for an impedance adjustment depends solely on factors of $\lambda^{2}$.

${ }^{13}$ This also led to a broadband antenna design because the width defines allowable frequency bandwidth in a slot antenna.

${ }^{14}$ Broadband dimension. 


\subsubsection{Metallization}

Two layers of metallization were employed to create the ACC chip: niobium and gold (Au). $\mathrm{Nb}$ was meant as the superconducting strip-line and the Au was used to secure the interface between $\mathrm{Au}-$ plated four-point probes and the $\mathrm{Nb}$ surface.

\subsubsection{Nb Transmission Substrate}

Niobium layer thickness was specified to attain an ACC critical current of approximately $2 A$ at the transmission width of $W=5.92 \mathrm{~mm}$ around the modified slot antenna. Niobium carries an approximate critical current density $\left(J_{C}\right)$ of $2 M \mathrm{~A} / \mathrm{cm}^{2}$. The critical current is defined through multiplying $J_{C}$ by the cross-sectional superconducting transmission area $(A=d W) .[15$, 49] Using this relationship, the necessary film thickness $(d)$ was calculated,

$$
d=\frac{I_{C}}{J_{C} W}
$$

which gave an approximate film thickness of $20 \mathrm{~nm}$. Thin film $\mathrm{Nb}$ carries higher impedance than thick film. A $20 \mathrm{~nm}$ thick deposition layer of $\mathrm{Nb}$ was approximated to carry a normalstate resistance of $\sim 10 \Omega / s q$ at cryogenic temperatures. The sheet impedance of the film affects the antenna function when coupling RF into the superconducting fluid. To optimize coupling, the sheet impedance was varied in close range of $10 \Omega / s q$ during simulation; and assessed with $>90 \%$ absorption at a sheet impedance of $8 \Omega / s q .[15]$ Allowing for the slightly reduced impedance, a film thickness of $30 \mathrm{~nm}$ was designated ${ }^{15}$

\footnotetext{
${ }^{15}$ Scale-up for industrial grade devices would require higher amperage allowance at comparable sheet impedance, necessitating use of a superconducting medium other than $\mathrm{Nb}$.
} 


\subsubsection{Au Contact Pads}

During preliminary testing on a beta-version ACC chip, four-point probes with Au plating contacted the bare $\mathrm{Nb}$ surface. This gave rise to a galvanic reaction between the dissimilar metals when current was applied from the outer probes to the chip. These reactions burned holes through the $\mathrm{Nb}$ layer at the probe contact points. Subsequently, Au pads were designed as a top layer on the $\mathrm{Nb}$ strip to prevent testing failures from galvanic effects and $\mathrm{Nb}$ oxidation at point contacts. They provided an $\mathrm{Au}-\mathrm{Au}$ contact for the probes, without significantly modifying the device function because the current flow favored the superconducting substrate.

\subsection{HFSS Simulation}

Original design initializations were tuned in HFSS. The tuned results were then analyzed to interpret the function of the simulated design. Sensitivity analysis was applied to key parameters for predicting variance introduced by small error margins in fabrication testing set-up.

\subsubsection{Waveguide Function}

BS1 and the receive antenna through the quartz dielectric acted as parallel transmission paths, fed from the sending port at the coaxial cable. The addition of BS2 behind the receive antenna created a circuit that resembled stub impedance matching. [41] At the optimized

\footnotetext{
${ }^{16}$ However, characteristics of Andreev reflection at this boundary were noticed when the device was superconducting.
} 
frequency of $19.8 \mathrm{GHz}$, the effective transmission path through the quartz created a reduction in receive slot aperture area of $\sim 1 / 5$. A miniaturized antenna at $0.095 \lambda_{g}$ allowed directivity of a quarter-wave monopole, matched to the $50 \Omega$ coax at a reduced radiation impedance.

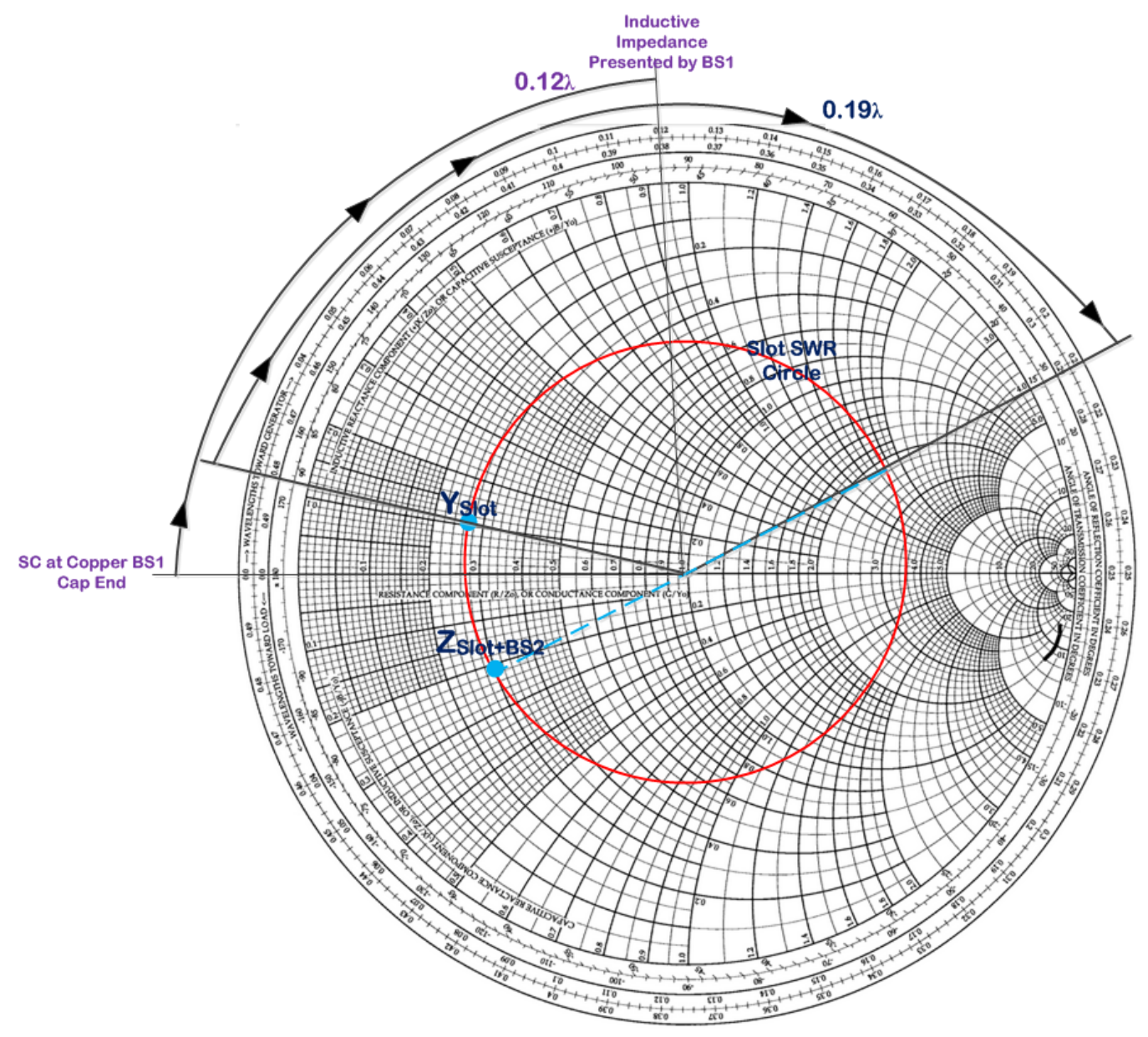

Figure 2.4: Smith chart tracking of the functional quality provided by tuning stubs, BS1 and BS2. Rather than quarter-wave high-field caps, they were optimized in HFSS for impedance matching between the antennae. 
Applying a proportional reduction to the transmit radiation impedance of the monopole from the quarter-wave value gave $13.7+i 7.6 \Omega$. Normalizing the receive antenna impedance value to a characteristic impedance of $50 \Omega$, and then converting to an admittance on the Smith chart, allowed assessment of the BS2 shunt stub matching contribution. From the admittance value, a length of $0.19 \lambda$ was plotted in the clockwise direction along the SWR circle. The adjusted admittance was then converted back to a final effective impedance of $13.5-i 12 \Omega$, showing a close conjugate match to the transmit antenna. This process is detailed in Figure 2.4.

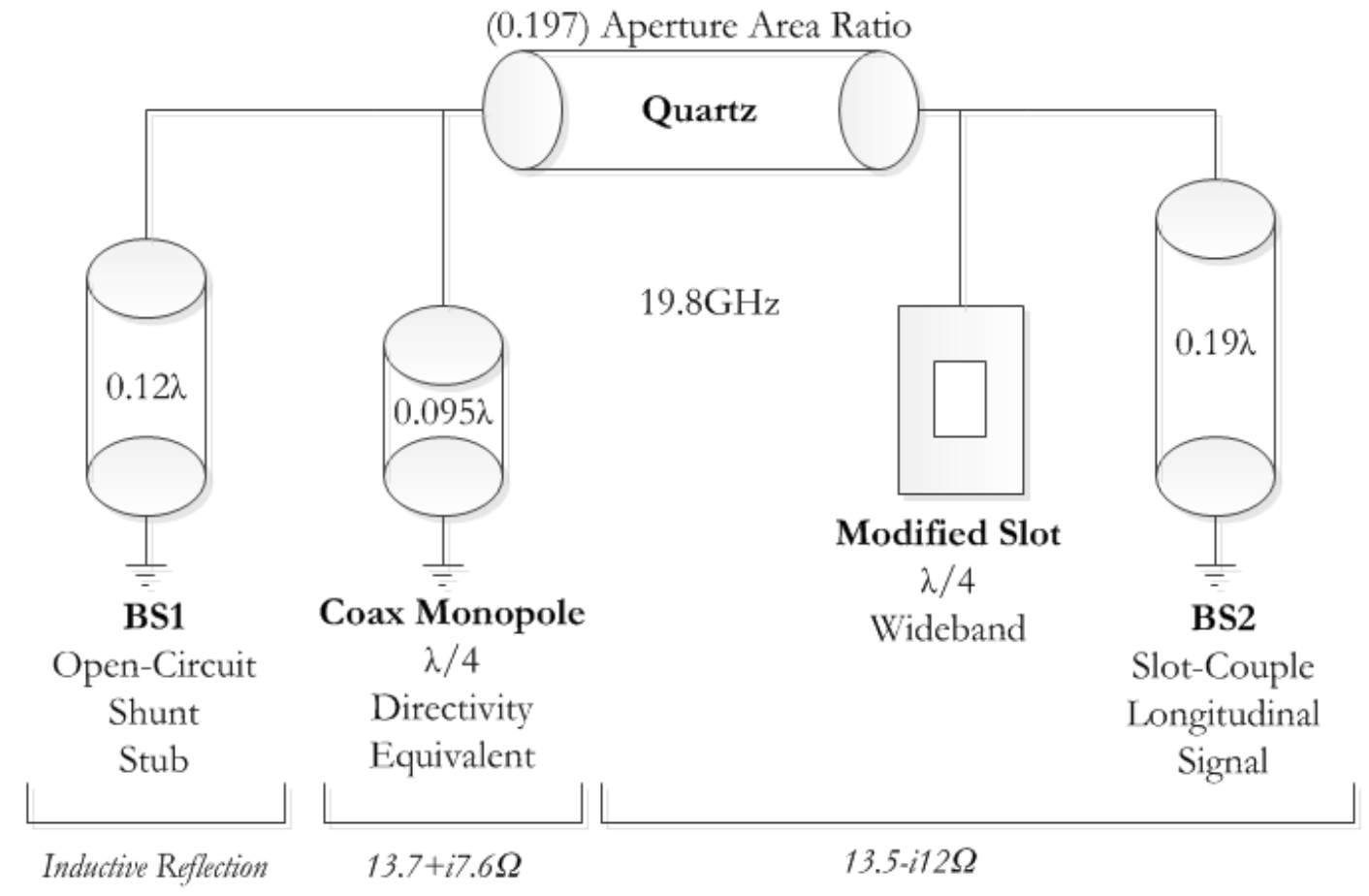

Figure 2.5: Equivalent circuit schematic and functional values at the optimized design frequency, for quasiparticle redistribution, of $19.8 \mathrm{GHz}$. 
BS1 presented a shunt path with an electrical distance $\sim \lambda / 8$, which was terminated in a grounded short circuit at the bottom surface of the waveguide. The electrical distance of BS1, when exposed to the transmit antenna, appeared highly inductive and affected an open circuit at high frequency. Thus, BS1 directed signal toward the receive terminal. The combined resistance of the coax and BS1, presented to any reflections back from the receive terminal, was found via the Smith chart to be $26.8+i 41.5 \Omega$. Together, these functional qualities formed directivity toward the slot. The equivalent circuit is shown in Figure 2.5.

\subsubsection{Parametric Sweeps and Sensitivity Analysis}

Parameter sweeps were executed in HFSS for minimal S11 results, with the monopole antenna identified as port 1 . Variations were made with regard to $\mathrm{Nb}$ sheet impedance, quartz chip width, exact frequency specifications, antenna and BS1 tuning, as well as current density assessment. 


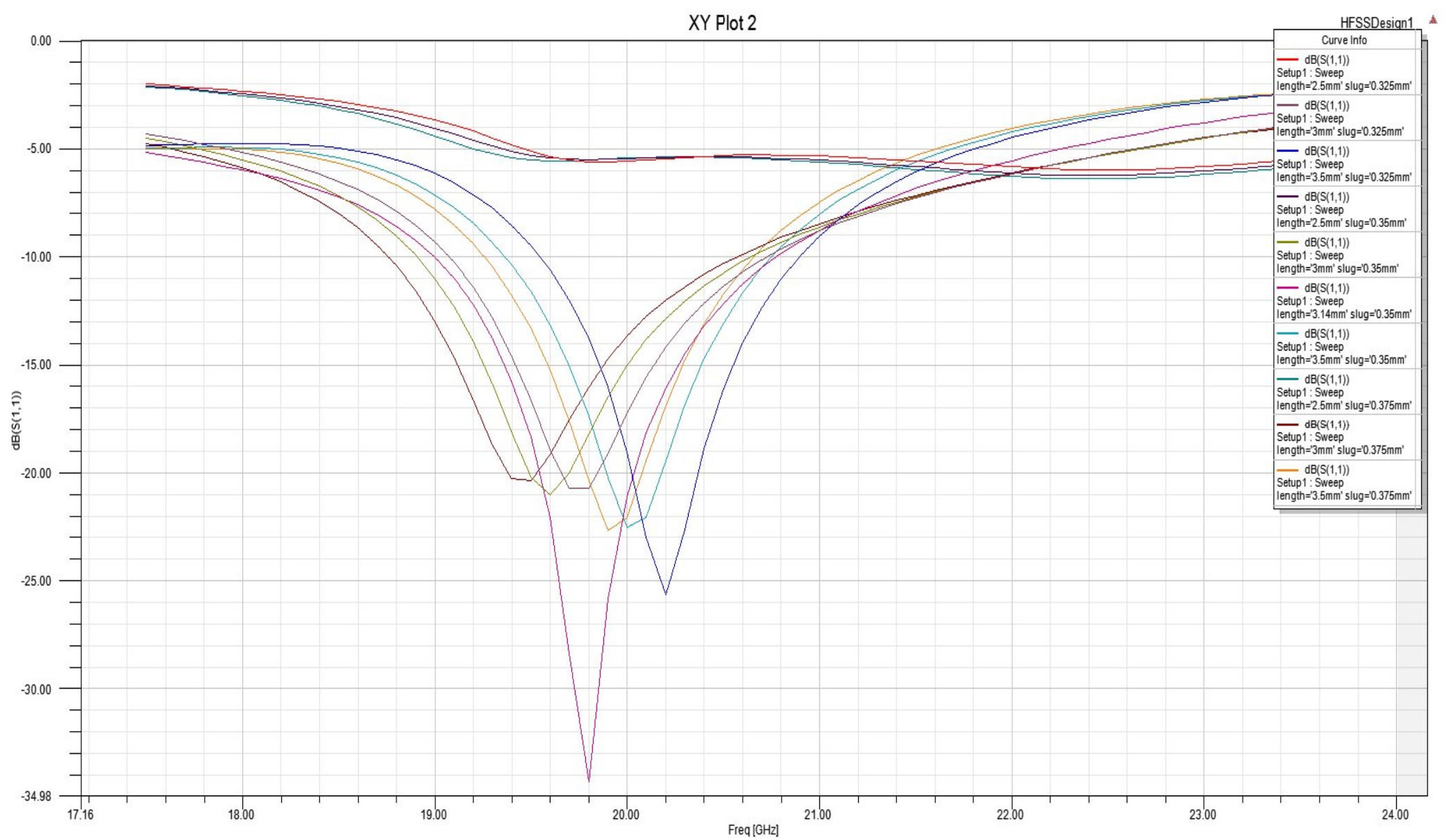

Figure 2.6: Parametric analysis, sweeping monopole antenna length. Minimal S11 occurred with parameter values: $B S 1=3.89 \mathrm{~mm}, B S 2=$ $6.35 \mathrm{~mm}(0.25 ")$, monopole length $=3.14 \mathrm{~mm}$, slot length $=3.63 \mathrm{~mm}$, slotwidth $=2.94 \mathrm{~mm}$, and $Q t z_{\text {slug }}=0.35 \mathrm{~mm}$. These parameters correspond with a frequency of $19.8 \mathrm{GHz}$. 
Figure 2.6 shows the optimized design, with parameter values listed in the caption. This gave the aforementioned impedance matching circuit for 19.8GHz. Apparatus design was based on this assessment. The simulations show variance in the monopole transmit length. Though an exact fabricated length of $3.14 \mathrm{~mm}$ was necessary to acquire an extremely low S11 value, comparable length variations returned acceptable S11 values $(<-20 d B)$. This confirmed that the length of the transmit monopole antenna was not vitally sensitive to small errors in fabrication. Further variance in physical ACC parameters was investigated to ensure success of the device with potential alterations in the quartz thickness and $\mathrm{Nb}$ metallization layer. 


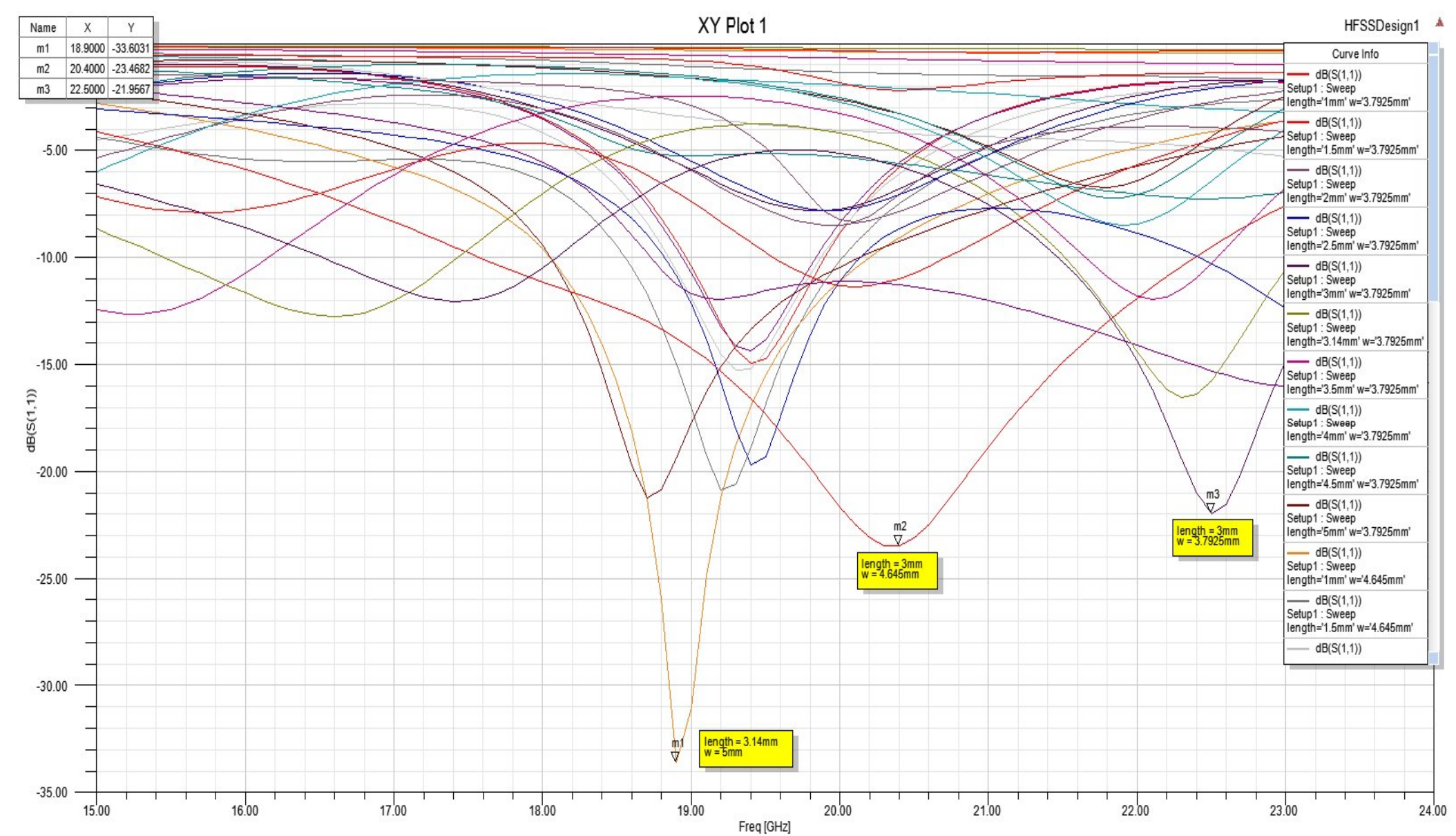

Figure 2.7: Parametric monopole transmit sweeps, with the quartz dielectric adjusted to a thickness of $0.515 \mathrm{~mm}$. The originally specified $3.14 \mathrm{~mm}$ extension maintained minimal $\mathrm{S} 11$, but the optimal frequency was reduced to $18.75 \mathrm{GHz}$. 
Figure 2.7 shows parameter sweeps, replacing the $0.350 \mathrm{~mm}$ quartz with a thicker $0.515 \mathrm{~mm}$ sample. The original dimensions remained as the optimized values, with an approximate $1 G H z$ reduction in the optimized coupling frequency. This showed that both quartz samples would function in the allowed frequency range of the waveguide, with all other design parameters held constant. However, tests run on the $0.515 \mathrm{~mm}$ quartz showed a slightly higher sensitivity to monopole extension length variance. 


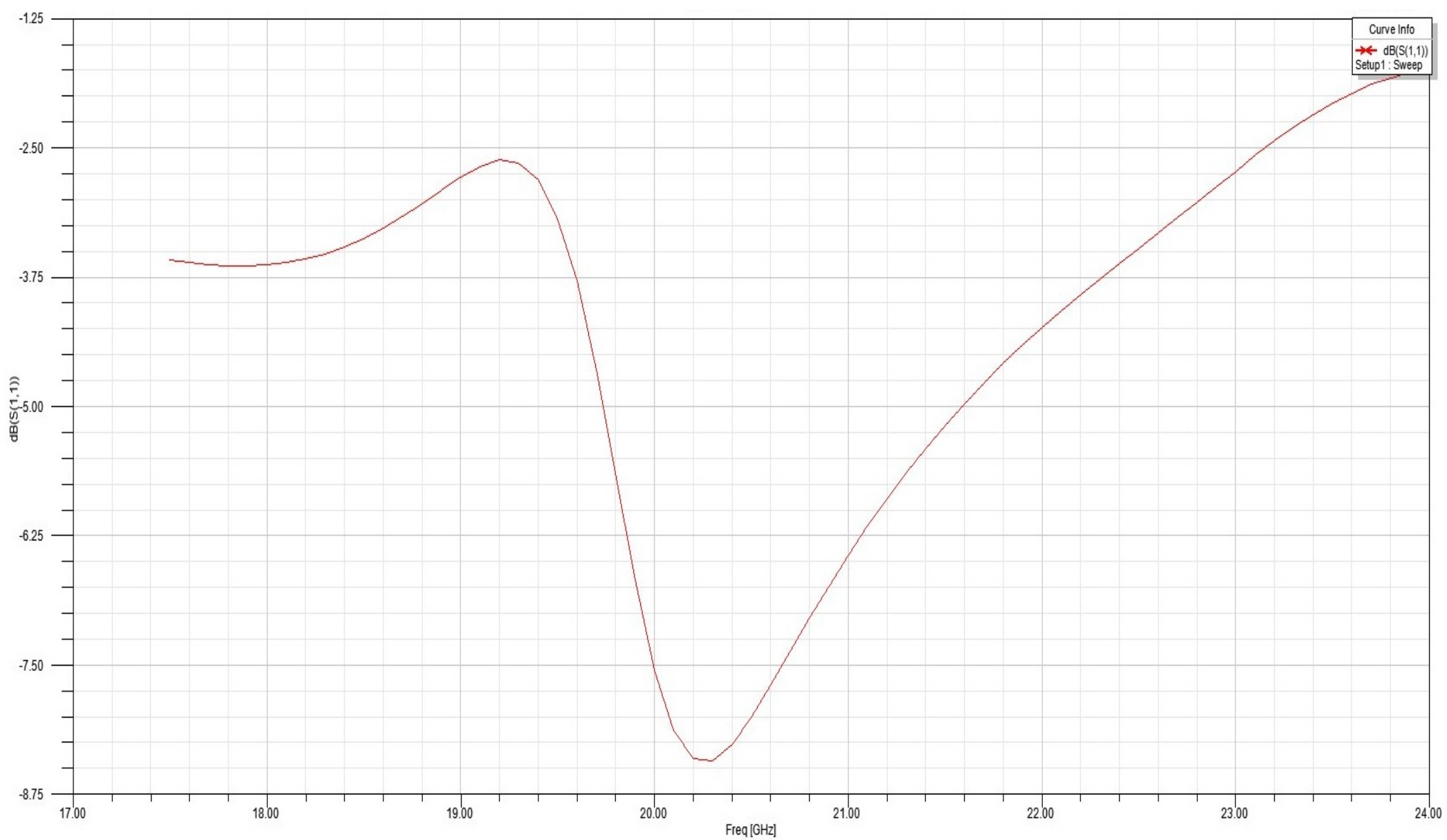

Figure 2.8: S11 analysis incorporating extremely low sheet resistance $\left(S R=8 e^{-6} \Omega /\right.$ square $)$ of the $\mathrm{Nb}$ sample, with all other design variables held constant. The S11 characteristic within the waveguide frequency range showed varied sensitivity to extremely low sheet resistance. 
NIOBIUM SHEET RESISTANCE VS. DBS(1,1)

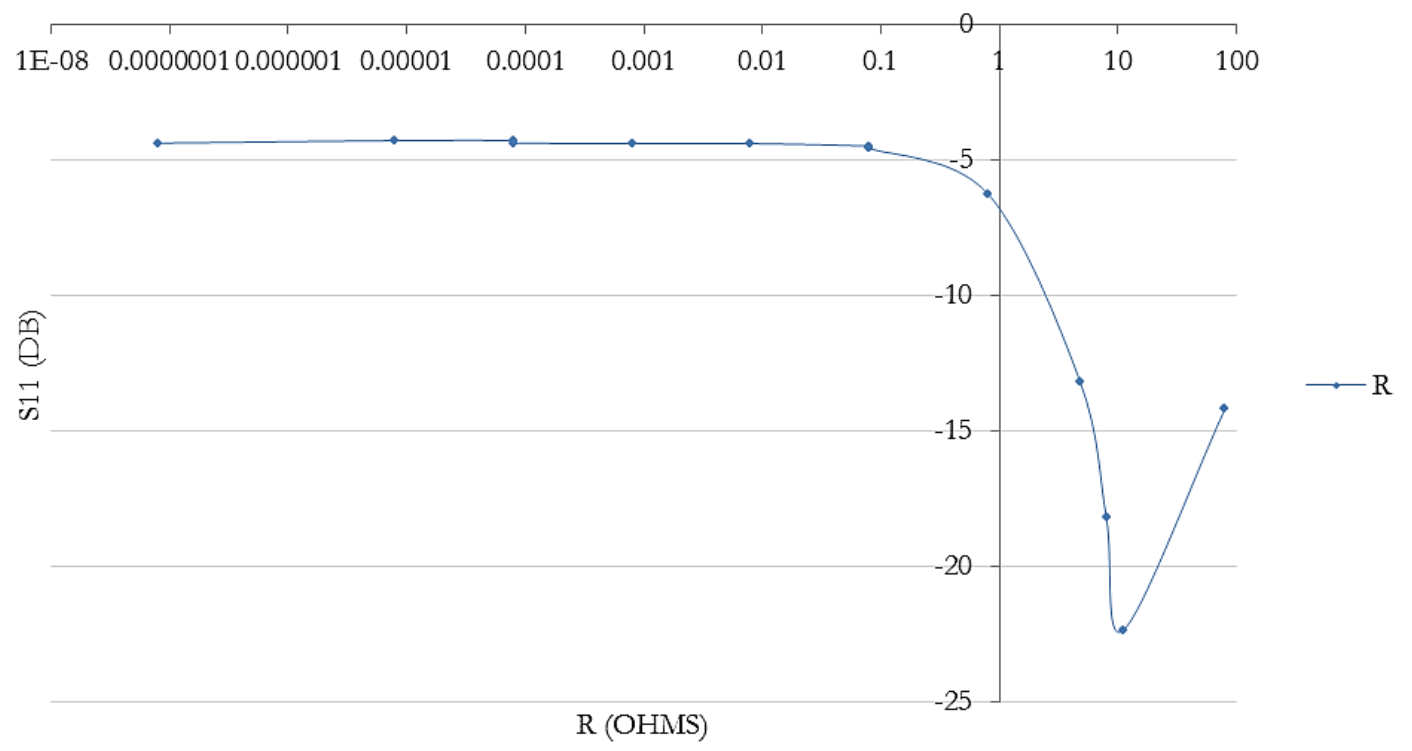

Figure 2.9: Swept $\mathrm{Nb}$ sheet resistance data at $19.8 \mathrm{GHz}$ showed sufficient robustness against large error margins, with a maximum $\mathrm{S} 11$ at $-4.8 d B$. The $\mathrm{x}$-axis is shown in log scale to account for swept orders of magnitude in the variable.

The final major sensitivity analysis was conducted on the sheet resistance of the $\mathrm{Nb}$ sample. Normal-state cryogenic $\mathrm{Nb}$ substrate sheet impedance was approximated as $8+$ $i 0.08 \Omega$. Any potential error in the approximation was accounted through wide-range impedance sweeps. Figure 2.8 shows an S11 profile for extremely low impedance, which exhibits acceptable $(<-3 d B)$ S11 only in the range near $20 G H z$. Figure 2.9 illustrates a full resistance sweep of simulated S11 values at $19.8 \mathrm{GHz}$, confirming that coupling was not critically sensitive to the $\mathrm{Nb}$ sheet impedance approximation within close range of $20 \mathrm{GHz}$. 


\subsubsection{HFSS Animation Stills}

Figures 2.10 and 2.11 show HFSS CAD schematic animation stills of the preliminary design at maximal points for the longitudinally travelling electric wave and induced surface current density on the $\mathrm{Nb}$ strip, respectively. They reinforce the conclusion that the waveguide would support a longitudinally travelling electric wave, with directivity from the monopole send antenna toward the slot receive antenna.

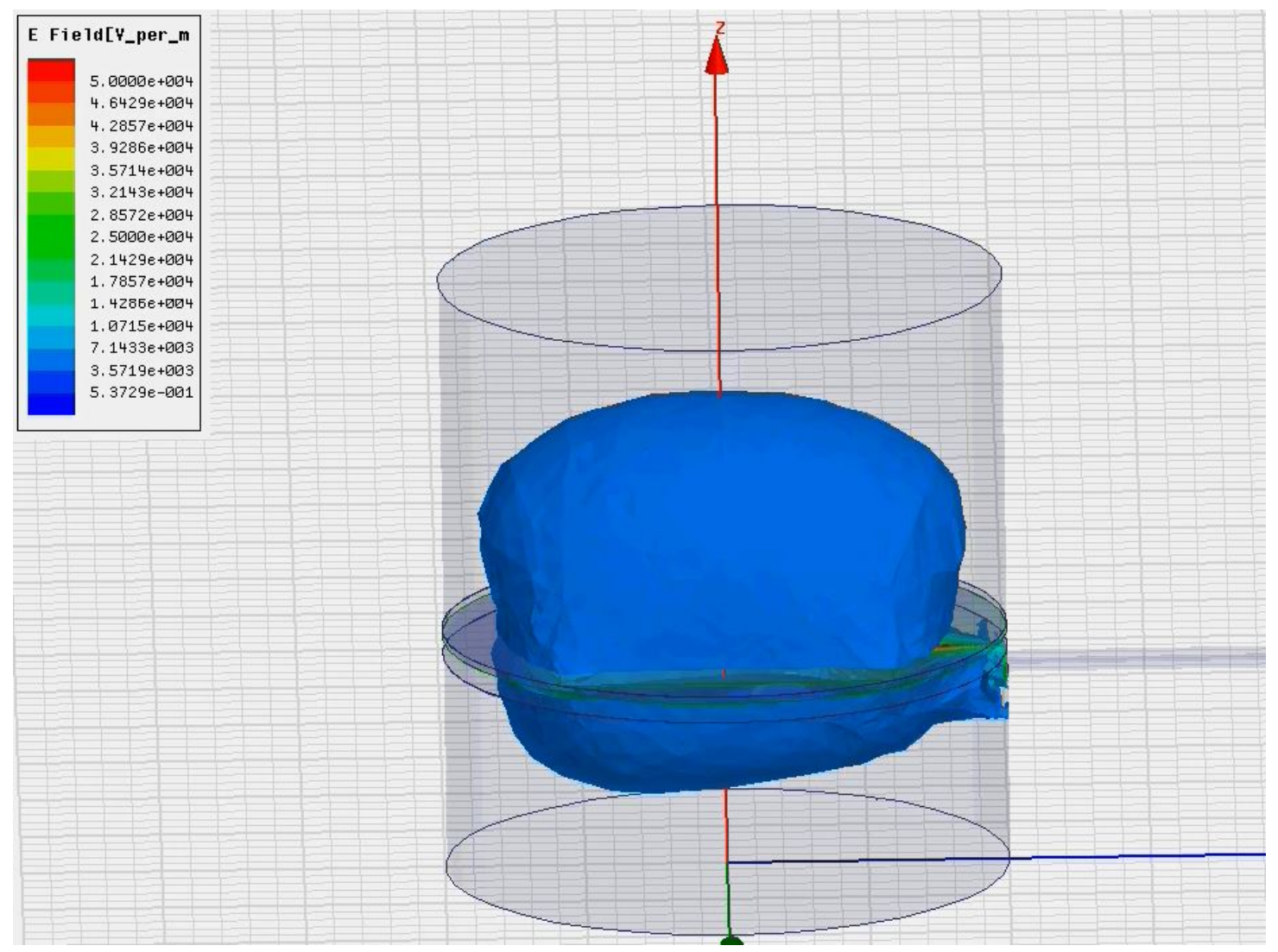

Figure 2.10: E-field animation at $19.8 \mathrm{GHz}$ showed a large longitudinally travelling electric wave. It carried an intensity of $\sim 2 \mathrm{~V} / \mathrm{mm}$, which was consistent with design specification.

Figure 2.10 confirmed the predicted inductive function of BS1, as the electric wave did not enter into BS1 beyond an evanescent attenuation threshold. Thus, directivity of the wave at the design frequency was toward the slot antenna. 


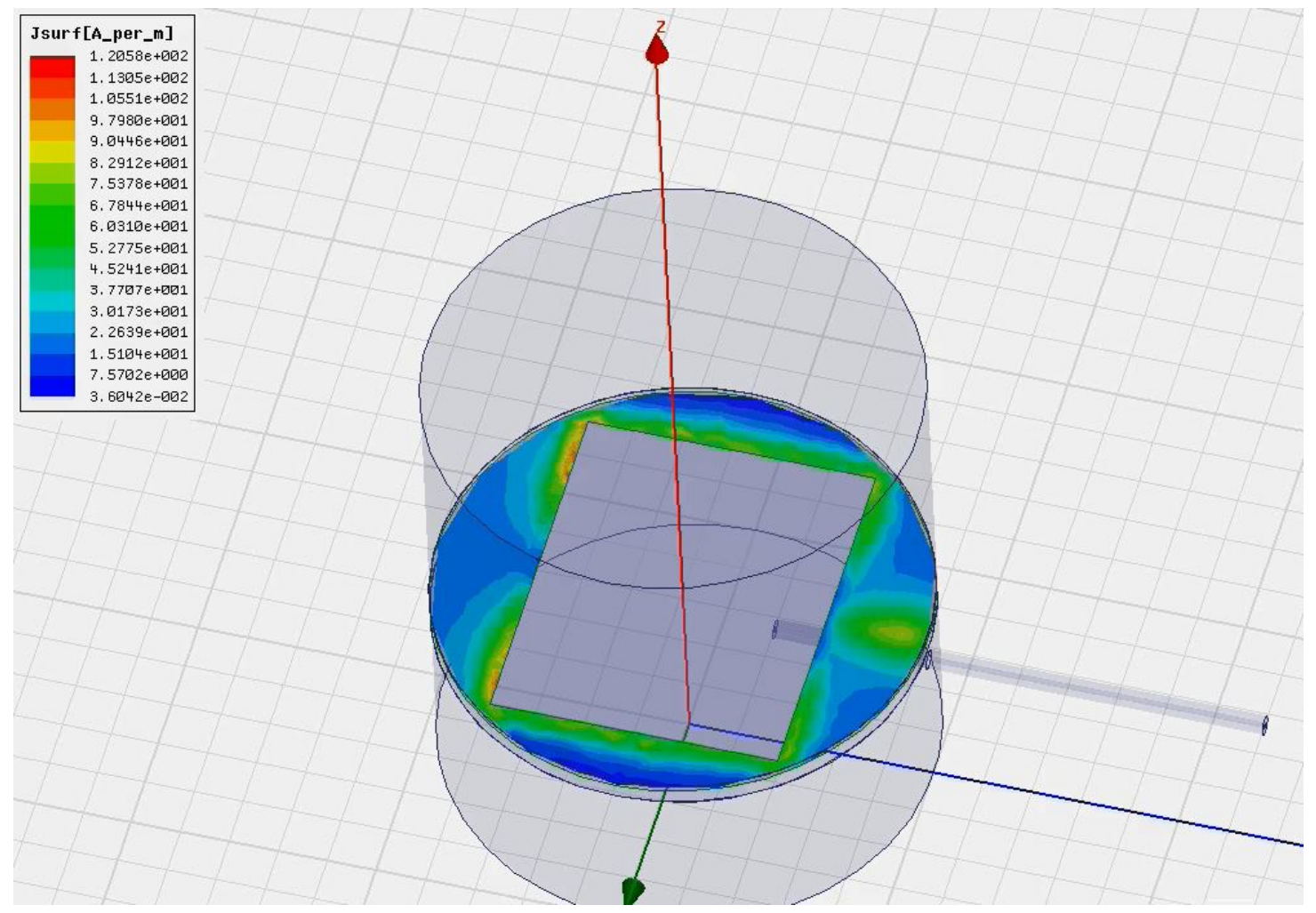

Figure 2.11: Induced surface current density profile at $19.8 \mathrm{GHz}$ indicated a strong induced energy perturbation from RF coupling, with $\sim 20 \mathrm{~mA} / \mathrm{mm}$.

Figure 2.11 illustrates proper coupling into the slot receive antenna, giving an additive energy perturbation within an order of magnitude of the critical current. The relative magnitude indicated that the perturbation would be sufficiently strong to alter superfluid dynamics. 


\section{Chapter 3: Fabrication}

Device fabrication focused solely on the ACC chip. The waveguide was machined by Aric Datesman and the majority of the testing apparatus was created by Aaron Datesman.

Chip fabrication included deposition and patterning of $30 \mathrm{~nm} \mathrm{Nb}$ and $50 \mathrm{~nm}$ Au layers on a quartz substrate. Two sample substrates were used; a $0.350 \mathrm{~mm}$ thick single-crystal $\mathrm{X} \& \mathrm{Y} \& \mathrm{Z}$ orientation quartz wafer, and a $0.515 \mathrm{~mm}$ thick amorphous quartz wafer. The $0.350 \mathrm{~mm}$ thick quartz wafer was excessively brittle for standard cleanroom procedures, and required mounting on a sacrificial silicon $(\mathrm{Si})$ carrier wafer.

The metal deposition process was planned to take place in the PSU Center for Electron Microscopy and Nanofabrication (CEMN). Due to CEMN equipment failures, the chips were instead sent to a collaborator, Arthur Lichtenberger, at the University of Virginia (UVa) for sputtering. Wafer dicing was also outsourced to UVa because PSU PNNA cleanroom facilities were not yet equipped with wafer dicing machinery. Au lithography and $\mathrm{Nb}$ reactive ion etching (RIE) were performed on the samples using the PSU PNNA cleanroom facility. 


\subsection{Sample Mounting on Si Carrier}

Black mounting wax, with a characteristically high melting temperature, was used. The employed wax was specified as high strength, very hard, with a melting point of $100^{\circ} \mathrm{C}$ and solubility in acetone. This wax was chosen primarily for its high melting point, due to the need for baking mounted chips during photolithography stages. Typically, mounting is performed with a specific mounting block, placed on a heating surface.[24] This was replaced with a layer of aluminum foil on a hot plate in the PSU PNNA cleanroom.

The sacrificial silicon sample was placed on the aluminum-lined hotplate and baked for five minutes at a temperature setting of $115^{\circ} \mathrm{C}$. After the preliminary heating stage, a volume of approximately $290 \mathrm{~mm}^{3}$ was applied uniformly over the surface of the silicon wafer. The surface wax layer was heated for an additional three minutes until reaching an homogeneous liquid melting point. The $0.35 \mathrm{~mm}$ thick quartz wafer was then placed on the wax surface and gently pressed to full contact by hand; and then removed from the hot plate and cooled to room temperature. Conventionally, the initial heating process would be followed by clamping the mounted samples together before cooling.[24] This allows exactitude in uniformity of wax thickness, which was not a critical concern for this device because of relatively large feature sizes in the working surface geometries.

Dismounting the samples from the black wax connection includes heating to $\sim 150^{\circ} \mathrm{C}$, separating the two wafers, spraying samples with trichloroethylene (TCE) for initial wax thinning and degreasing, then spraying and soaking in acetone to remove the remaining film on the chip surface.[30] This was carried out at UVa after the chips were diced. 


\subsection{RCA Cleaning}

RCA cleaning was performed as a method to remove contaminants from sample surfaces prior to several processing steps. Modifications of the cleaning method were applied before deposition, photolithography and testing procedures. In addition to maintaining quality of the sample, RCA cleaning also protects process equipment from cross-contamination. RCA cleaning is a very common industrial process and is named for the Radio Corporation of America, where it was developed in 1965.[35] Its main application is the prevention of oxidation and contaminant diffusion on silicon in the semiconductor industry; but it is also useful in general surface preparation for processing. [35] The cleaning method comprises three sequential steps:

\section{Organic Clean:}

This removes organic matter like oils and dust from the sample surface, using strong alcohol-based solvent solutions.

\section{Oxide Removal:}

Surface oxidation of metals and substrates can prevent proper adhesion during deposition, as well as altering functional properties of the finished device. Performance deterioration is of specific concern in conductors because oxidation changes metallic bonds to ionic bonds, the latter of which cannot share valence electrons between atoms. This functionally changes oxidized metallic surfaces from conductors to insulators. Oxide removal is particularly important if the solution for the organic clean step contains a mix of water and acidic solvents, which can create a redox reaction on 
the sample surface. This step is accomplished through the use of corrosive acidic solutions, due to their effectiveness in attacking oxides.

\section{Ionic Clean:}

Ionic contaminants can be left on a surface in the form of acidic or metallic residues, which create a parasitic conductivity in solutions. This is harmful because it promotes corrosion, as well as surface augmentations from dendrite growth.[17, 45]

Often, samples are soaked in chemical cleaning solutions; however this creates a threat for re-absorption of impurities back into the sample surface. For this reason, organic cleaning of the ACC chip utilized Q-tip solvent application, followed by spraying of isopropyl (IPA) and methyl $(\mathrm{MeOH})$ alcohols with the sample mounted on a spin coater for quick drying. The oxide removal and ionic cleaning were done within a contained vacuum chamber, using Argonne ion bombardment, as part of the deposition process. Oxide removal was not critical for the quartz wafer substrate because it is characteristically an oxidized material. The ionic clean was important in preventing electrochemical reactions between the quartz surface and niobium layer. During the Au deposition it was essential to ensure minimal oxidation on the $\mathrm{Nb}$ layer because Au cannot bond effectively to a significantly oxidized surface. The sealed deposition vacuum chamber prevented oxidation of the $\mathrm{Nb}$ surface, alleviating the need for intermittent cleaning between layer depositions. Organic cleaning was the only RCA step performed on the ACC sample during photolithography and testing because oxidation and ionic contamination were not significant concerns in these environments. 


\subsection{Evaporation and Deposition Processes for CEMN Machines}

The original deposition recipe for use with the CEMN KJLC AXXIS system is outlined in Appendix A. It was assessed during an equipment training session.[4, 5] Because the deposition process was outsourced for this project, the science and theory of metal deposition was not included within the scope of this work.

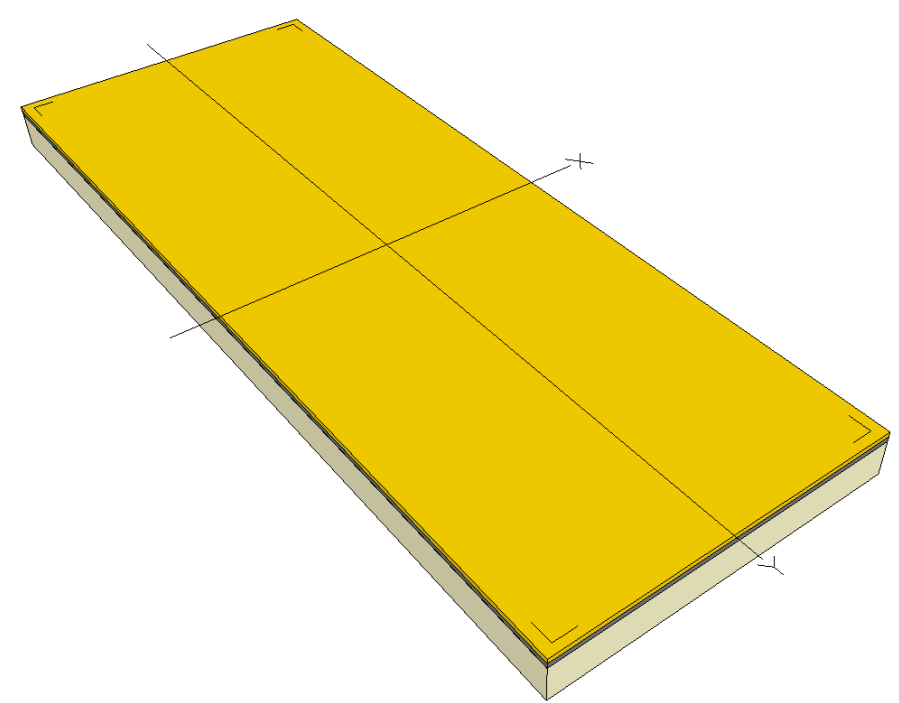

Figure 3.1: Illustrative representation of fully deposited ACC chip before patterning of the $50 \mathrm{~nm}$ Au and $30 \mathrm{~nm} \mathrm{Nb}$ layers. 


\subsection{Au Contact Pad Photolithography and Wet Etch}

The top surface Au layer was etched to create contact pads for Au probes in the testing apparatus. They were patterned using photolithography with a positive photoresist, then etched in an aqueous potassium-iodine-based Au etchant. Masking features for the photolithography stage are shown to-scale in Figure 3.2
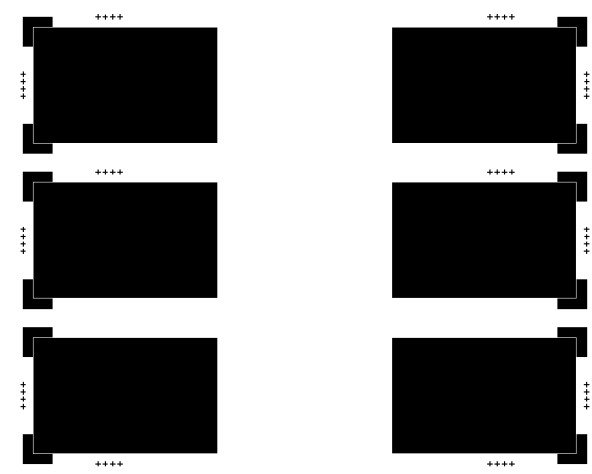

Figure 3.2: ACC01 Au Mask (shown to-scale), used for lithographic creation of probe contact pads.

\subsubsection{Photolithography}

Photolithography employs light-sensitive dye, called photoresist (PR), for transferring masked features to a substrate. The dye acts as a protective barrier, preventing etchants from reaching the substrate material below. Positive PR creates an absence of protective barrier after ultraviolet (UV) light exposure and development, while negative PR acts in the exact opposite manner.[4, 21] The PR is applied on a spin coater, with an angular velocity specified according to desired PR thickness. [4, 43] The PR layer must be sufficiently thick not to etch more rapidly than the substrate below it, in etchants that do not act exclusively on the substrate. Before light exposure, the PR must be soft-baked in order to create heightened 
photosensitivity, and also drive off the dye solvent. If the dye solvent remains in the PR, it will be less robust against etchants and provide poor substrate protection. [4, 21]

Light exposure was executed with a contact mask aligner. This method places the PR layer in direct physical contact with the patterned surface of the photo-mask, and focuses UV light through the mask onto uncovered surfaces of PR (where the mask is transparent). The wafer and mask are held together with a vacuum. In the case of a reflective under-layer, like $\mathrm{Au}$, it is important not to overexpose the masked wafer. This is because the Au can reflect light to PR surfaces beneath the edges of the masked features and create undercutting. [4] After light exposure to the positive PR on the ACC chip, it was developed in a sodium hydroxide $(\mathrm{NaOH})$ and water solution to dissolve the light-exposed PR geometries.[52] The remaining PR layer looked the same as the patterning mask. The patterned PR was then hard-baked to assist adhesion to the substrate and solidify the protective layer. [4, 21] This process was also used when creating a protective $\mathrm{PR}$ layer for $\mathrm{Nb}$ etching. 


\subsubsection{Au Etching}

Iodine bonds with Au to create gold iodide. Potassium is used to enhance solubility of the etchant.

$$
2 A u+I_{2} \rightarrow 2 A u I
$$

The addition of KI creates Lugol's Solution, which forms an anion of iodide that is readily soluble in water. An etchant solution then contains $\mathrm{I}_{2}, \mathrm{KI}$ and $\mathrm{H}_{2} \mathrm{O}$. The Au process algorithm is outlined in Appendix B, and the fabricated Au pads are illustrated in Figure 3.3.

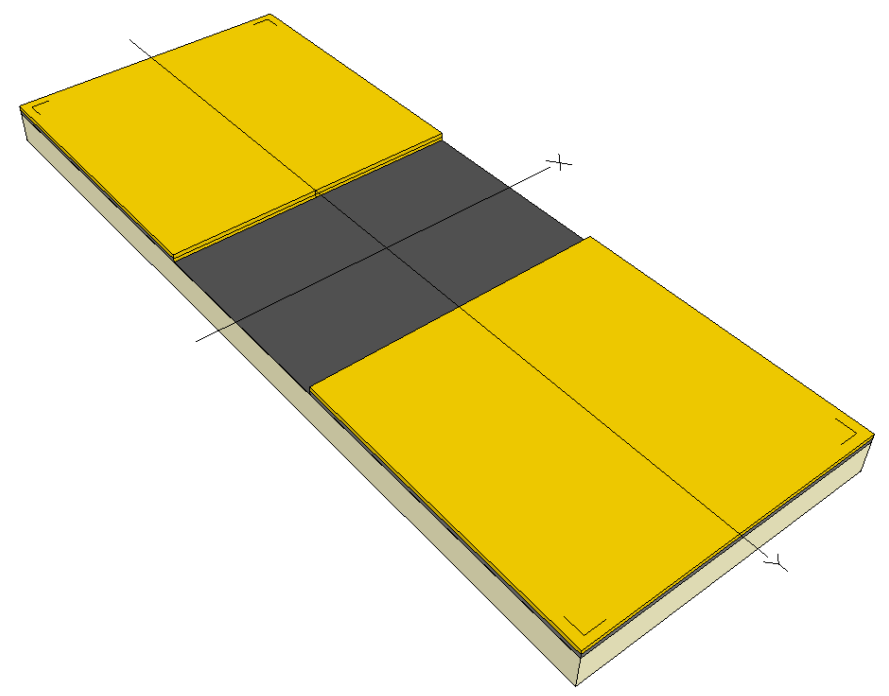

Figure 3.3: Illustrative representation of the patterned $50 \mathrm{~nm}$ Au layer after photolithography and etching. 


\subsection{Nb Etch Techniques}

Two $\mathrm{Nb}$ etching techniques were assessed, a wet etch and reactive ion etch, which are discussed in the following sections. Both techniques were employed; the RIE method showed favorable results in reaction control and safety. The RIE process is outlined in extensive detail, due to the complexity associated with recipe creation.

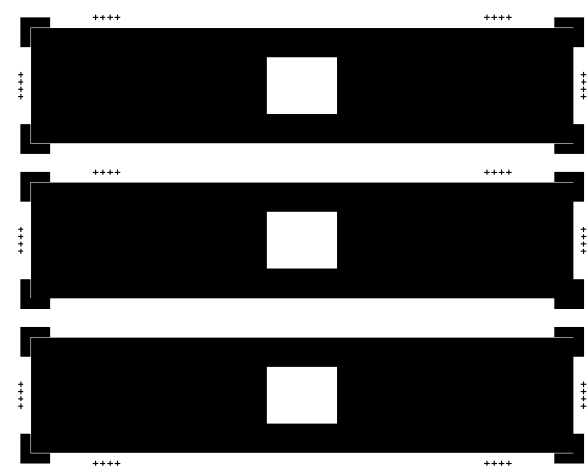

Figure 3.4: ACC02 Nb Mask (shown to-scale), used to create the base design patch receive antenna.

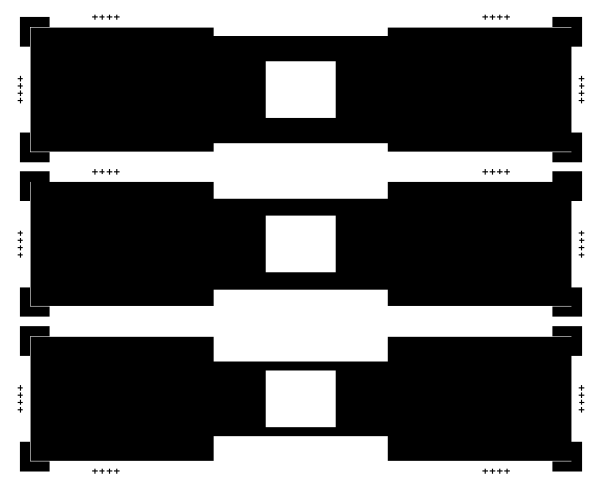

Figure 3.5: ACC03 Nb Trim Mask (shown to-scale), used to constrict current flow regions for tests with reduced $J_{C}$. 


\subsubsection{Wet Etch}

Common wet etch recipes for $\mathrm{Nb}$ combine hydrofluoric (HF) and nitric $\left(\mathrm{HNO}_{3}\right)$ acid; often prescribed in a 1:1 ratio, unless a third chemical is used for reaction curtailment and regulation. [9, 31, 39] The initial recipe, tested on an $\mathrm{Nb}$-deposited silicon chip prepared with a PR layer exposing etch-selected $\mathrm{Nb}$ substrate, adopted the 1:1 (HF: $\left.\mathrm{HNO}_{3}\right)$ ratio. It carried an expected etch time of 5 minutes.[39] The reaction formula, taken from [39], for this isotropic etch follows.

Individual Reactions,

$$
\begin{gathered}
6 \mathrm{Nb}+10 \mathrm{HNO}_{3} \rightarrow 3 \mathrm{Nb}_{2} \mathrm{O}_{5}+10 \mathrm{NO}+5 \mathrm{H}_{2} \mathrm{O} \\
\mathrm{Nb}_{2} \mathrm{O}_{5}+10 \mathrm{HF} \rightarrow 2 \mathrm{NbF}_{5}+5 \mathrm{H}_{2} \mathrm{O}
\end{gathered}
$$

Etching Reaction,

$$
3 \mathrm{Nb}+5 \mathrm{HNO}_{3}+15 \mathrm{HF} \rightarrow 3 \mathrm{NbF}_{5}+5 \mathrm{NO}+10 \mathrm{H}_{2} \mathrm{O}
$$

Equation 3.4 is a simple redox of the $\mathrm{Nb}$ metal. The $\mathrm{HNO}_{3}$ oxidizes $\mathrm{Nb}$ molecules, allowing subsequent reduction from the HF. This reaction can be violent and non-selective in the absence of a moderating chemical. It can also allow $\mathrm{Nb}$ to be redeposited onto the sample surface from debris associated with aqueous $\mathrm{NbF}_{5}$ near the chip surface. Due to the emergent volatile NO, the reaction is also caustic and produces a pungent yellow gas. 
A further complication associated with the $\mathrm{Nb}$ wet etch involves the safety hazards of HF. As a powerful and potentially fatal acid, HF requires handling with extreme care. Customized procedures, designed for this work, are outlined in Appendix C.

The initial mixture was composed of $100 \mathrm{~mL} \mathrm{HNO}_{3}(100 \%$ concentration) and $208 \mathrm{~mL}$ $\mathrm{HF}\left(48 \%\right.$ concentration) to achieve a $1: 1$ chemical ratio. When tested, a 1:1 (HF: $\left.\mathrm{HNO}_{3}\right)$ ratio was excessively violent and non-selective.

$\mathrm{The} \mathrm{Nb}$ etch rate was slower than the $\mathrm{Si}$ etch rate, due to unrestrained redox of the readily oxidized $\mathrm{Si}$. This caused a lack of reactive agent supply to the Nb surface. A similar issue arises with samples deposited on quartz, with HF reduction of the oxygen within the material lattice. In a deficient reactant mixture, the $\mathrm{Nb}$ etch lacked uniformity and developed varied metallization thickness across the etch-selected surface. The reaction progressed for 30s before the Si sample cracked in half at the point of contact with the plastic tweezers; and the portion left in the solution dissolved completely in $5 \mathrm{~s}$. This etch formula is better suited to samples deposited on substrates without redox potential. Otherwise, a buffer chemical is essential. Also, avoiding stagnation at the $\mathrm{HF}$ and $\mathrm{Nb}$ interface would require a higher concentration of oxidizing agent, to allow quick replenishment of reactive surface area. This would create a faster etch, preventing redeposition of $\mathrm{Nb}$ and allowing a lower degree of isotropy.

A second recipe was informed by the trial etch test and work performed by [31]. This work suggests various etch rates based on $\mathrm{HNO}_{3}$ concentration in solutions with $1 \%$ and $3 \% \mathrm{HF}$. A mixture of $55 \% \mathrm{HNO}_{3}$ concentration with a $3 \% \mathrm{HF}$ additive solute yielded an 
expected $7 \mathrm{~s} \mathrm{Nb}$ etch rate. Utilization of a $1 \% \mathrm{HF}$ additive solute would cause an approximate 33 s etch, which could pose issues in regard to substrate selectivity. The recipe for the new mixture included: $6.25 m L \mathrm{HF}(48 \%$ concentration $)+93.75 m L \mathrm{H}_{2} \mathrm{O}+185.72 m L \mathrm{HNO}_{3}$. This recipe was not tested due to time constraints and safety comparisons which favored reactive ion etching.

\subsubsection{Reactive Ion Etch}

Niobium was etched using RF capacitively-coupled reactive ion etching, which is a process of ionic exposure to remove deposited substances from wafer surfaces. Selected gasses are released within a metallic vacuum chamber in the presence of an oscillating electromagnetic field, which is applied to the bottom surface of the chamber through an RF source.[25] These conditions cause ionization of the gasses, creating plasma that reacts with the material surface and affects an etching process, which can be designed for anisotropy. [54]

The sequential progression of RIE chemical etching was defined by [54] as follows.

1. A reactive ion is created

2. Reactive ion encounters sample surface

3. Sample surface engages reactive ion through Van der Waals attraction

4. Covalent bond forms between the ion and sample surface

5. A new molecule forms on the sample surface

6. New molecule is volatile and detaches from sample surface as a gas

7. Product gas is extracted from the chamber through the vacuum pump 
The bottom chamber surface, called the platen or platter, holds the wafer and is electrically isolated from all other system components. It is connected to the RF power supply through a blocking capacitor, which isolates the platen from ground as well.[25, 54] RIE chambers are usually referred to as "parallel plate" because the top and bottom surfaces of the cylindrical structure act as an anode and cathode, respectively.[25]

Travelling within the plasma are both ions and free electrons, which carry different velocities because of vast deviations in their mass values. [4, 54] Ions are not subject to major motion fluctuations from the oscillating field because they have a much larger mass than electrons.[25] When the electrons hit the top grounded surface of the chamber, they exit the system to ground without causing disturbance. Because the platen is isolated from ground, its interaction with free electrons incites development of a negative self-bias DC voltage. [25, 54] The resulting bias is the mechanism establishing the platen as a cathode plate. It also creates a level at which the applied RF AC voltage is held, through superposition.[54] Polarization of the top and bottom plates in the cylindrical vacuum chamber creates a single direction of travel for the ions, toward the platen, thus making an anisotropic etch. This is the main advantage of RIE over chemical wet etching. Important factors governing etch rate and true anisotropy are: chamber pressure, applied RF power and frequency, gas composition and flow-rate, platen temperature and DC self-bias level.[30, 54] However, these factors are interconnected and also influence each other.[54] 
The Technics 800 Series Micro Reactive Ion Etch system that was employed for this etching process operates at an applied $\mathrm{RF}$ of $13.56 \mathrm{MHz}$ and has a manufacturer-recommended applied power level of $100 \mathrm{~W}$, which are common industry standards for RIE machines.[25] Reactive gasses used in this process were $\mathrm{CF}_{4}$ and $\mathrm{O}_{2}$. Ionization separates carbon from fluorine, allowing the positive fluorine ions to travel to the negatively charged platen and etch the $\mathrm{Nb}$ material surface by forming gaseous halides of $\mathrm{Nb} .[25,42$, 54] The ionic etching is mainly caused by chemical reactions. However, sputtering can also occur due to kinetic energy transfer from high-field collisions between the ion and the sample surface. [30, 54] Free carbon ions form polymer chains on the sample surface, which are removed by oxidation from $\mathrm{O}_{2}$ ions and high-power sputtering.[30] $\mathrm{CF}_{4}$ and $\mathrm{O}_{2}$ are pumped into the top portion of the chamber, and the resulting reactant compound gasses from the etched surface are pumped out as waste near the platen. These reactions attack both the exposed $\mathrm{Nb}$ surface and photoresist, meaning that the process must be engineered to create selectivity in etching the $\mathrm{Nb}$ at a faster rate than the photoresist.

Selectivity between $\mathrm{Nb}$ and photoresist etching depends heavily on the amount of reactive fluorine and $\mathrm{O}_{2}$ present in the chamber. The use of $\mathrm{O}_{2}$ increases both the $\mathrm{Nb}$ and photoresist etch rates, due to its interactions with the sample surface as well as the plasma particles. Increased chamber pressure, a function of gas flow-rate, causes better selectivity toward $\mathrm{Nb}$ [31, 44] Empirical tests on similar etches show a high Nb-to-photoresist selectivity (>15:1) with a $10 \% \mathrm{O}_{2}$ gas mixture at $300 \mathrm{mT}$ orr chamber pressure.[31] These tests were performed with $100 \mathrm{~W}$ input power and reported a platen self-bias DC voltage development 
Table 3.1: Nb RIE Recipe for $410 \mathrm{~cm}^{2}$ Platen at $21^{\circ} \mathrm{C}$

\begin{tabular}{lrl}
\hline \hline Pressure & 300 & mTorr \\
Power & 100 & $\mathrm{~W}$ \\
$\mathrm{CF}_{4}$ & 47 & $\mathrm{sccm}$ \\
$\mathrm{O}_{2}$ & 6 & $\mathrm{sccm}$ \\
Nb Etch Rate & 2.7 & $\mathrm{~nm} / \mathrm{min}$ \\
DC Bias & 81 & $\mathrm{~V}_{\mathrm{DC}}$ \\
Etch Time & 11 & $\mathrm{~min}$ \\
\hline
\end{tabular}

of $100 \mathrm{~V}$.[31] Reported values informed the initial ACC RIE recipe, as shown in Table 3.1 with slight system-specific modifications. Selectivity can be negatively influenced by excessively high cathode DC voltage. In which case, ions are accelerated toward the sample surface and etch through kinetic sputtering, vitiating chemisorption processes that allow selectivity.[54]

A simple equation to predict the DC bias voltage on the platen is developed in [54] and deals with the surface area ratio between the two electrodes in the chamber:

$$
\left|V_{D C}\right| \approx\left(A_{A} / A_{C}\right)^{n}
$$

where,

$V_{D C}=$ Voltage drop between plasma and cathode

$A_{A}=$ Anode area

$A_{C}=$ Cathode area

$n=$ Estimated etch factor 
The RIE chamber used in this work had a 3:1 anode:cathode ratio. The platen (cathode) had an area of $410 \mathrm{~cm}^{2}$, which is significantly smaller than the $730 \mathrm{~cm}^{2}$ cathode area that is commonly assumed in literature.[31, 44] Therefore, it was necessary to calculate the approximate bias $\mathrm{V}_{\mathrm{DC}}$, rather than assume the same value from empirical studies using electrodes of different dimensions.

The factor of $n$ in Equation 3.5 is based on assumptions of gas content, chamber pressure and power level during the etch. It is commonly characterized as either 1 or 4 , depending on etching conditions.[54] The $\mathrm{CF}_{4}$ gas releases fluorine, which is electronegative and tends to absorb free electrons; thus keeping them from the platen and lowering the DC voltage bias. $\mathrm{O}_{2}$ is used as a low-electronegative gas to repel electrons, which both raises the plasma pressure and creates more collisions. These collisions and repellent charges release electrons, and also force them to interact with the platen, thus raising the DC bias voltage level. It is important to balance the gas flow levels, in order to control the pressure in the chamber. Increasing the pressure too much will cause an excess of particles within the chamber, which reduces the distance travelled by an electron before encountering the platen. Functionally, this reduced distance lowers the energy that the electron carries to the platen and decreases the DC voltage bias magnitude. Based on the presence of $\mathrm{O}_{2}$, along with empirically assessed voltage behavior at the specified $300 \mathrm{mT}$ Torr gas pressure and $100 \mathrm{~W}$ power level, $n=4$ was assumed. The platen DC bias voltage was approximated at $81 \mathrm{~V}$. 
High chamber pressure and the interactions between multiple ionic species complicate the directionality in ionic flow toward the platen, which makes the etch more isotropic. [30] Adjustments to recover etch anisotropy can be accomplished through reduction of $\mathrm{O}_{2}$ and pressure levels, paired with increased power.[30] However, selectivity is lost with these adjustments because of interference from carbon polymers in the absence of $\mathrm{O}_{2}$; and also a greater sputtering likelihood from a denser population of more energetic electrons, resulting from increased power levels. Anisotropy is vital to etching when feature sizes are sufficiently small that $\sim 0.5 \mu \mathrm{m}$ of undercutting can change the functional shape of the device. [4, 30] Large feature sizes, on the scale of tens of $\mu m$, in the ACC meant that anisotropy could be sacrificed for selectivity. At a platen DC bias of $100 \mathrm{~V}$, there is sufficient energy to sputter platen metal onto the wafer surface.[30] This can impair the etch rate, create micro-masking and leave surface debris on the wafer.[4, 30] In prevention of these effects, the wafer was placed on a $2 " x 2 "$ ceramic stage on the platen. 
Platen temperature has a large effect on etch rate, and is especially significant in the case of undercutting that results from an over etch.[30] Typical RIE systems use an external water cooling mechanism to keep the platen at or below $21^{\circ} \mathrm{C}$. This temperature must be reduced when dealing with small feature sizes to keep energy for reactions at a minimum when all of the intended surface material has been etched. Lower temperatures require higher DC voltage on the platen to facilitate the $\mathrm{Nb}$ etch. Again, the ACC feature sizes dictated sufficient cooling at $21^{\circ} \mathrm{C}$. The $\mathrm{Nb}$ RIE process algorithm is outlined in Appendix $\mathrm{D}$

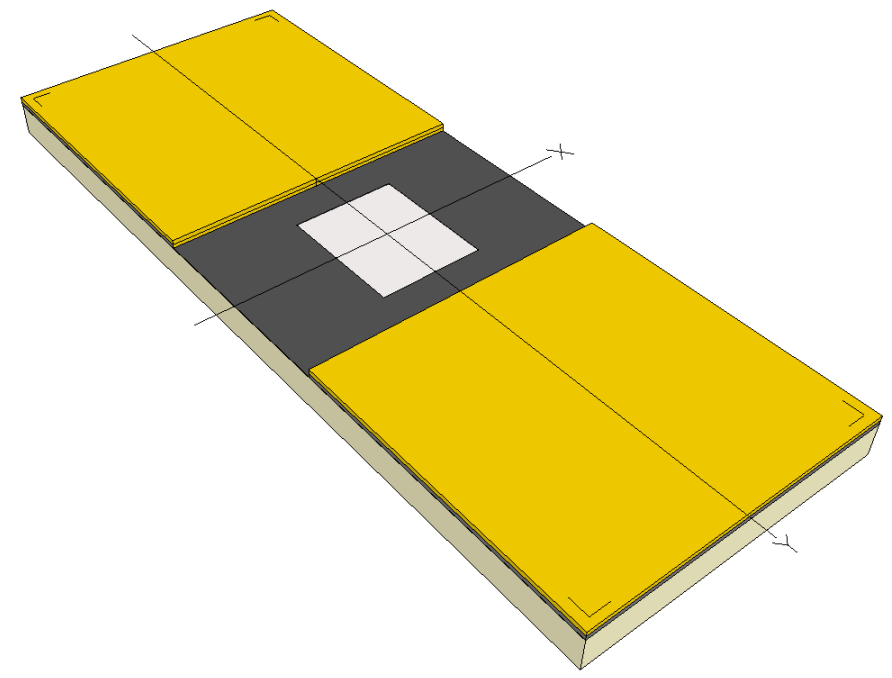

Figure 3.6: Illustrative representation of the fully patterned ACC chip after final RIE on the $30 \mathrm{~nm} \mathrm{Nb}$ layer. 


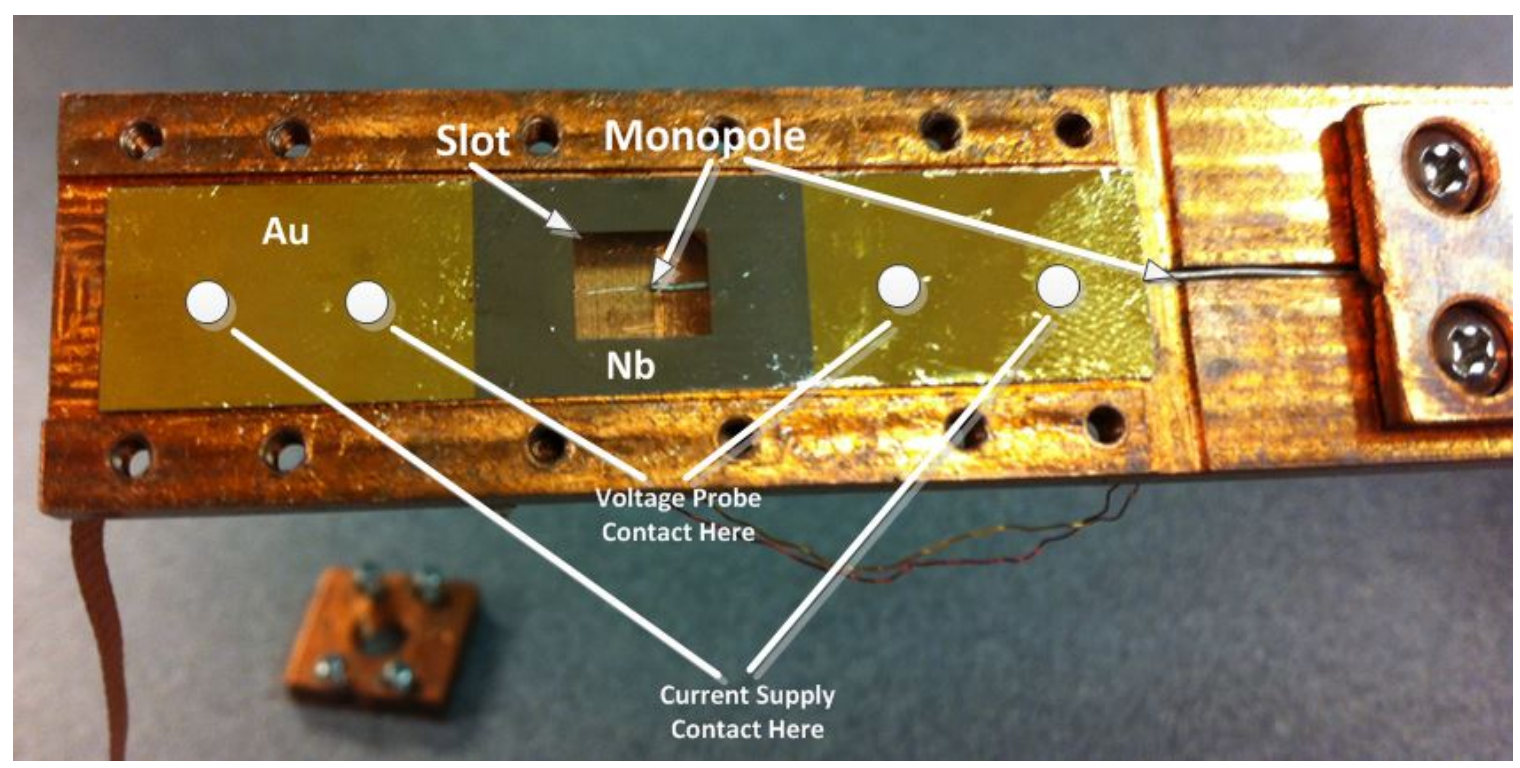

Figure 3.7: Fully fabricated ACC chip, shown in the copper holding stage of the device testing apparatus.

The final ACC chip, after completion of all fabrication steps, is shown within the device holding stage of the testing apparatus in Figure 3.7. The functional quality of each fabricated element is labeled, to define chip components within the context of the complete testing apparatus. The white four-point probe contact points are for illustrative purposes, and were not actually present on the chip surface. 


\section{Chapter 4: Experimental Methods}

\subsection{Apparatus}

The testing apparatus was built to function like a dipstick, which was positioned within a dewar containing liquid helium (LHe) under one atmosphere of pressure. The ACC was connected to the end of the dipstick, and thus submerged in a LHe bath near $4.2 \mathrm{~K}$. Temperatures under test were varied up to $T_{d}^{17}$ through adjusting the vertical position of the dipstick in relation to the liquid level within the dewar.

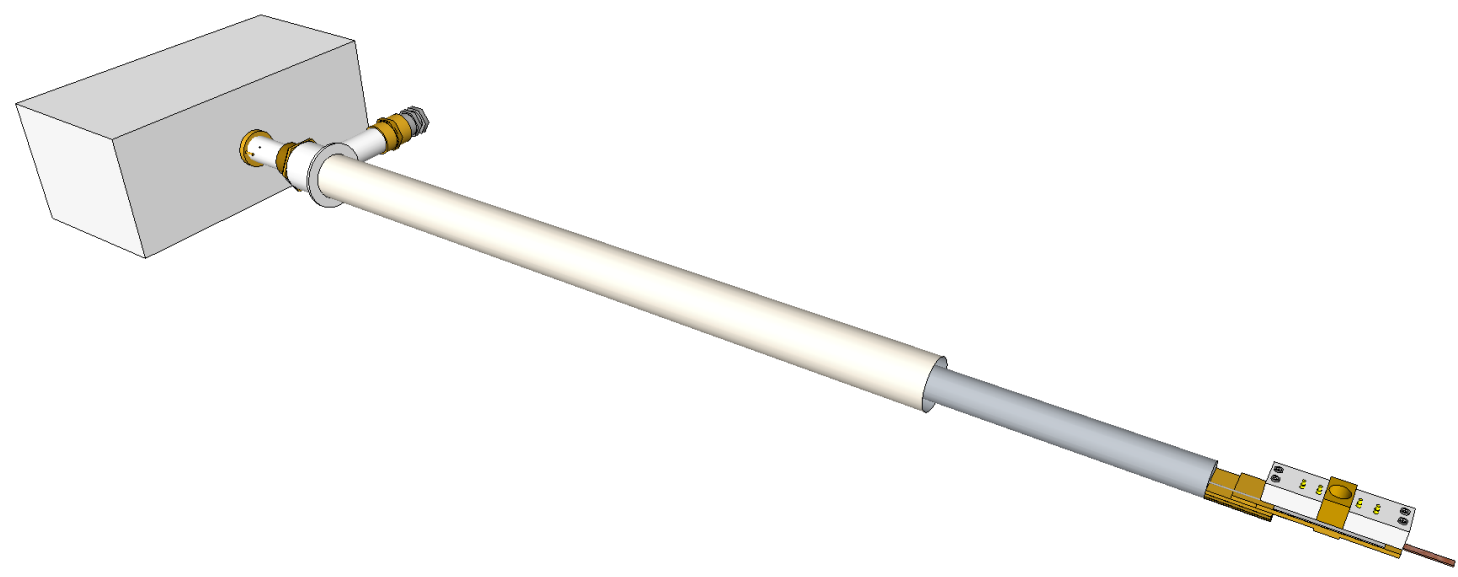

Figure 4.1: Illustrative model of dipstick testing apparatus with all major components visible.

\footnotetext{
${ }^{17}$ The critical temperature where a superconducting state will undergo transition to normal conductivity.
} 


\subsubsection{Dipstick}

Figure 4.1 gives a visual depiction of the dipstick apparatus configuration. Signal junctions between sources, meters and the ACC chip were contained within an aluminum cast electronic instrument case for EMI/RFI shielding. Connectors were mounted on the back of the instrument case, with the shield wall between input and output pins. This measure was taken to prevent distortion of experimental readings from parasitic coupling and signal noise interference. Ferrite beads were placed on the temperature sensor wires within the instrument case to ensure further protection from common mode noise. Four connectors were employed for testing, all with $50 \Omega$ characteristic impedance: a gold SMA coaxial RF plug, two BNC connectors for current application and voltage sampling, and a 4-pin sensor connector for the temperature signals.

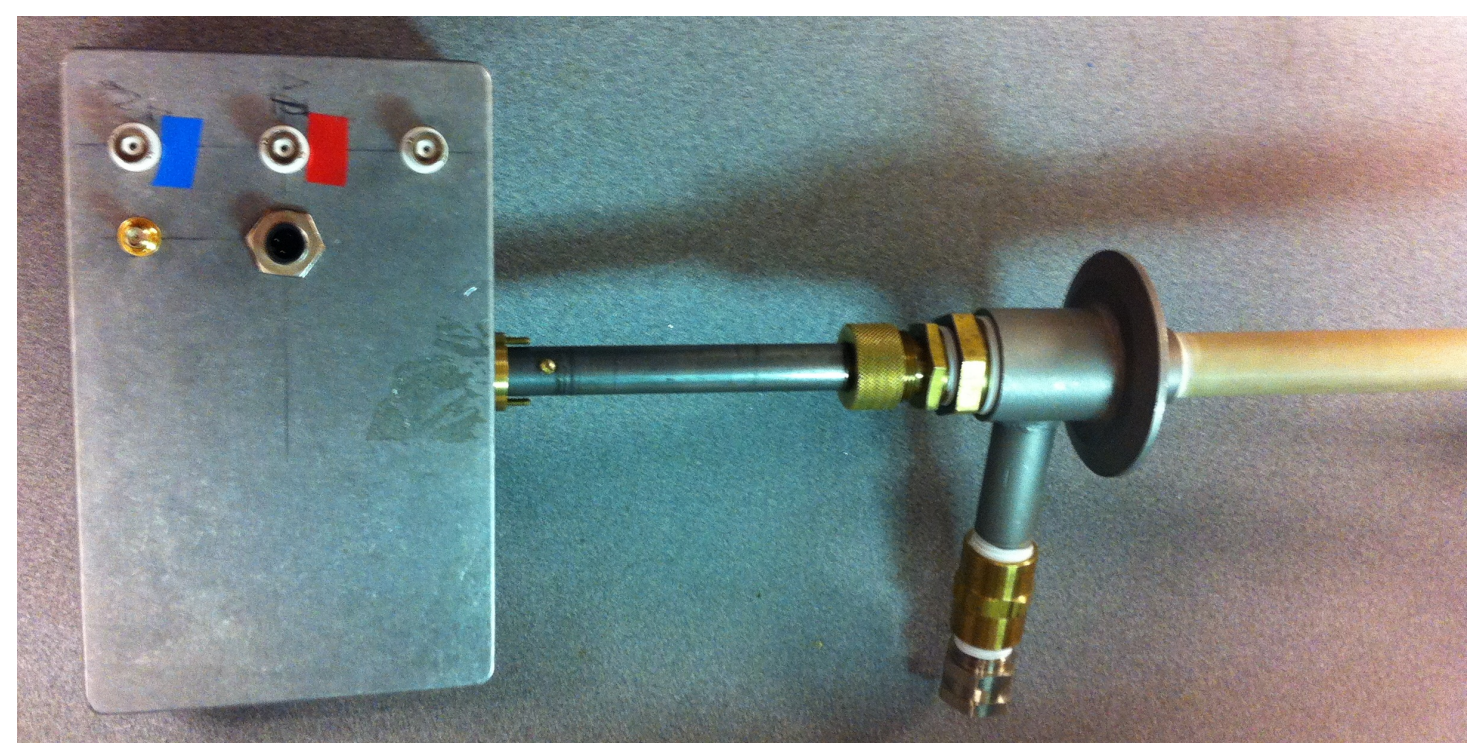

Figure 4.2: Back view of EMI/RFI shield box with connection terminals of the current feed, voltage probe, RF input and temperature sensor shown. 
All wired connections between the junction box and ACC device were soldered at either end and fed through a metal conduit pipe, with an outer fiberglass sheathing tube used as a temperature gradient guard. The fiberglass pipe was fastened to the metal conduit with an adjustable valve, and connected to a dewar cap. The dewar cap was held in position by a clamp fitting, allowing the dipstick to move within the rigid placement of the fiberglass tube at the top of the dewar.

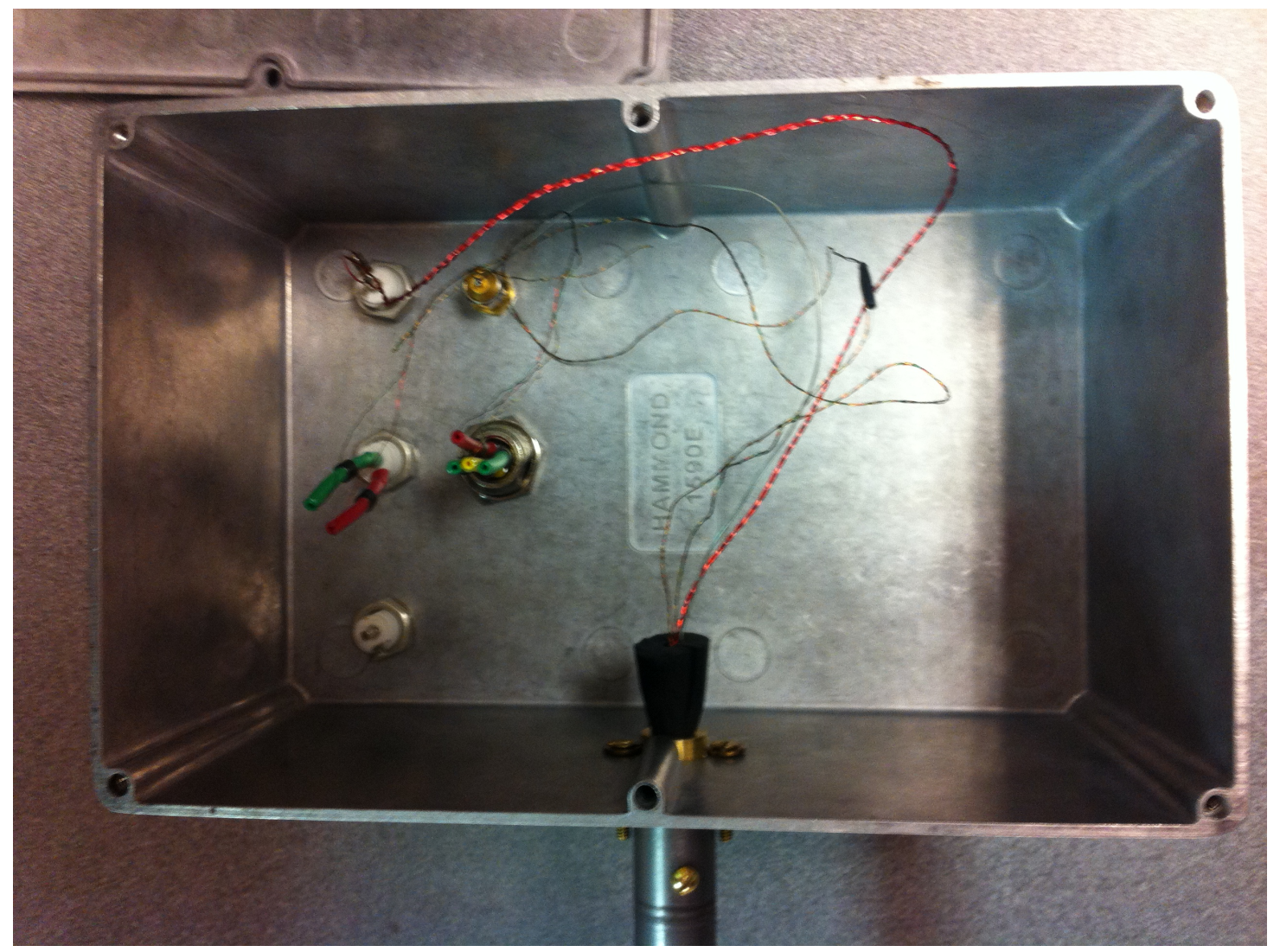

Figure 4.3: Inside view of the EMI/RFI shield box input terminals connected to feed wires; which were led into the metal conduit through a rubber stopper for stability. 
A high frequency Lakeshore cryogenic semi-rigid coaxial cable, with thermal conductivity of $4.5 \mathrm{~W} /(m * K)$ at $4.2 K$, carried RF signal from the input port at the junction box to the inside of the waveguide.[13, 15] The center conductor was 37AWG, silver-plated copperweld steel, and was exposed for a length of $3.14 \mathrm{~mm}$ at the end, acting as a monopole transmit antenna within the waveguide.[13] The transmission length from junction box to waveguide was approximately $1 \mathrm{~m}$, causing estimated insertion losses of $14 \mathrm{~dB}$ at $5 \mathrm{GHz}, 20 \mathrm{~dB}$ at $10 G H z$, and $28.5 d B$ at $20 G H z$.[13] Lakeshore Quad-Twist ${ }^{\mathrm{TM}}$ 32AWG non-ferromagnetic wound 4-wire set of two twisted pairs carried temperature and probed ACC voltage signals, to minimize noise and induced currents along the wires.[12] 22AWG nylon-insulated wound magnet wire with a $3 A$ rating sustained current to the ACC.

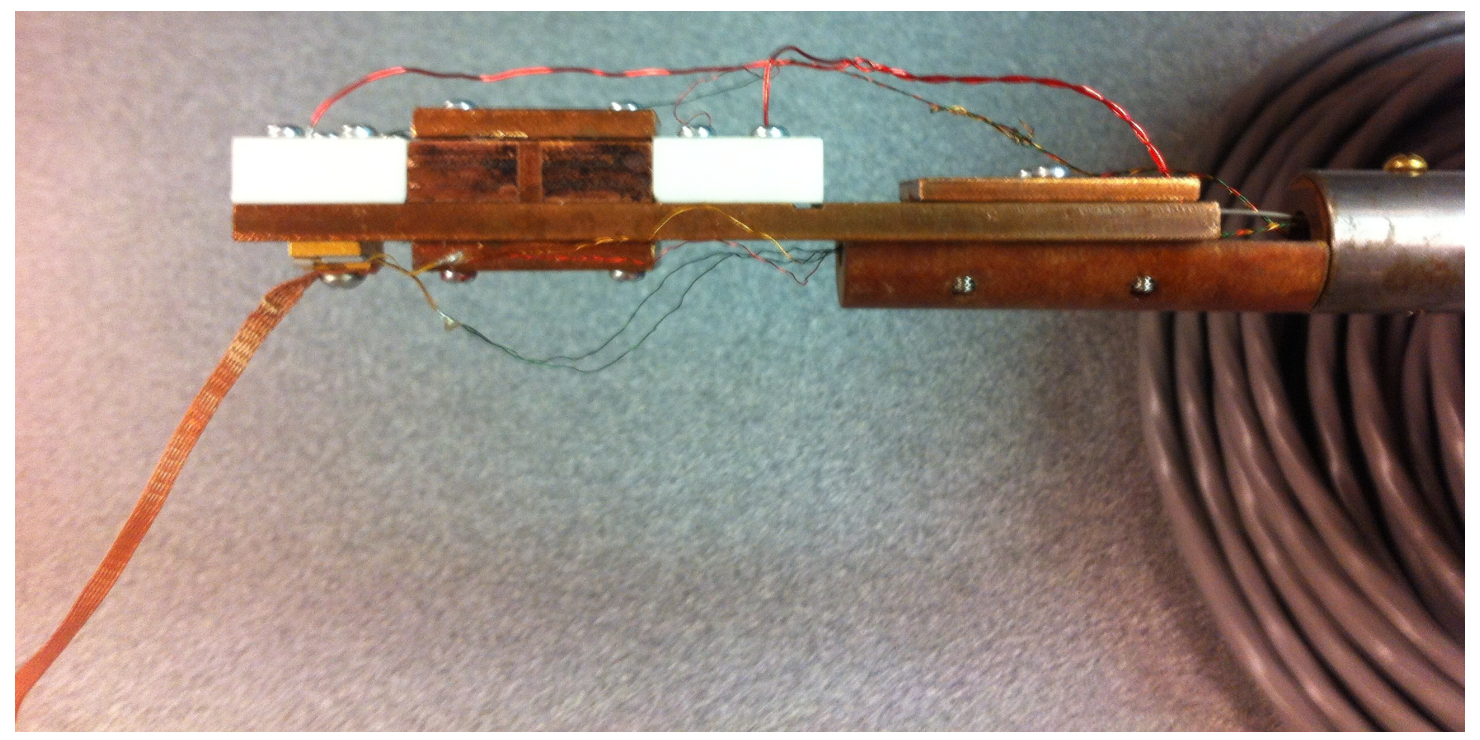

Figure 4.4: Side view of the full assembly, showing four-point ACC probe wires feeding into the Au probes, held in the macor blocks on either side of the waveguide. Below the $\mathrm{Cu}$ holding stage, wires were fed to the DT670A-BO. 
The ACC chip was supported on a copper platform; in the center was machined a $1 \mathrm{~cm}$ diameter hole to facilitate the continuous waveguide, and the top side was machined with $0.520 \mathrm{~mm}$ tall ridges to hold the chip in place. It was connected to the dipstick through a hollow cylindrical insulating phenolic spacer fitting that was held on either side to the dipstick and $\mathrm{Cu}$ platform with stainless steel screws. The phenolic spacer was 0.625 " in diameter and inserted 2" into the bottom of the conduit. It projected a 2" rigid wire guide that was screwed onto the underside of the copper ACC platform. The RF coax was held in place with a machined groove and fastening slab on the top side of the $\mathrm{Cu}$ platform, which led the coax under the ACC chip and into the bottom section of the waveguide (BS1). The bottom waveguide section was capped with a copper block containing a $1 \mathrm{~cm}$ diameter cylindrical cut-out that created $3.89 \mathrm{~mm}$ in total BS1 length when added to the $\mathrm{Cu}$ ACC platform thickness. It was fastened to the copper ACC platform with stainless steel screws. Mounted next to BS1 on the underside of the $\mathrm{Cu}$ platform was a DT670A-BO temperature sensor with an extension of $\mathrm{Cu}$ solder wick braid for thermal stability when the device was held above the He liquid level. The temperature sensor magnet wires were led through the rigid wire support feed of the phenolic spacer on the underside of the $\mathrm{Cu}$ platform.

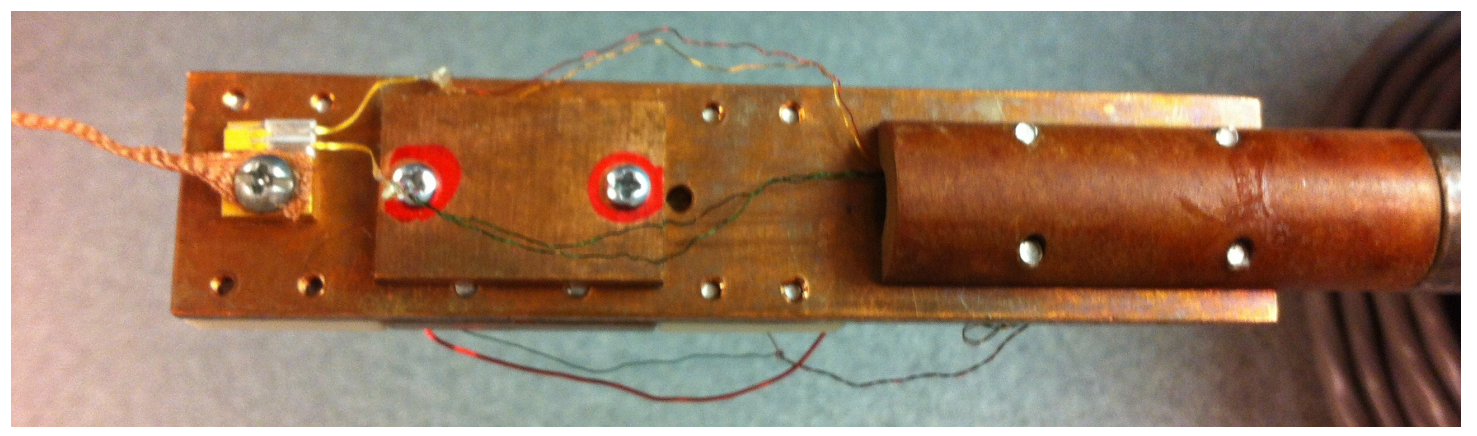

Figure 4.5: Back view of the ACC assembly, showing BS1 and the DT670A-BO. 


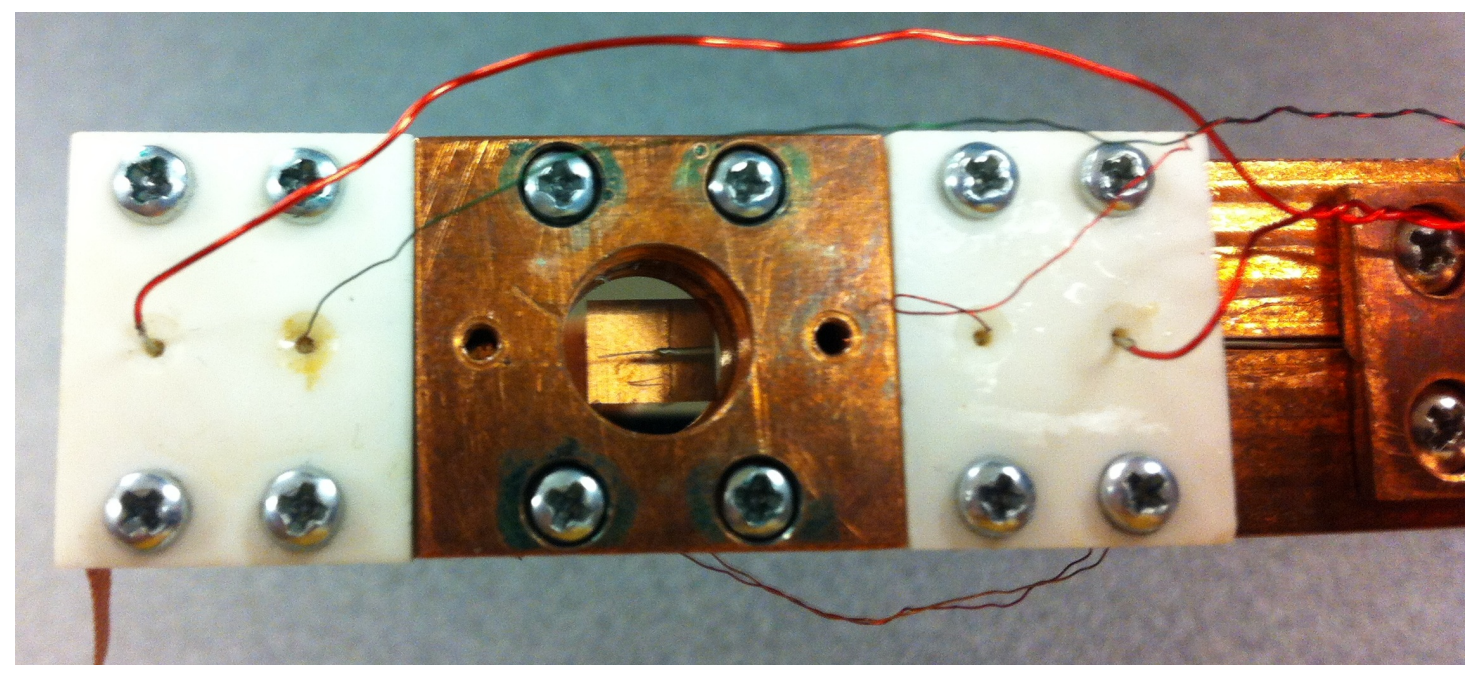

Figure 4.6: Top view of the ACC assembly, shown with the top cap on BS2 removed in order to reveal the inner waveguide.

Four point probe magnet wires were led through the phenolic spacer to Au-plated probes, which were mounted within macor blocks. The macor blocks were screwed into the top side of the $\mathrm{Cu}$ platform, and positioned above the Au pads on the ACC chip. Between the macor blocks was a $6.36 \mathrm{~mm}$ thick $\mathrm{Cu}$ block with a $1 \mathrm{~cm}$ diameter cylindrical hole, positioned above the modified slot antenna on the ACC chip. This was capped by a $\mathrm{Cu}$ slab and served as the top portion of the waveguide (BS2). Preliminary machining drawings are shown in Figure 4.7 and assembly drawings in Figure 4.8, which were drawn by Aaron Datesman and machined by Aric Datesman.[15] 

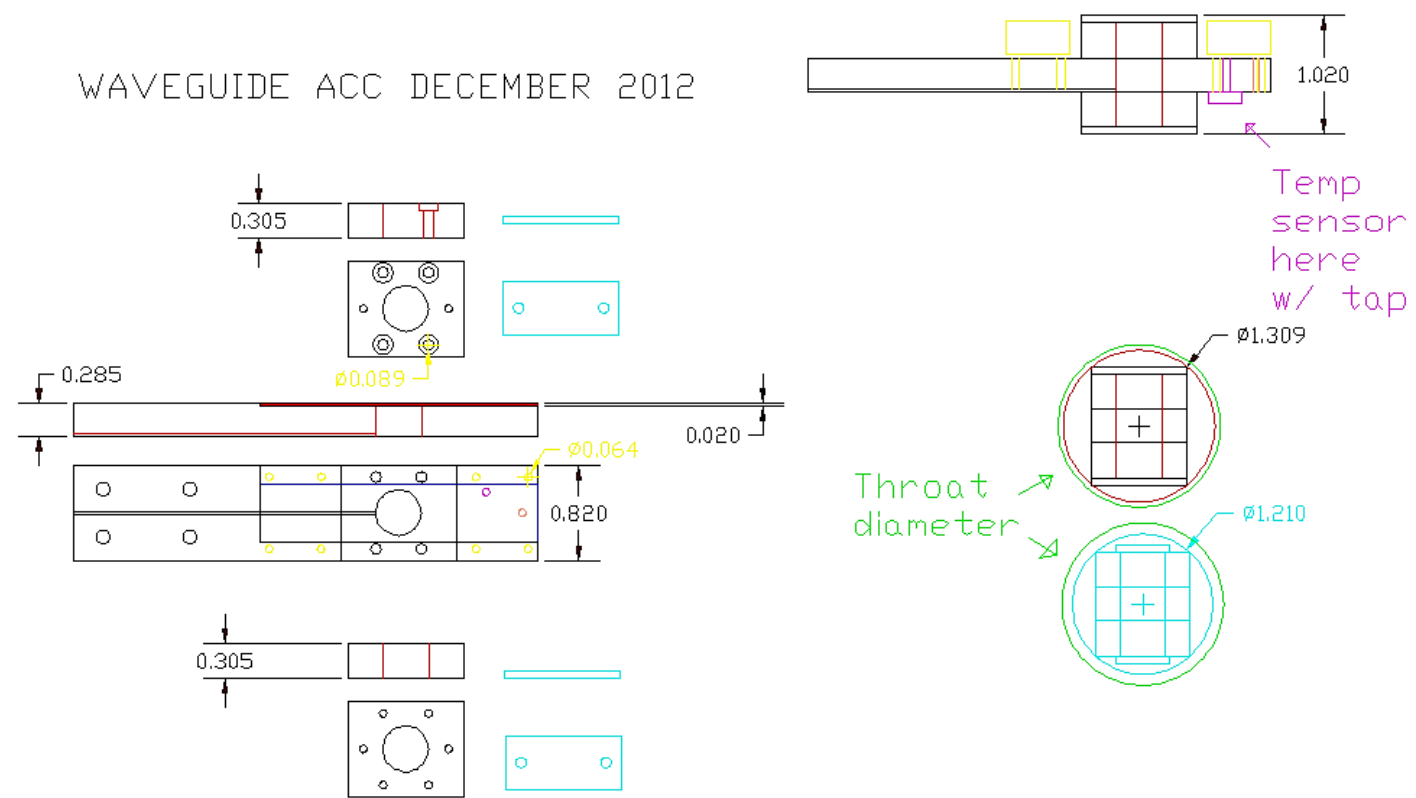

Figure 4.7: Machining CAD schematics for the ACC. Drawn by Aaron Datesman and machined by Aric Datesman
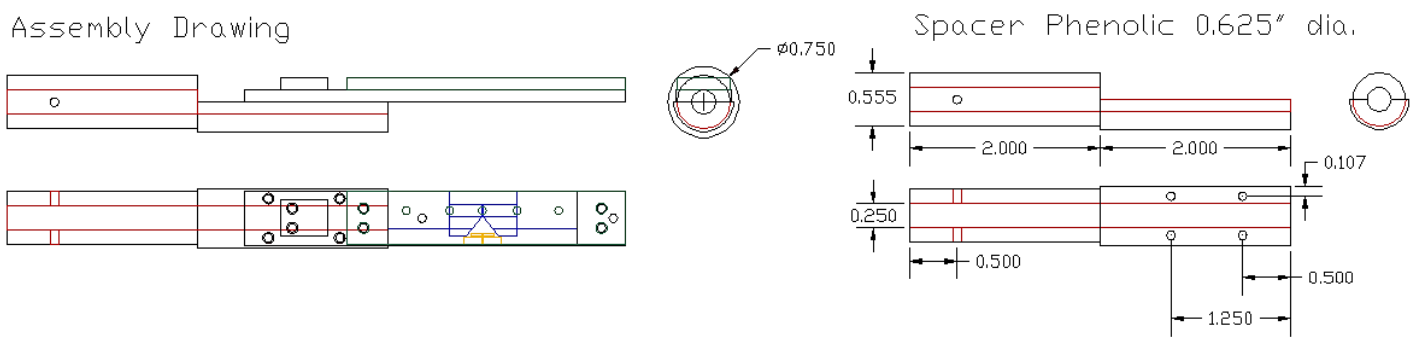

Figure 4.8: Assembly CAD schematics for the ACC. Drawn by Aaron Datesman.

\subsubsection{Auxiliary Elements for Process Control}

Testing preparation required extra steps to ensure control over thermal and electrical processes within the system. This included rejection of atmospheric contents within the LHe dewar; as well as establishment of thermal paths and electrical boundaries. 


\subsubsection{Temperature Cycling}

The dipstick and ACC block were treated with pre- and post- drying cycles for each LHe submersion test, using an industrial blow dryer. This prevented atmospheric condensates from entering the device and LHe dewar. Water inside the dewar and device must be minimized, in order to avoid: formation of ice balls on the dewar reservoir entrance, which can damage the dipstick and create undesirable temperature gradients; ice on ACC probes, which can distort meter readings; ice within the waveguide, which creates an altered RF propagation profile; and rust on metal feed conduit under the fiberglass guard.

Submersion into the LHe was done in monitored increments of time and temperature fluctuation. This ensured that levels were not changing at an excessively rapid pace. Probes can freeze in place and give faulty readings; and the device can crack from lattice deformations if thermal changes exceed the rate of contraction between weak elemental bonds. A basic indicator of overly rapid cooling was faint cracking noises that were emitted as connection points, usually between dissimilar materials, on the device shifted at different rates. The probe was lifted approximately one inch and allowed to settle for 30 seconds when these sounds occurred. The empirical cooling rate limit, garnered from multiple submersion tests, was $\sim 27 K$ per minute. 


\subsubsection{Thermal Conductivity}

Apiezon ${ }^{\mathrm{TM}}$ type $\mathrm{N}$ cryogenic grease coatings were placed between the quartz surface of the ACC chip and the Cu platform; as well as between the temperature sensor, thermal regulating $\mathrm{Cu}$ extension braid and $\mathrm{Cu}$ platform. This formed a thermal conductivity path between components at cryogenic temperatures. A thermal path to the $\mathrm{Cu}$ platform was important in forming a central temperature anchor. In effect the $\mathrm{Cu}$ platform could regulate temperatures in the ACC chip, source heat to the $\mathrm{Cu}$ braid, and also be read accurately by the temperature sensor through sound thermal connections.

\subsubsection{Electrical Isolation}

Clear top-coat nail polish ${ }^{18}$ was employed as an electrical insulator and glue because the acrylates copolymer contents are empirically known to retain material integrity under cryogenic conditions. [4, 15, 30] Nail polish was applied over the soldered connection points between the temperature sensor leads and magnet wire to ensure electrical insulation from the $\mathrm{Cu}$ platform. The bottom of the $\mathrm{Cu}$ block used as BS2 was also covered in a nail polish coating to electrically isolate the conducting thin film from the waveguide. It is important to note that thermal isolation was not guaranteed by the nail polish coating ${ }^{19}$

\footnotetext{
${ }^{18}$ Revlon 950 Extra Life ${ }^{\mathrm{TM}}$

${ }^{19}$ Results of tests with BS2 removed indicated that the thin film may have sourced thermal energy into BS2 when making contact through the nail polish layer.
} 


\subsection{Calibration and Diagnostics}

In addition to controlling thermal and electrical processes, special care was afforded to assure accuracy in sensor readings. Maintenance of the LHe source included grounding the metallic dewar and monitoring pressure release valves to keep the LHe under approximately 1 atmosphere of pressure. The temperature of LHe varies with atmospheric pressure, which became critically important during calibration of the temperature sensor.[29, 30]

\subsubsection{Temperature Sensor}

Temperature recording was based on a sensor diode bias of $10.5 \mu A$. This was prescribed by immersing the Lakeshore DT670A-BO sensor in LHe and adjusting the current supply until a voltage reading $(1.577 \mathrm{~V})$ consistent with a temperature of $4.2 \mathrm{~K}$ was attained.[14] However, the temperature sensor was calibrated for an applied current of $10 \mu A$, meaning that the manufacturer-supplied voltage-temperature characteristics of the device were no longer accurate.[14] Furthermore, the pressure level within the dewar experienced a slight increase when the ACC was active, creating a temperature rise. [29, 30] These conditions indicated that the original heightened temperature readings with a $10 \mu \mathrm{A}$ bias were accurate. This was later confirmed further by the tight accuracy margins of Keithley source/meter devices which minimized the probability of equipment error.[26]

Values of the temperature readings were adjusted through assuming that the crystal lattice of the sensing diode retained a characteristic resistance per temperature level. These characteristic resistances were calculated from original voltage-temperature tables at a bias 
of $10 \mu \mathrm{A}$, and used to inform new expected voltage-temperature values at a bias of $10.5 \mu \mathrm{A}$. Interpolation between listed data and actual readings was initially done through assigning polynomial trend lines to separate temperature regions; and then altered to a cubic spline fit in final temperature calculations for reduced error margins. Temperature calibration is detailed in Appendix E.

\subsubsection{Sources and Meters}

The temperature sensor was biased and metered through a Keithley 2400 SourceMeter ${ }^{\circledR}$ with $0.033 \%+2 n A$ source accuracy and $0.027 \%+700 p A$ measurement accuracy in the $10 \mu A$ range.[26] A Keithley 2420 SourceMeter ${ }^{\circledR}$ performed the four-point-probe operation on the ACC chip. It carried $0.066 \%+20 \mu A$ source accuracy and $0.055 \%+6 \mu A$ measurement accuracy in the $100 \mathrm{~m} A$ range, $0.067 \%+900 \mu A$ source accuracy and $0.066 \%+570 \mu A$ measurement accuracy in the $1 A$ range.[26]

Data recordings were automated with Keithley Instruments LabTracer software, which is programmed to run four intervals of 11 samples with variable user-specified sampling rates. The most rapid sampling rate available was approximately one sample per $10 \mathrm{~ms}$. The meters were coordinated with Labtracer HMI through a NI GPIB-USB-HS adapter and two daisy-chained GPIB cables. Each device carried a unique GPIB address, which was identified and programmed into the software interface for device recognition.[27]

RF radiation was supplied through an Agilent E8241A signal generator with $\pm 0.8 \mathrm{dBm}$ accuracy in the $2-20 \mathrm{GHz}$ range, $0.01 \mathrm{~dB} /{ }^{\circ} \mathrm{C}$ typical temperature stability, and operating temperature of $25^{\circ} \mathrm{C}$.[48] It was pre-calibrated in accordance with age, operating temperature 
and line voltage effects. [40, 48, 55] Signal was carried to the junction box through an RF cable rated up to $24 G H z$, fastened at each terminal with a specified torque wrench to ensure proper connection. [10, 40, 55]

\subsubsection{LHe Level Evaluation}

Acoustic resonance LHe level assessment was performed weekly, in order to account remaining stock and understand the rate of helium evaporation. The science of this method rests on the physical characteristic of sound as a measure of pressure; which can become resonant in an open tube with separate air flow regimes at each end, or effective boundaries to contain standing waves. [6, 51] When one end of a hollow rigid tube is placed at the LHe level, it causes the He to boil by introducing a temperature differential. This boiling creates airflow at the bottom of the cylinder different from that at the top. It also mimics an end boundary at the liquid surface. When the top of the tube is left open, a phase-shifted resonance is established, which materializes a faint sound.[6] When the top of the tube is capped by a finger, end boundaries are established and the tube supports an acoustic resonance. The physical result is a pulsed pressure at the top of the hollow tube which is sensed by the tip of a finger. Noting the point when the pulsed pressure at the top of the tube begins allows inference of the liquid helium level within the dewar.[4] 
Table 4.1: Physical Properties of Tested Samples. Common to all samples are metal deposition thicknesses of $30 \mathrm{~nm} \mathrm{Nb}$ and $50 \mathrm{~nm} \mathrm{Au}$; as well as area dimensions of $2.2 " x 0.47$ ".

\begin{tabular}{ccccc}
\hline \hline $\begin{array}{c}\text { Sample } \\
\text { Identification }\end{array}$ & Qtz Orientation & Qtz Thickness & $\begin{array}{c}\text { Minimum } \\
\text { Transmission Width }\end{array}$ & $\mathrm{I}_{\mathrm{C}}$ \\
\hline A-MS-FT & Amorphous & $0.515 \mathrm{~mm}$ & $2.96 \mathrm{~mm}(2)$ & $1.7 \mathrm{~A}(6.40 \mathrm{~K})$ \\
A-MS-TT & Amorphous & $0.515 \mathrm{~mm}$ & $0.852 \mathrm{~mm}(2)$ & $0.73 \mathrm{~A}(6.40 \mathrm{~K})$ \\
B-MS-FT & X\&Y\&Z & $0.350 \mathrm{~mm}$ & $2.96 \mathrm{~mm}(2)$ & $0.39 \mathrm{~A}(7.08 \mathrm{~K})$ \\
B-TL-0 & X\&Y\&Z & $0.350 \mathrm{~mm}$ & $1.15 \mathrm{~mm}(1)$ & $0.44 \mathrm{~A}(6.98 \mathrm{~K})$ \\
\hline
\end{tabular}

\subsection{Testing Processes}

The following sections detail testing scenarios to assess several configurations of the physical ACC device in varied applied field conditions. General analysis techniques are discussed separately, followed by a cumulative review of conditions for individual tests in tabular form.

\subsubsection{ACC Chip Variation}

Four separate chip samples were tested, as shown in Table 4.1. The $I_{C}$ values could not be taken at the original minimum allowable LHe temperature for two of the samples because of low helium levels within the dewar; their sampling temperatures are noted in parenthesis. Minimum transmission width has a number next to it indicating the multiple for the full width dimension; the modified slot multiple is (2) and the single thin transmission strip-line is (1). This distinction is important because the full transmission width correlates with the maximum possible current density in the device.

ACC chips were varied in an effort to test functional effects of the quartz substrate, receive antenna dimensions, and maximum transmission width of the thin film $\mathrm{Nb}$. It was 
postulated that quartz substrate lattice orientation and thickness would create deviations in signal reflection characteristics and thermal stability. Receive antenna dimensions were varied from the original modified slot antenna to a simple $1.15 \mathrm{~mm}$ wide transmission stripline, in order to assess comparative coupling influences. Maximum thin film transmission width was trimmed in order to establish a lower critical current density, which was expected to promote fully-normal state actuation. The chip identified as A-MS-FT in Table 4.1 was used as a control sample, to which all tests were applied ${ }^{20}$ Identities of the samples under test are noted for each of the following testing method descriptions.

\subsubsection{Base Critical Current - Full Submersion}

All samples were tested to establish their base critical current. This was done at full submersion of the device into the LHe dewar, thereby holding it at the coldest possible temperature level. As noted in Section 4.3.1 and Table 4.1, the base temperature varied between the A and B chips. Two operative methods were used to assess the critical current, both done in the absence of RF radiation: 1) gradual, manually adjusted current increases and 2) automated fast current sweeps, noting the normal transition point. The manual adjustments, if made too slowly, gave lower $I_{C}$ values than the automated sweeps. This was due to time-coupled thermal penetration at higher current levels. In the absence of excess thermal penetration, results from the two methods showed good agreement in $I_{C}$ values. The automated sweep sampling rate was approximately $10 \mathrm{~mA} / 10 \mathrm{~ms}$ and the manual threshold rate for comparative equivalency was approximately $10 \mathrm{~mA} / 0.5 \mathrm{~s}$.

\footnotetext{
${ }^{20}$ This carries an exception with room temperature S11 analysis because the chip shattered upon removal from the LHe dewar after the final submersion test was completed, and before S11 data was taken.
} 


\subsubsection{Current Sweep Profile}

Swept currents between $0 A$ and $\sim 1.25 I_{C}$, at a rate of 1 sample per $10 \mathrm{~ms}$, were applied at full submersion to samples: A-MS-FT, B-MS-FT and B-TL-0. This was done with and without applied RF radiation, in order to assess comparative normal transition developments. Critical transition current and temperature will deviate from a base RF-absent transition in a particular manner, depending on the specific RF effects. These comparative sweeps allowed inspection through the transition current level, as well as the temperature of transition, into the type of quasi-particle and super-current manipulation occurring. Notable in these tests were points of transition suppression with applied RF, which will be discussed in subsequent chapters 21

\subsubsection{Current Level Steps}

Early tests on the control chip, A-MS-FT, were repeated at stepped current levels ranging from $<<I_{C}$ to $\sim 0.9 I_{C}$. This illustrated differences in super-current dynamics and transition properties within close range of $I_{C}$, as opposed to a low current control test. These separate current level distinctions carry different active quasi-particle conditions, which could then be studied in the presence of a waveguide RF field application. [49]

\subsubsection{RF Switching Step Intervals}

Stepped RF switching intervals were recorded after establishment of the frequency level corresponding with the highest voltage rise across the ACC. Frequency was optimized

\footnotetext{
${ }^{21}$ Appearance of the phenomenon will be shown in Chapter 5, with accounted parameters on comparative plots. Refer to Chapter 6 for corollaries of normal transition suppression; as well as altered transition behavior.
} 
through a preliminary manual $1 G H z-20 G H z$ sweep with $100 M H z$ resolution. Each test had 4 intervals consisting of 11 samples. Odd-numbered sampling intervals were absent of RF and even-numbered intervals were subjected to RF radiation. Sequential interval RF identification was then, off-on-off-on. These switching intervals were applied to display the highest induced voltage levels from which the ACC could recover back into the superconducting state when RF was removed.

\subsubsection{Transmit Antenna Placement}

Three extension lengths of the RF transmitting coaxial cable within the waveguide were observed. The cable was trimmed to expose $3.14 \mathrm{~mm}$ of the inner conductor, which was held constant through all tests. Originally, the end of the outer coax sheathing was placed flush with the wall of the waveguide, and only the exposed $3.14 \mathrm{~mm}$ of inner conductor extended into the waveguide. Subsequent tests incorporated insertion lengths with outer coax sheathing also extended into the waveguide. Two altered coaxial penetrations into the waveguide were tested, with total insertion lengths of $5.15 \mathrm{~mm}$ and $7.28 \mathrm{~mm}$. This revealed functional effects from varied placement of the exposed $3.14 \mathrm{~mm}$ transmit section in relation to the receive slot; and also of a length of grounded metallic stub within the waveguide. All samples were tested with a $5.15 \mathrm{~mm}$ extension length, and only A-MS-FT was tested at the other two lengths. 


\subsubsection{Temporal Trends Near $I_{C}$}

Sample A-MS-FT was fully submerged in the LHe dewar for a minimum temperature bias, with a $3.14 m m$ total coaxial extension into the waveguide. Tests took 44 samples with RF on and then 44 samples with RF off, holding all other controlled variables constant. The RF was set to $18.86 \mathrm{GHz}$. This served a comparison between time-dependent effects of RF radiation and $\mathrm{RF}$ absence.

Progressive data sets were taken at different applied constant current levels, each subsequent test approaching closer to $I_{C}$. The zero time point was set by the timestamp of the first reading for each test run. All tests were identically configured, and differences in time were caused by small clock fluctuations in sampling. Datapoints were plotted against their own individual timestamps, to account the actual time passed with any sampling clock fluctuations, rather than the sequential sample number.

At the time instant of $0^{-}$all components were off. At $0^{+}$the LabTracer software applied bias current and began sampling at a rate of 1 sample every $\sim 10 \mathrm{~ms}$. RF tests were run with manual actuation at the time when software was engaged, to establish a uniform zero-point for all operations.

\subsubsection{Frequency Effect Sweeps}

All samples were held at a set submersion level, to correspond with temperature range biasing, then exposed to swept RF frequencies with $30 \mathrm{dBm}$ applied power at the junction box. The swept range of frequencies was $1 G H z-20 G H z$ with $100 M H z$ resolution. Values 
were sequentially swept through a manual dial to assess high voltage-inducing frequencies and their respective bandwidths. High induced voltage bandwidths were subject to backward and forward sweeps to account lag momentum effects which artificially expand voltage inducing frequency bandwidths. It is notable that the insertion loss of the RF coax is proportional to applied frequency, meaning that higher frequencies were delivered into the waveguide at lower power levels.

\subsubsection{Frequency Level Steps}

Independent stepped frequency level tests were taken over the $1 G H z-20 G H z$ interval with a $1 G H z$ resolution for all ACC chips. Each frequency level was applied for the course of 11 samples, with isolation between RF application intervals. These applications show the effects caused by individual frequencies, without the threat of momentum lag introduced from sequentially swept samples.

\subsubsection{Temperature Effect Steps}

Frequency applications on all samples were run at steps within a range of bias temperature submersion placements. This showed induced voltage profiles and superfluid dynamics with applied RF far below $T_{C}$, in close range below $T_{C}$, at $T_{C}$ and above $T_{C}$. Interactions between the superfluid and RF field materialized in different ways, depending on the bias temperature level. 


\subsubsection{Temperature Tracking}

Each bias temperature submersion point carried with it a bandwidth of induced temperature variation. Trends within the bias temperature point bandwidth were plotted in parallel with their time-correspondent voltage data to show the interaction between critical conditions for each datum recording. This tracking was essential because RF magnetic field penetration into a thin film superconductor at temperatures in close range of $T_{C}$ has the potential to alter the energy gap and cause thermodynamic deviations, rather than induce voltages. [49]

\subsubsection{Power Level Evaluation}

Carrier power levels of RF signal were swept from $1 \mathrm{dBm}-30 \mathrm{dBm}$ with a $1 \mathrm{dBm}$ resolution for all chips. Sweeps were biased at the optimized voltage-inducing frequency level of a given chip to find the point where power was strong enough to spur a rise in voltage. Also assessed were the voltage trends at increasing power levels.

\subsubsection{Waveguide Alteration}

Most submersion tests were operated with the full waveguide assembled in accordance with device design. However, two tests were run on A-MS-FT with altered waveguide assemblies. These involved the removal of BS1 and BS2 respectively, to investigate the function of waveguide components and which frequencies they supported. 


\subsubsection{Shunt Impedance}

Parallel resistive loads with varied ohmic values at room temperature were connected to input terminals of the testing apparatus using A-MS-FT. This was done in an effort to redirect current at the switching point and avoid thermal runaway, which was causing a latch-to-normal conductivity at sufficiently high current levels. The redirection created a current divider; true redirection would require a flip-flop switching mechanism.

\subsubsection{S11 at Room Temperature}

S11 analysis was undertaken at room temperature on a vector network analyzer (VNA) with maximum power output of $10 \mathrm{dBm}$, using a rated $18 \mathrm{GHz}$ limit cable. Results were questionable because the power level was one-third that used in LHe submersion tests, and the cable was insufficient at the highest frequencies. Also, the dielectrics of substrates within the waveguide carry lower values at room temperature in comparison to cryogenic conditions. This indicates slight alterations in propagation parameters and resonance of the device at room temperature. 
Table 4.2: Conditions and Examination per Test Scenario. Examination techniques are identified with their corresponding subsection heading numbers.

\begin{tabular}{|c|c|c|c|c|c|}
\hline $\begin{array}{l}\text { Testing } \\
\text { Date }\end{array}$ & $\begin{array}{c}\text { Waveguide } \\
\text { Configuration }\end{array}$ & $\begin{array}{l}\text { Total RF Transmit } \\
\text { Coaxial Extension }\end{array}$ & $\begin{array}{c}\text { Sample } \\
\text { Under Test }\end{array}$ & $\begin{array}{c}\text { Examination } \\
\text { Technique }\end{array}$ & $\begin{array}{l}\text { Examination } \\
\text { Value Range }\end{array}$ \\
\hline $10 / 8 / 2013$ & Full & $3.14 \mathrm{~mm}$ & A-MS-FT & \begin{tabular}{|l|}
4.3 .2 \\
4.3 .3 \\
4.3 .10 \\
\end{tabular} & $\begin{array}{c}\text { none } \\
0 \mathrm{~A}-0.25 \mathrm{~A} \\
4.2 \mathrm{~K}-9 \mathrm{~K}\end{array}$ \\
\hline $10 / 9 / 2013$ & Full & $3.14 \mathrm{~mm}$ & A-MS-FT & 4.3 .5 & $\begin{array}{l}15.67 \mathrm{GHz} \\
4.2 \mathrm{k}-9 \mathrm{~K}\end{array}$ \\
\hline $10 / 28 / 2013$ & Full & $3.14 \mathrm{~mm}$ & A-MS-FT & \begin{tabular}{|l|}
4.3 .5 \\
4.3 .7 \\
4.3 .11 \\
\end{tabular} & $\begin{array}{c}18.86 \mathrm{GHz} \\
0 \mathrm{~s}-1.6 \mathrm{~s} \\
4.24 \mathrm{~K}-4.29 \mathrm{~K}\end{array}$ \\
\hline $10 / 29 / 2013$ & Full & $7.28 \mathrm{~mm}$ & A-MS-FT & $\begin{array}{l}4.3 .5 \\
4.3 .4\end{array}$ & $\begin{array}{c}4 \mathrm{GHz} \\
19.1 \mathrm{GHz} \\
0.5 \mathrm{~A}, 0.17 \mathrm{~A}, 0.46 \mathrm{~A} \\
1.33 \mathrm{~A}\end{array}$ \\
\hline $10 / 30 / 2013$ & Full & $7.28 \mathrm{~mm}$ & A-MS-FT & 4.3 .5 & $\begin{array}{l}4 \mathrm{GHz} \\
0.5 \Omega\end{array}$ \\
\hline $11 / 6 / 2013$ & Full & $7.28 \mathrm{~mm}$ & A-MS-FT & \begin{tabular}{|l|}
4.3 .4 \\
4.3 .8 \\
4.3 .9 \\
4.3 .10 \\
4.3 .11 \\
\end{tabular} & $\begin{array}{c}0.05 \mathrm{~A}-1.5 \mathrm{~A} \\
1 \mathrm{GHz}-15 \mathrm{GHz} \\
1 \mathrm{GHz}-20 \mathrm{GHz} \\
5.7 \mathrm{~K}-6.1 \mathrm{~K} \\
5.9 \mathrm{~K}-11 \mathrm{~K}\end{array}$ \\
\hline $11 / 12 / 2013$ & Full & $7.28 \mathrm{~mm}$ & A-MS-FT & \begin{tabular}{|l|}
4.3 .14 \\
4.3 .11 \\
\end{tabular} & $\begin{array}{l}0.64 \Omega-3 \Omega \\
4.2 \mathrm{~K}-5.4 \mathrm{~K}\end{array}$ \\
\hline $11 / 14 / 2013$ & Full & $5.15 \mathrm{~mm}$ & A-MS-FT & \begin{tabular}{|l|}
4.3 .8 \\
4.3 .10 \\
4.3 .11 \\
\end{tabular} & $\begin{array}{c}1 \mathrm{GHz}-20 \mathrm{GHz} \\
5.9 \mathrm{~K}-6.2 \mathrm{~K} \\
6.00 \mathrm{~K}-6.14 \mathrm{~K}\end{array}$ \\
\hline $11 / 18 / 2013$ & Omit BS1 & $5.15 \mathrm{~mm}$ & A-MS-FT & \begin{tabular}{|l|}
4.3 .8 \\
4.3 .10 \\
4.3 .11 \\
\end{tabular} & $\begin{array}{c}1 \mathrm{GHz}-20 \mathrm{GHz} \\
5.9 \mathrm{~K}-6.2 \mathrm{~K} \\
6.00 \mathrm{~K}-6.25 \mathrm{~K}\end{array}$ \\
\hline $11 / 19 / 2013$ & Full & $5.15 \mathrm{~mm}$ & A-MS-TT & \begin{tabular}{|l|}
4.3 .8 \\
4.3 .10 \\
4.3 .11 \\
4.3 .12 \\
\end{tabular} & $\begin{array}{c}1 \mathrm{GHz}-20 \mathrm{GHz} \\
5.9 \mathrm{~K}-6.2 \mathrm{~K} \\
6.00 \mathrm{~K}-6.25 \mathrm{~K} \\
1 \mathrm{dBm}-30 \mathrm{dBm}\end{array}$ \\
\hline $11 / 20 / 2013$ & Full & $5.15 \mathrm{~mm}$ & $\begin{array}{l}\text { B-MS-FT } \\
\text { B-TL-0 }\end{array}$ & \begin{tabular}{|l|}
4.3 .8 \\
4.3 .10 \\
4.3 .11 \\
4.3 .12 \\
\end{tabular} & $\begin{array}{c}1 \mathrm{GHz}-20 \mathrm{GHz} \\
5.9 \mathrm{~K}-6.2 \mathrm{~K} \\
6.00 \mathrm{~K}-6.25 \mathrm{~K} \\
1 \mathrm{dBm}-30 \mathrm{dBm}\end{array}$ \\
\hline $11 / 23 / 2013$ & Omit BS2 & $5.15 \mathrm{~mm}$ & A-MS-FT & \begin{tabular}{|l|}
4.3 .8 \\
4.3 .10 \\
4.3 .11 \\
4.3 .12 \\
\end{tabular} & $\begin{array}{c}1 \mathrm{GHz}-20 \mathrm{GHz} \\
5.9 \mathrm{~K}-6.2 \mathrm{~K} \\
6.00 \mathrm{~K}-6.25 \mathrm{~K} \\
1 \mathrm{dBm}-30 \mathrm{dBm}\end{array}$ \\
\hline $11 / 27 / 2013$ & $\begin{array}{c}\text { Full } \\
\text { Omit BS1 } \\
\text { Omit BS2 } \\
\text { Removed }\end{array}$ & $5.15 \mathrm{~mm}$ & $\begin{array}{l}\text { A-MS-FT } \\
\text { A-MS-TT } \\
\text { B-MS-FT } \\
\text { B-TL-0 }\end{array}$ & \begin{tabular}{|l|}
4.3 .13 \\
4.3 .15 \\
\end{tabular} & $\begin{array}{c}\text { none } \\
1 \mathrm{GHz}-20 \mathrm{GHz}\end{array}$ \\
\hline
\end{tabular}




\section{Chapter 5: Results}

Compelling outcomes from the experiments outlined in Table 4.2 are reported in this chapter. Graphical representations of experimental results take some liberties in the terms of evaluation. Voltage arose from $\mathrm{RF}$ effects within the $\mathrm{Nb}$ strip. This was a result of electrochemical potential deviations caused by the RF bombardment.[15, 49, 53] When the device latched and reached voltages on the level of $\sim 10 \mathrm{~V}$, an electrical resistance was formed. However, in the cases of very small voltage fluctuations, electrical resistance was not the physical basis of the phenomenon since the device was not in a normal conducting state. A subset of comparative plots between varied device configurations were posed in terms of resistance in order to normalize energy contributions from disparate current applications. It should be noted that a resistance model is only illustrative and not asserted as an accurate portrayal of the quantum processes within the $\mathrm{Nb}$.

\subsection{Quasi-particle Redistribution}

Results involving quasi-particle disequilibrium were noticeable in the temporal tests. A small change in the timing of deviations between the chemical potential on the even- and odd- mode branches was noticed in these tests. 


\subsubsection{Temporal Trends Near $I_{C}$}

The graphs showing a comparative earlier voltage rise with applied RF, exemplified in Figure 5.1. all displayed the same temperature trends: initial comparative temperature suppression with RF on, followed by a crossing of the off/on temperature levels, then a comparatively higher temperature with RF on at the point of voltage rise. This trend is shown in Figure 5.2 . In the plots lacking this temperature profile, as seen in Figure 5.4, voltage rise with RF on is not enhanced. Particularly in the test run at $1.445 \mathrm{~A}$, the voltage rise with RF on is actually suppressed, shown in Figure 5.3

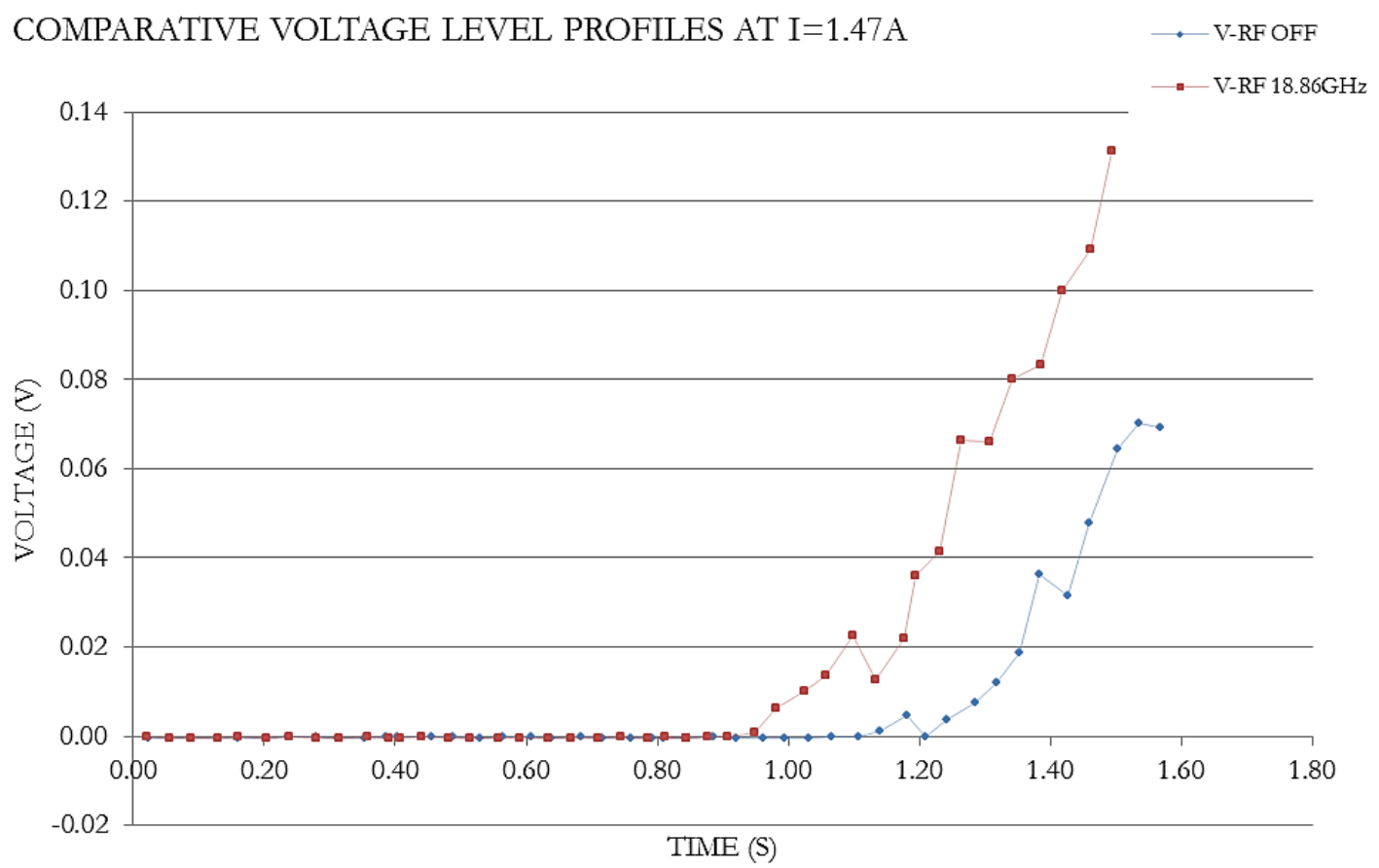

Figure 5.1: Induced voltage comparison at the design frequency in close range of $I_{C}$ indicated signs of energy gap depression, which is a function of even-mode energy perturbation in quasi-particles. 


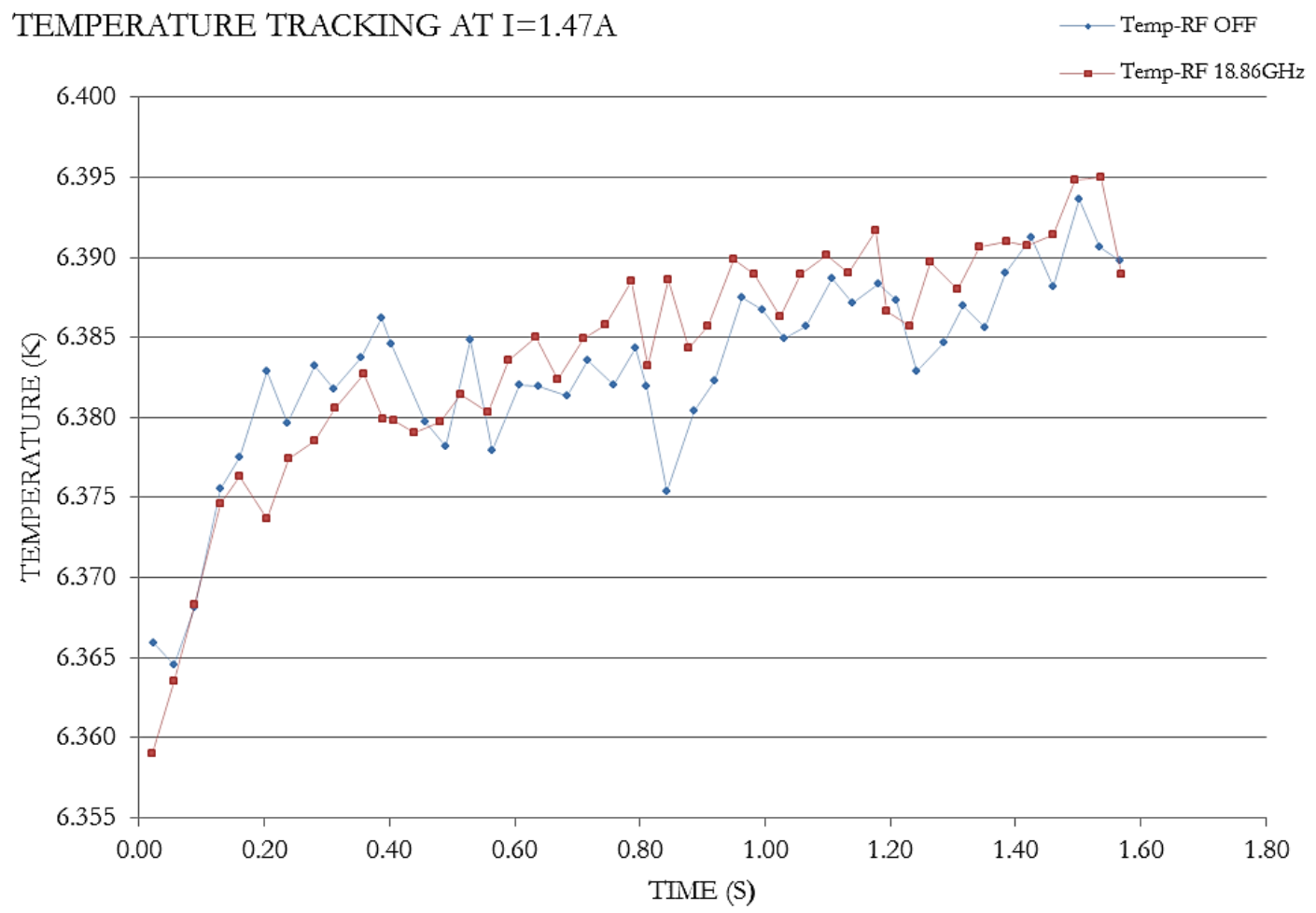

Figure 5.2: Temperature profile for the $1.47 \mathrm{~A}$ application evidencing energy gap depression.

Results shown in Figures 5.1 through 5.4 are very noisy, and subject to significant error margins. The data require further inquiry in terms of accuracy, and also do not show trends of any significant usefulness for switching applications in the present form. 


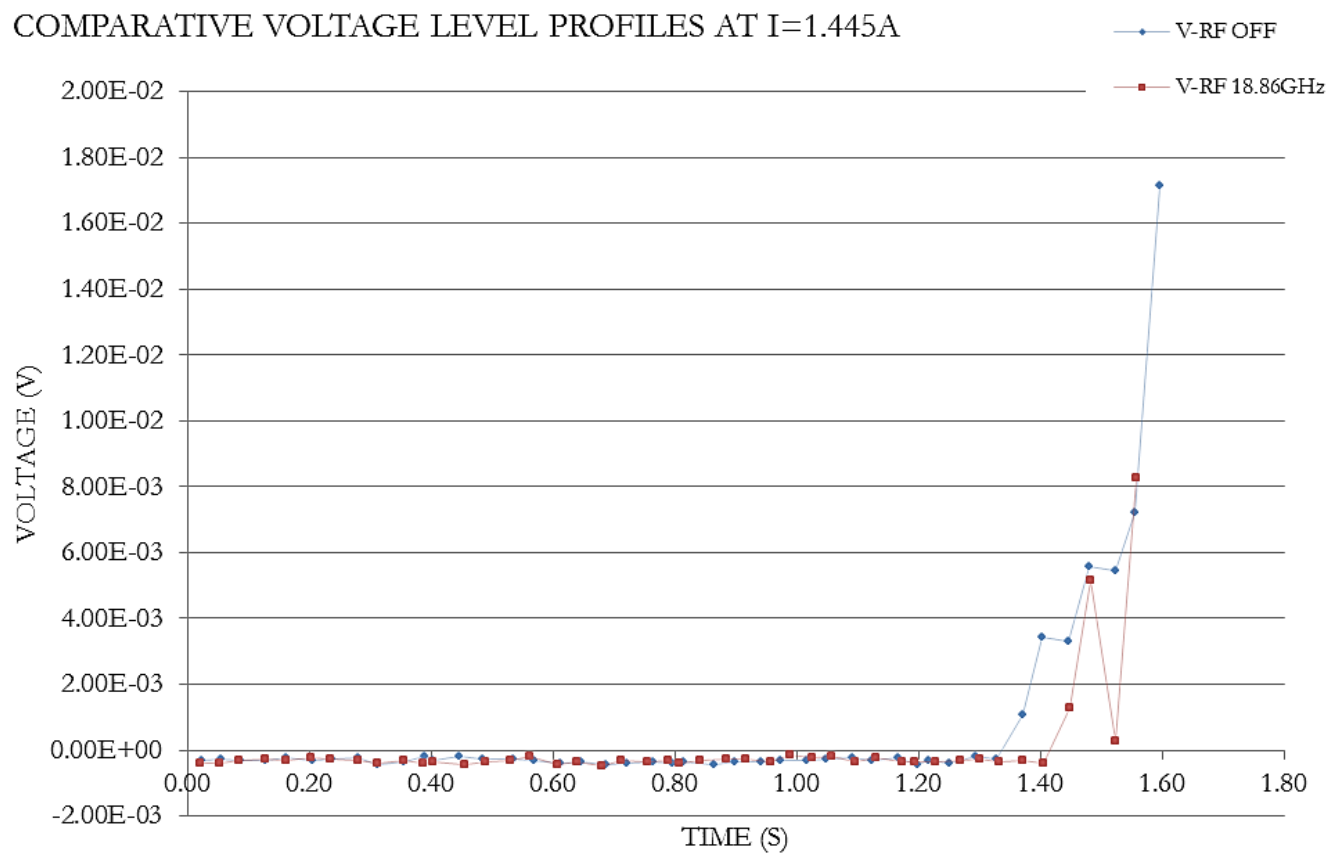

Figure 5.3: Induced voltage comparison at the design frequency sufficiently far below $I_{C}$ indicated signs of energy gap enhancement, a function of even-mode energy perturbation in quasi-particles.

TEMPERATURE TRACKING AT I=1.445A $\rightarrow$ Temp-RF OFF

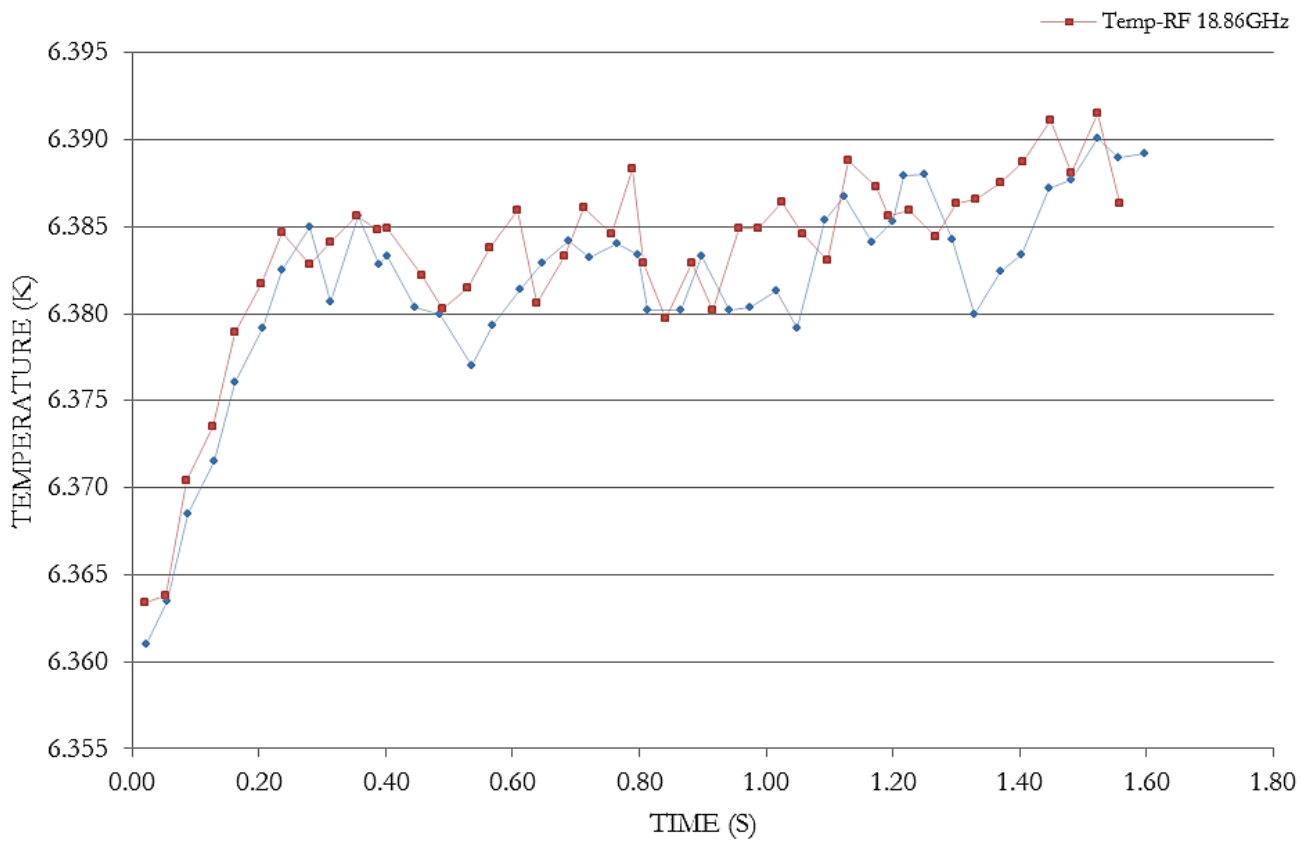

Figure 5.4: Temperature profile for the $1.445 \mathrm{~A}$ application evidencing energy gap enhancement. 


\subsection{Flux Lattice Effects}

Most significant of the experimental observations were voltages on the order of $m V$, arising at frequencies below the waveguide cutoff. Two major voltage peaks, at $4 G H z$ and $9 G H z$, were repeated through several testing configurations.

\subsubsection{Establishment of Switching Condition}

Initial observations of the evanescent waveguide operation phenomenon were made with the monopole transmit antenna extension into the waveguide set at $7.28 \mathrm{~mm}$, maintaining the original $3.14 \mathrm{~mm}$ exposed inner conductor. This extension was made in an effort to align the send and receive ports within closer proximity. ${ }^{22}$ Initially, frequency sweeps were applied at $15 G H z-20 G H z$, and the first indication of induced voltage was seen at $15 G H z$, with the elongated monopole placement.

Figures 5.5 and 5.6 illustrate initial observations at $15 \mathrm{GHz}$ in terms of normalized resistance during RF switching events at multiple applied current levels. A clear transition mechanism emerged from these results; with standard RF induced resistance in the range of $\sim 5-10 m \Omega$, for controlled conditions in the absence of thermal runaway. The large resistance deviations seen in the outlier data show a slight excess of absorbed energy, which was spurring the early stages of thermal runaway.

\footnotetext{
${ }^{22} \mathrm{~A}$ secondary effect of this positioning was excitation of a slot-line resonance, discussed in chapter 6.
} 


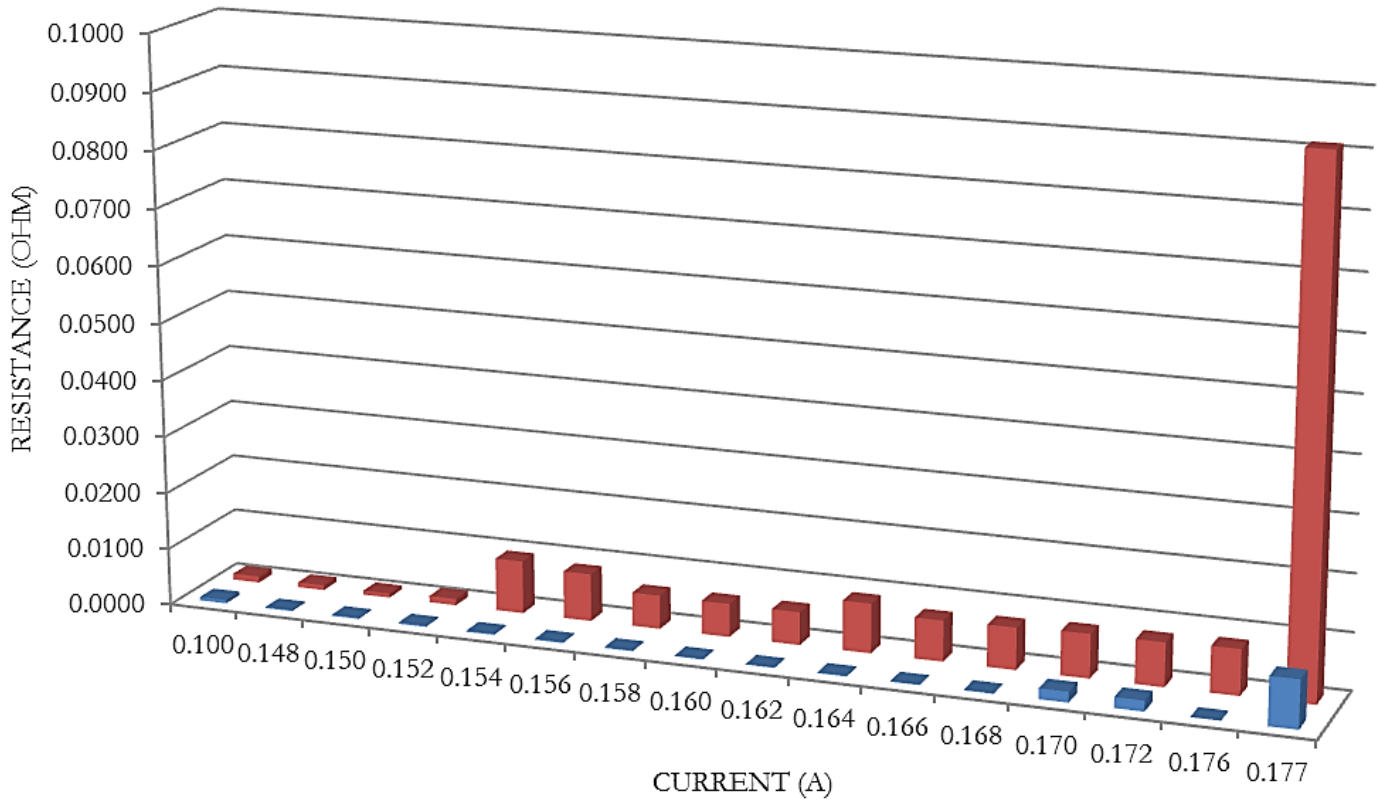

Figure 5.5: Bar graph comparison of normalized resistance values at $15 G H z$ with applied- and absent- RF radiation. The total transmit monopole antenna extension was $7.28 \mathrm{~mm}$. 


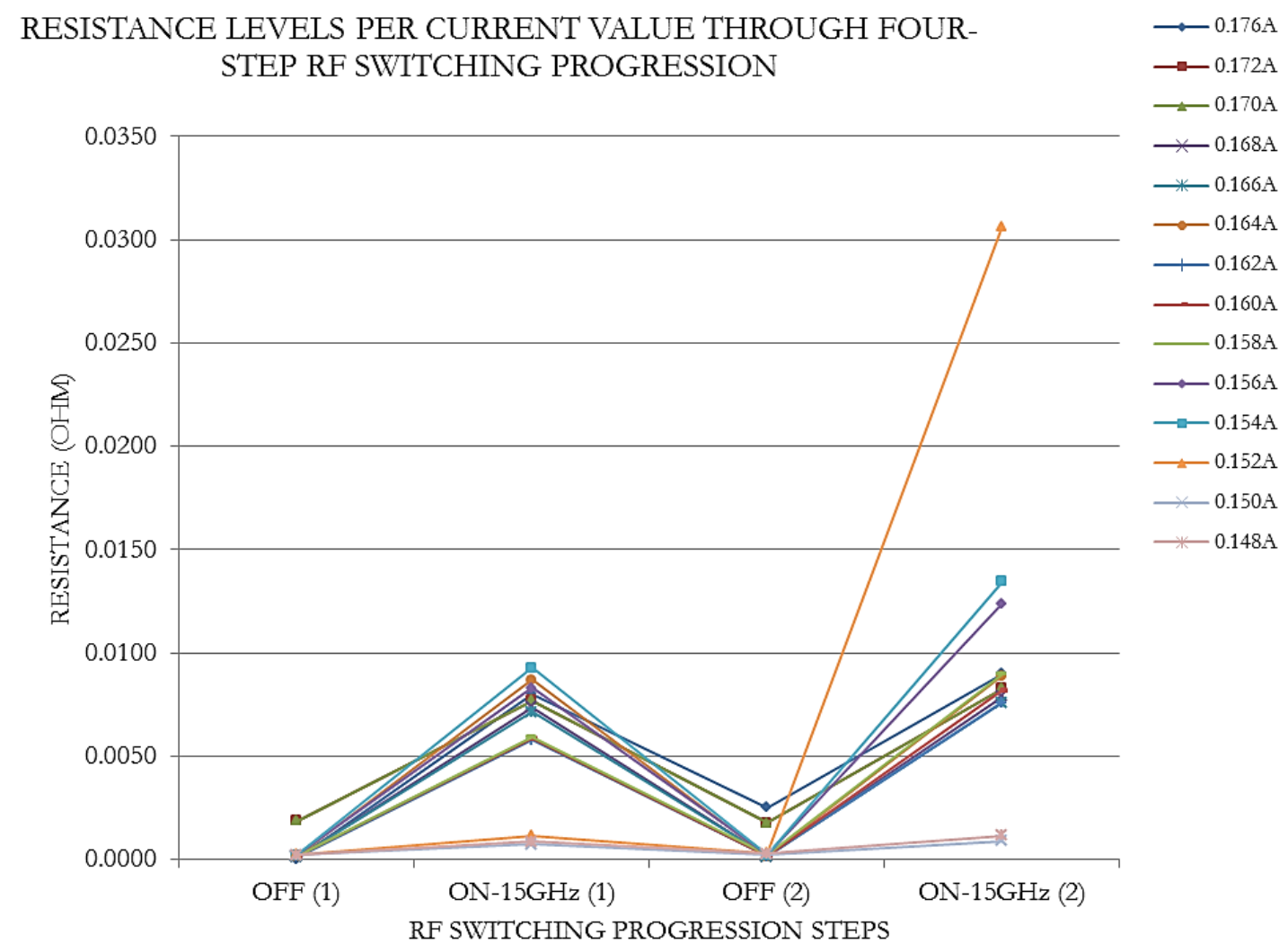

Figure 5.6: OFF-ON-OFF-ON RF switching progression comparison of normalized resistance values at $15 \mathrm{GHz}$ established that the voltage development was reversible. The total transmit monopole antenna extension was $7.28 \mathrm{~mm}$. 
After discovering induced voltages at $15 \mathrm{GHz}$, below the waveguide cutoff, an inquiry of evanescent mode resonance emerged. In addressing this, frequencies from $1 G H z-20 G H z$ were swept through the device with a resolution of $100 \mathrm{M} \mathrm{Hz}$. The highest induced voltages in these sweeps occurred at $4 G H z$, with controlled normalized resistance in the range of $50-60 m \Omega$, shown in Figure 5.7

\section{RESISTANCE LEVELS AT EIGENMODE RESONANCE 4GHZ THROUGH FOUR-STEP RF SWITCHING PROGRESSION}

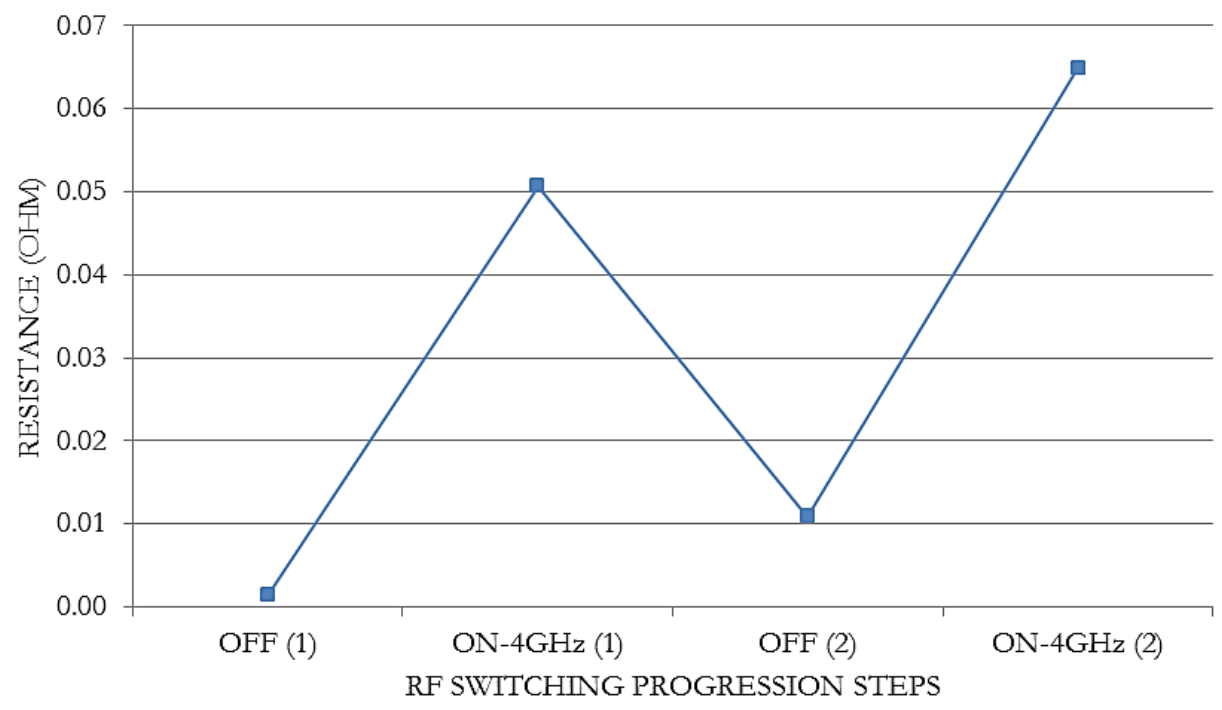

Figure 5.7: Establishment of the dominant voltage developing frequency at $4 \mathrm{GHz}$ with a $7.28 \mathrm{~mm}$ total transmit monopole antenna extension. 

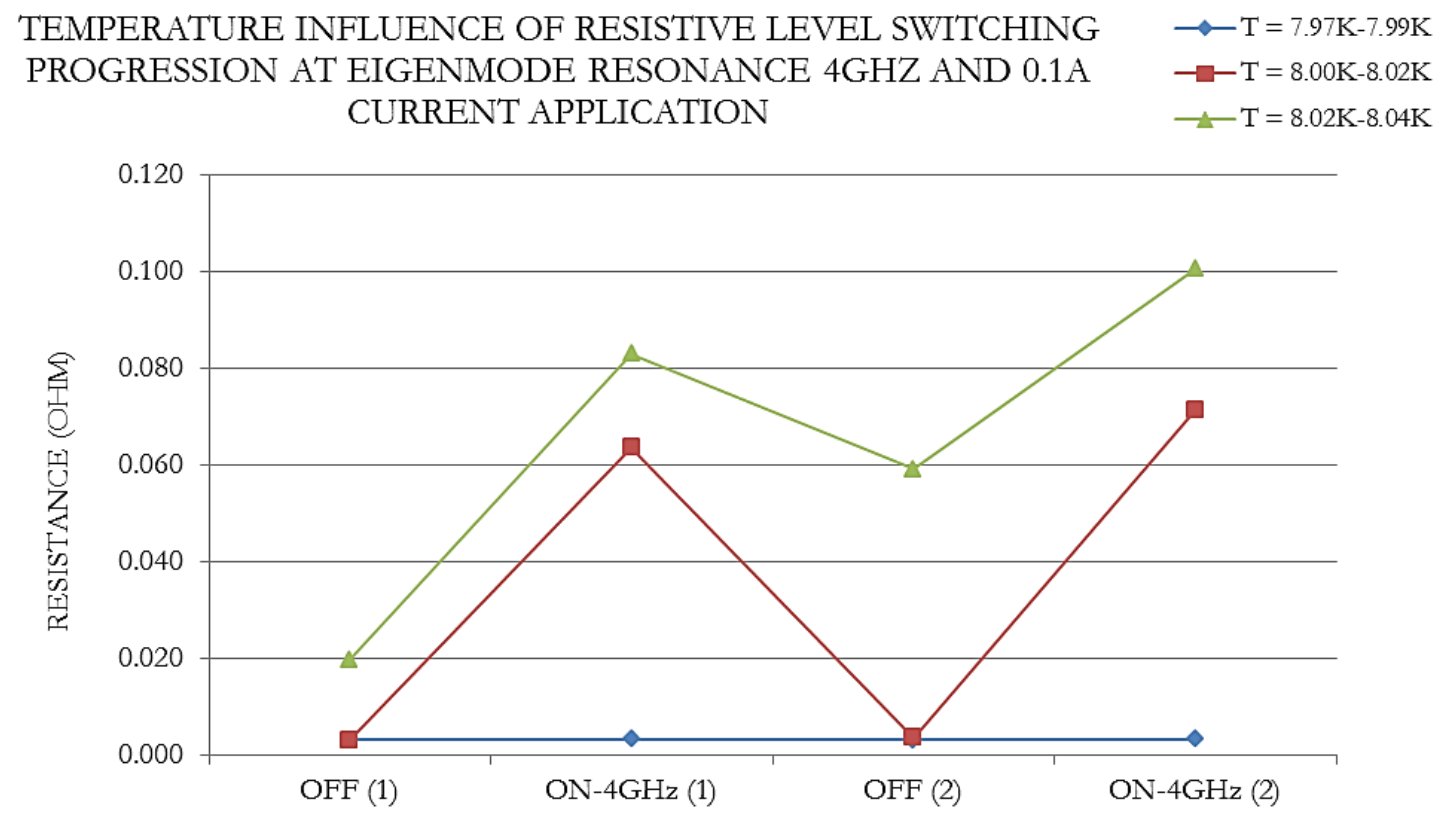

RF SWITCHING PROGRESSION STEPS

Figure 5.8: Temperature dependence of voltage level and reversibility at the dominant voltage developing frequency of $4 \mathrm{GHz}$ with a $7.28 \mathrm{~mm}$ total transmit monopole antenna extension.

Figure 5.7 depicts the high induced normalized resistance with RF switching steps at $4 G H z$; and Figure 5.8 shows the effect of temperature bias on the induced normalized resistance switch-off capability and thermal runaway. At very low temperatures $(<7.99 K)$, the device did not show any voltage development. At higher temperature ranges $(>8.02 K)$, the voltage development was not easily reversible. 


\subsubsection{Normalized Configuration Resistance Comparisons}

The following set of figures show the induced normalized resistance values for RF sweeps applied to several ACC configurations, as outlined in Table 4.2 .

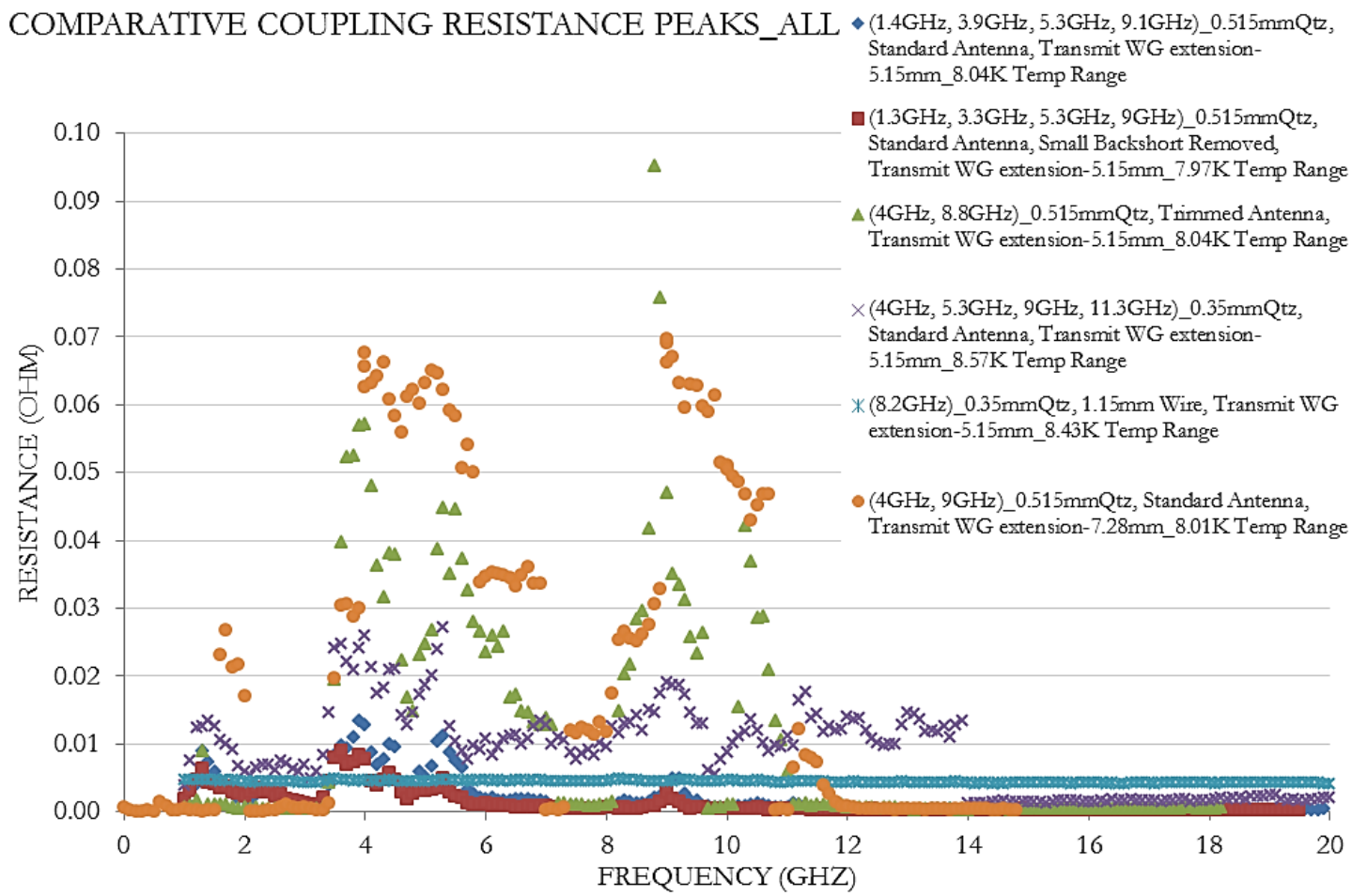

Figure 5.9: A comparison plot of all testing configurations showed deviations of scale in resistance levels caused by varied conditions. The two cases with highest resistance levels incorporated the $7.28 \mathrm{~mm}$ monopole extension and restricted transmission width, respectively.

To begin, all configurations are plotted together in Figure 5.9. Two of the sweeps produced especially high resistance levels. Emergent as the major contributors to these exceptionally high induced effects were the three testing factors of: $0.515 \mathrm{~mm}$ amorphous quartz dielectric, $7.28 \mathrm{~mm}$ coax extension and transmission width constriction. 


\subsubsection{Receive Antenna}

In Figure 5.10, a trimmed $\mathrm{Nb}$ transmission width in sample A-MS-TT allowed higher resistance values without alterations to the coupling profile. It functioned by mitigating $J_{S}$. The trimmed transmission width geometry did not present a dramatic contribution to resonance tuning, though higher resistance at $9 \mathrm{GHz}$ and an additional peak at $10.5 \mathrm{GHz}$ developed.

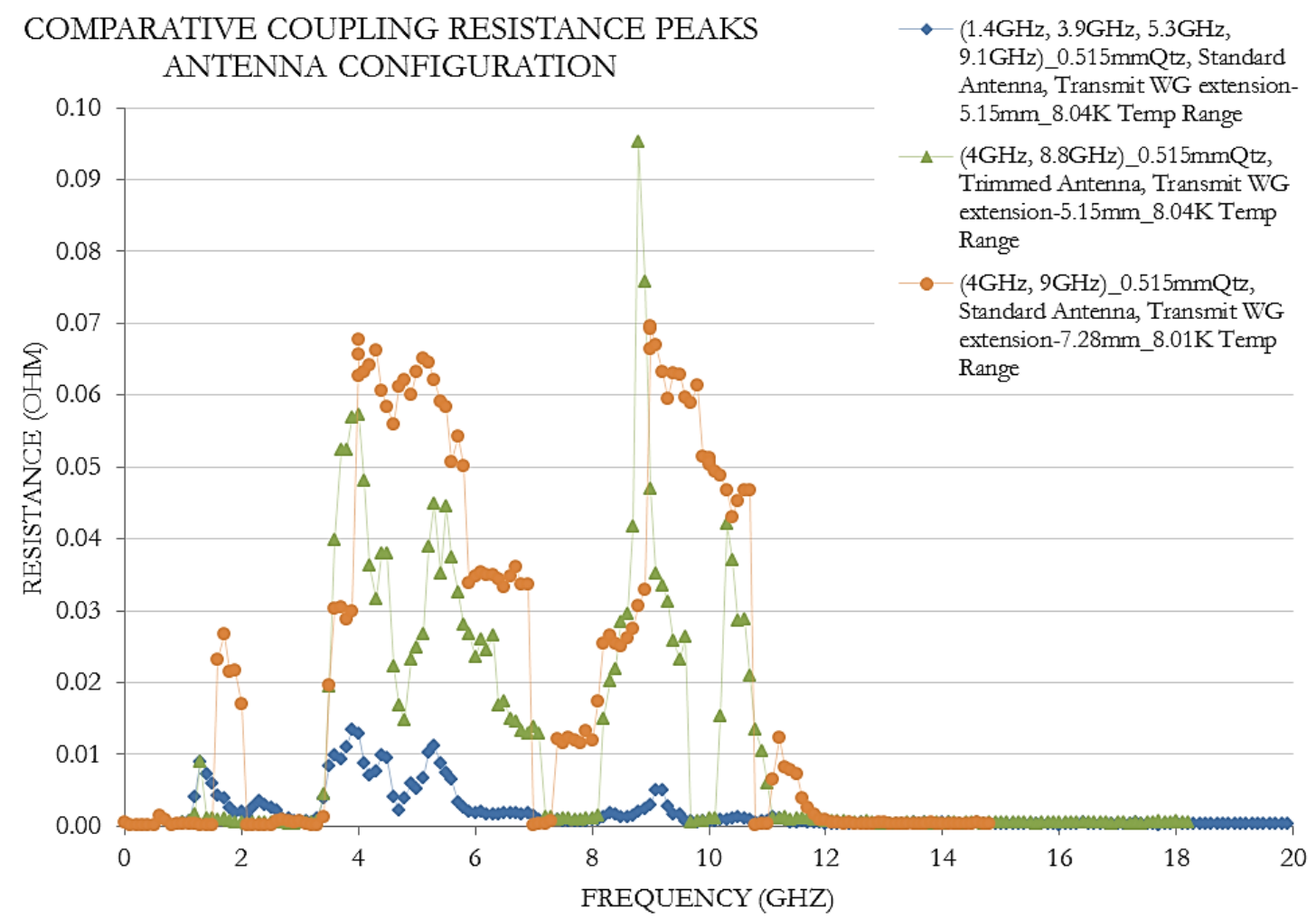

Figure 5.10: Comparative normalized resistance profiles for varied antenna configurations. Similar resistance levels were observed for trimmed $\mathrm{Nb}$ transmission width and the elongated monopole extension of $7.28 \mathrm{~mm}$. Comparison between the $7.28 \mathrm{~mm}$ monopole extension and the $5.15 \mathrm{~mm}$ monopole extension showed much higher relative resistance with the former.

Without transmission width constriction, the governing factor of resistance level in Figure 5.10 is the monopole transmit antenna extension. The $7.28 \mathrm{~mm}$ extension allowed 
peak resistances approximately an order of magnitude larger than those seen with a $5.15 \mathrm{~mm}$ monopole extension. This is particularly compelling because the $5.15 \mathrm{~mm}$ extension test was carried out at a higher temperature bias than the $7.28 \mathrm{~mm}$ extension.

\subsubsection{Quartz Dielectric}

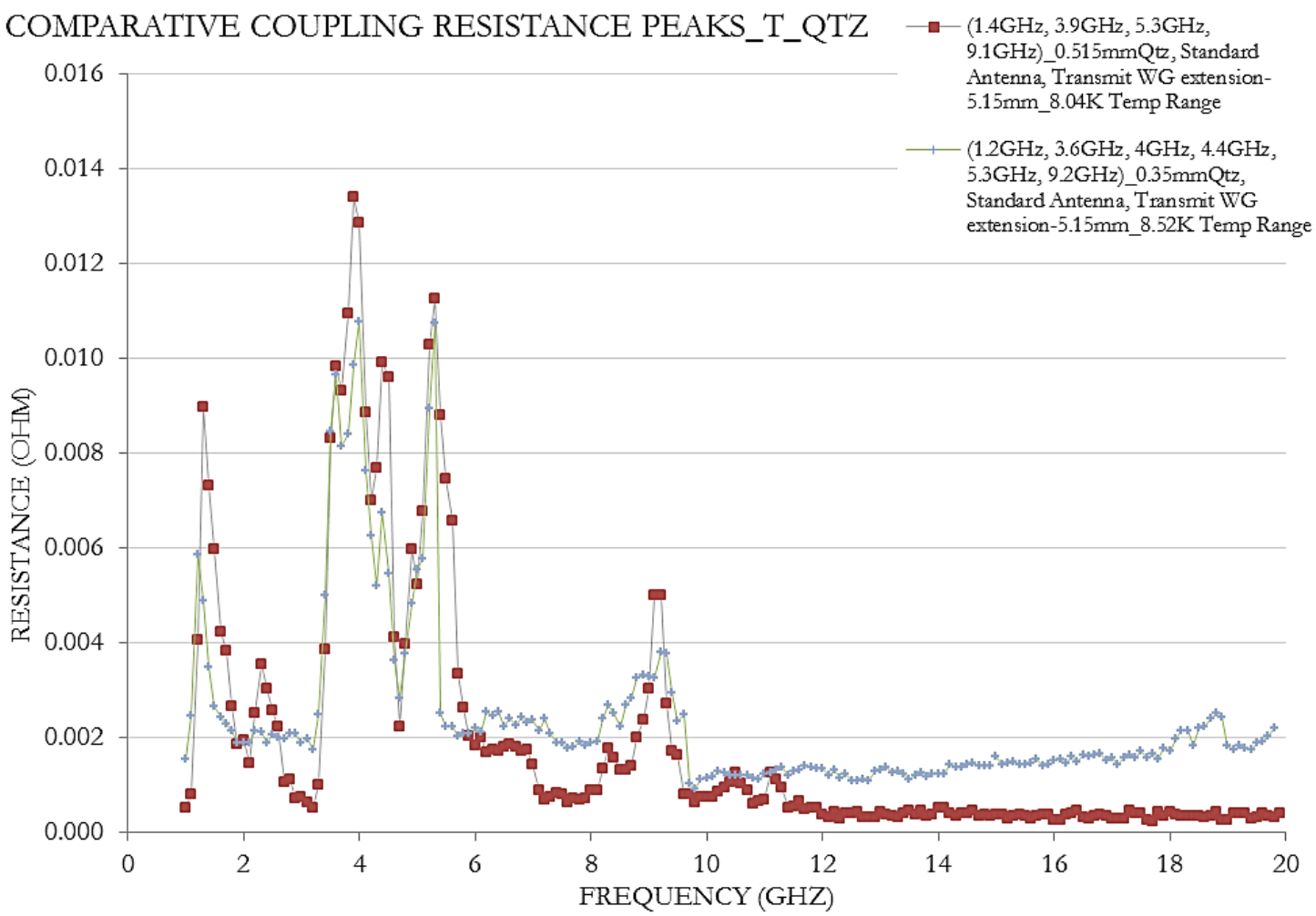

Figure 5.11: Identical testing parameters with variance only in the quartz showed similar coupling for the two quartz samples. The crystalline quartz displayed higher temperature stability than the amorphous sample.

The $0.515 \mathrm{~mm}$ amorphous quartz with standard antenna (A-MS-FT) favored $4 G H z$ and 9GHz; and $0.350 \mathrm{~mm}$ crystalline quartz with standard antenna (B-MS-FT) also favored $4 G H z$ and $9 G H z$, but with wider bandwidths and higher bias temperature. Notable of the crystalline quartz was that increases in the $\mathrm{Nb}$ normalized resistance happened in a gradual and controlled manner. The amorphous quartz sample showed more quick and drastic jumps 
in normalized resistance; which also entered thermal runaway with more ease than the crystalline-backed superconductor.

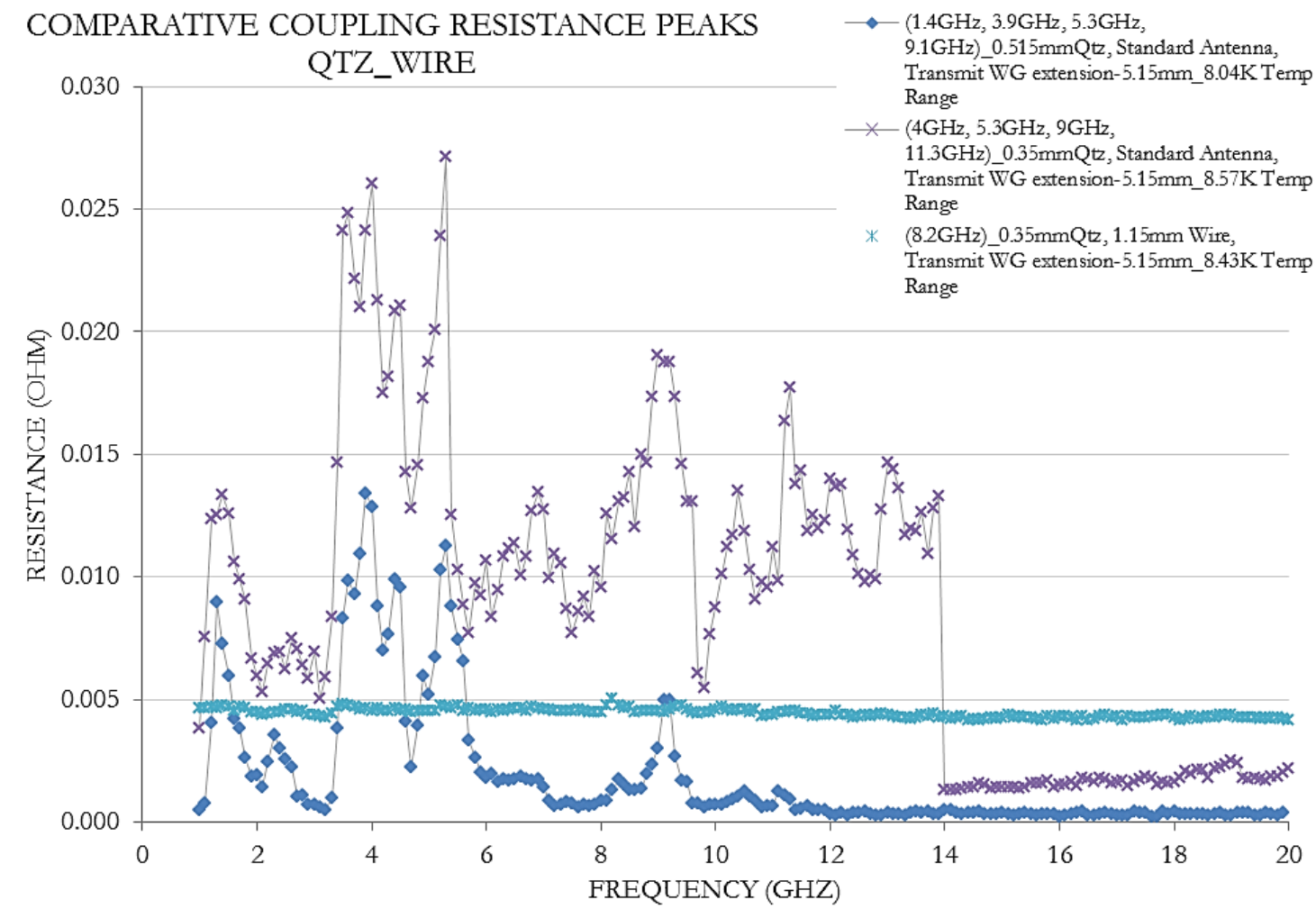

Figure 5.12: Measurements of a thin strip-line transmission geometry compared to $\mathrm{Nb}$ transmission surfaces incorporating the slot antenna show drastic deviation. The results indicate that the slot antenna is necessary for inducing a voltage within the superfluid.

The thin strip-line, as compared to the standard antenna, had only one slight coupling peak at $8.2 \mathrm{GHz}$. Comparative resistance profiles of standard receive slots on amorphous and crystalline quartz and the strip-line on crystalline quartz are displayed in Figure 5.12 , and confirm a higher temperature allowance before thermal runaway in crystalline quartz. Though induced voltage on the strip-line was not significant, the temperature profile showed more pronounced activity. This will not be discussed in the context of the ACC; Appendix F contains compelling results from swept current tests on the strip-line. 


\subsubsection{Altered Waveguide}

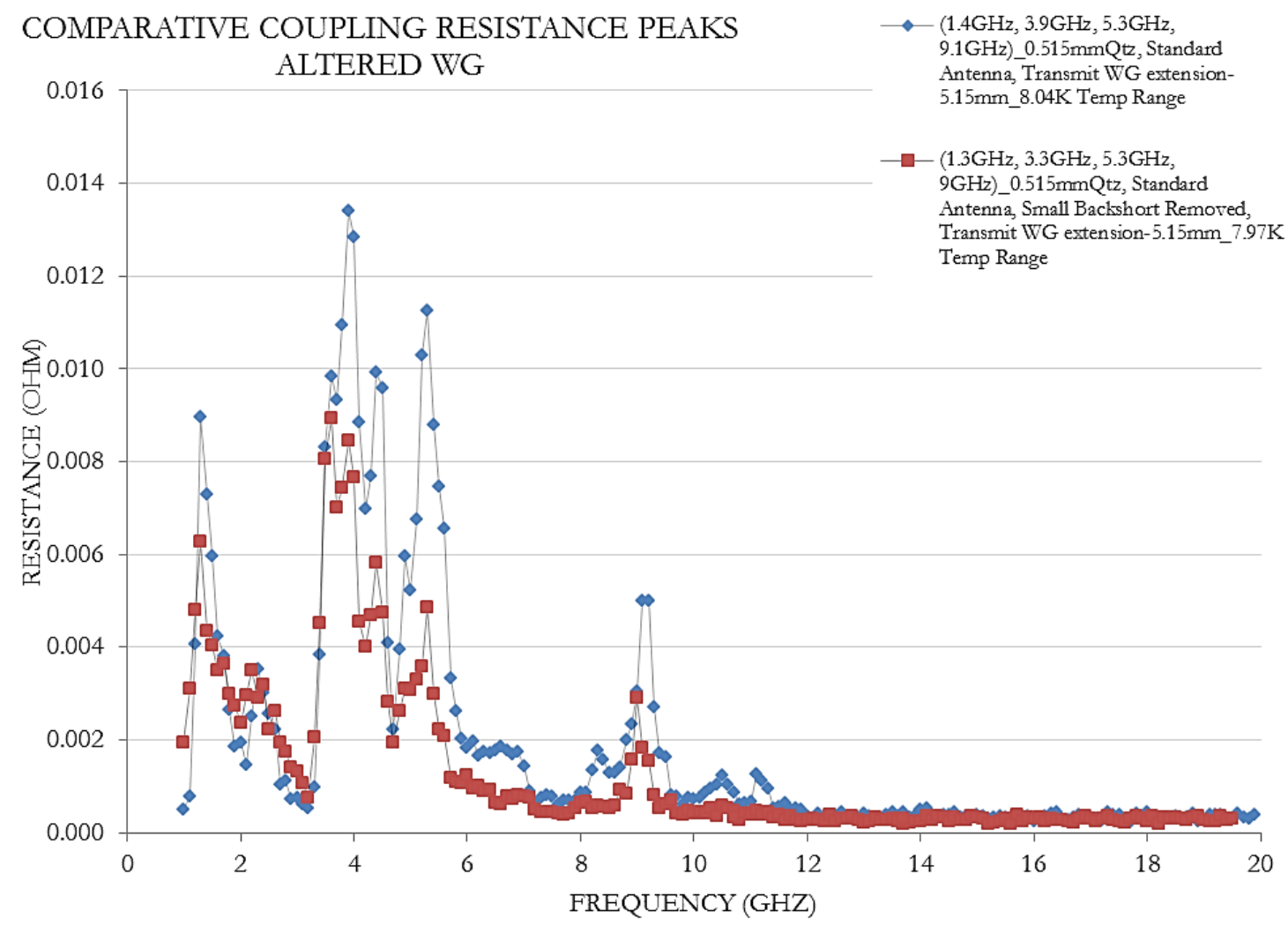

Figure 5.13: The removal of BS1 during testing, with all other variables held constant, did not show significant changes in normalized resistance levels. A small reduction in resistance without BS1 is likely a product of direct exposure of the quartz and transmit monopole to LHe, lowering the thermodynamic energy in the slug.

From Figures 5.13 and 5.14, the combination of BS1 and the $0.515 \mathrm{~mm}$ quartz slug support the $4 G H z$ range, and $\mathrm{BS} 2$ supports $9 G H z$. Though, the $4 G H z$ range is closely linked to the slug, since it exists in all waveguide alteration scenarios. With BS2 removed, the resistance and temperature in the $4 G H z$ range increased, indicating that $\mathrm{BS} 2$ was likely damping $4 G H z$, and also sinking thermal energy from the $\mathrm{Nb}$. 


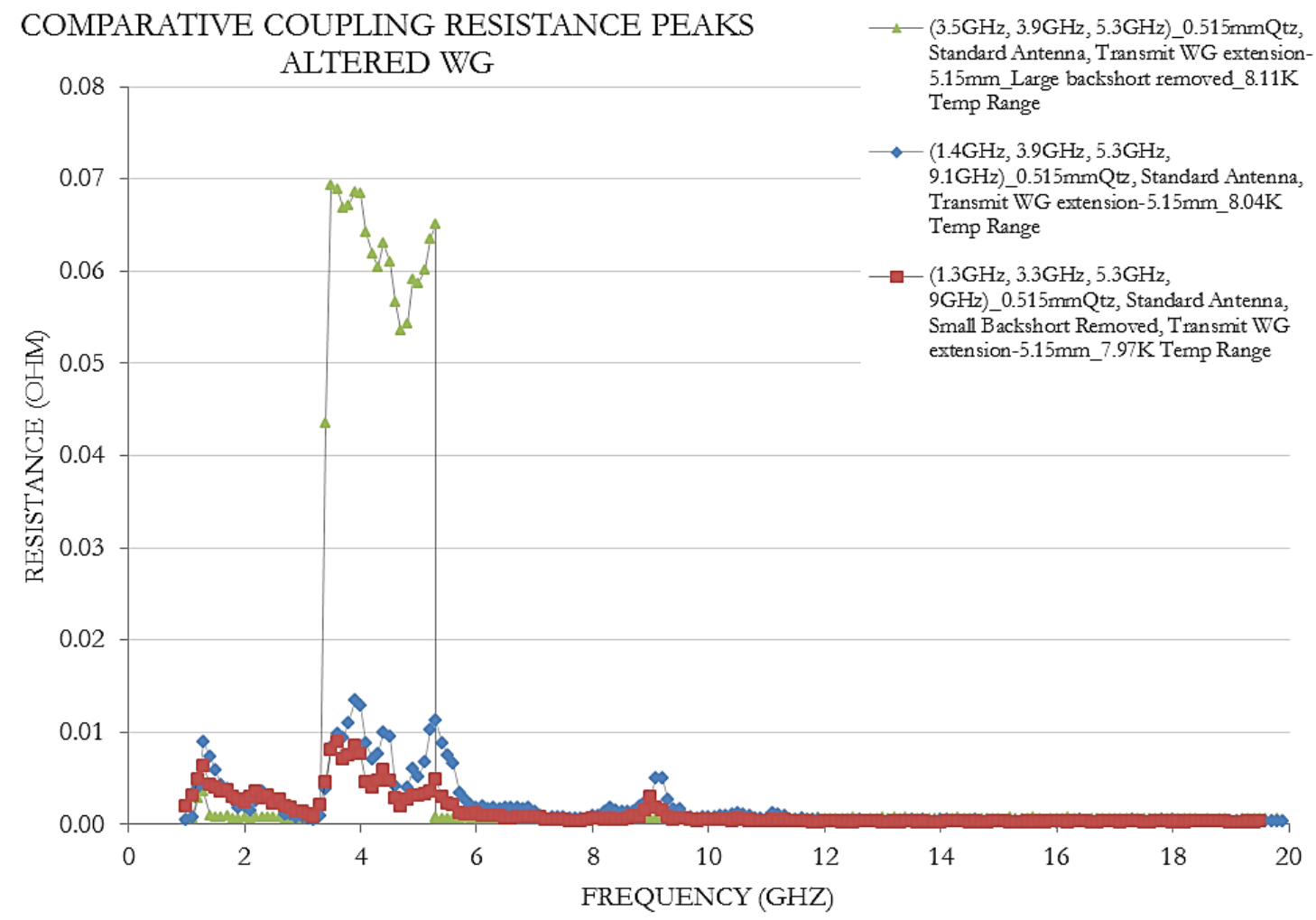

Figure 5.14: The removal of BS2 during testing, with all other variables held constant, showed a significant increase of normalized resistance at $4 G H z$; and also a notable reduction in resistance at $9 \mathrm{GHz}$. 


\subsubsection{Coaxial Transmit Extension}

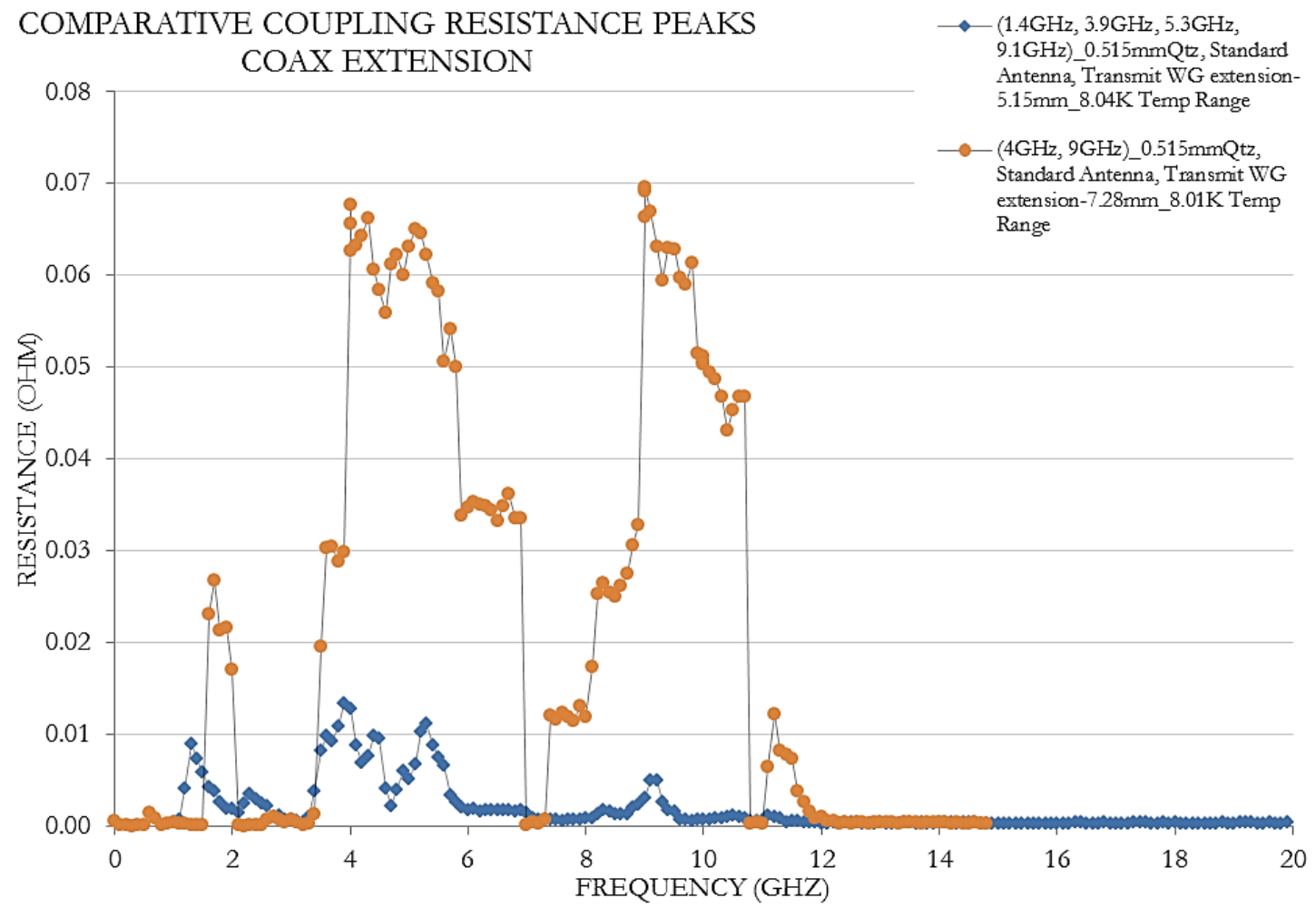

Figure 5.15: Focused representation of discrepancies in normalized resistance profile, only in reference to transmit monopole extension.

A narrowed scope view of monopole lengths shows the total coax extensions of $5.15 \mathrm{~mm}$ and $7.28 \mathrm{~mm}$ have similar profiles, but $7.28 \mathrm{~mm}$ carries higher induced values and wider bandwidths. $7.28 \mathrm{~mm}$ also allows a small spike at $11.5 \mathrm{GHz}$, higher than the range of $5.15 \mathrm{~mm}$. The $3.14 \mathrm{~mm}$ extension required more current $(0.17 \mathrm{~A})$ for the same voltage level $(0.1 \mathrm{~V})$ as $7.28 \mathrm{~mm}$ extension $(0.1 \mathrm{~A})$. The $3.14 \mathrm{~mm}$ extension also required $\sim$ seconds before fully developing a voltage, while the $7.28 \mathrm{~mm}$ extension developed in $\mathrm{ms}$ or less (depending on temperature). 


\subsubsection{Frequency-Induced Voltage and Temperature}

The following set of figures are shown to illustrate nuances in the testing process. Comparison between Figures 5.16 and 5.18 were made to assess the affect of free energy momentum on the perceived induced voltage at each frequency level. Swept frequencies created profiles which indicated a wider array of voltage-inducing frequencies than was shown in isolated frequency applications, where the momentum from one frequency could not carry over to the next sequentially swept frequency level. The isolated frequency applications established $4 G H z$ and $9 G H z$ as the dominant frequencies of interest for inducing voltages in the $\mathrm{Nb}$.

VOLTAGE VS. FREQUENCY - ISOLATED SAMPLES (NOT SWEPT)

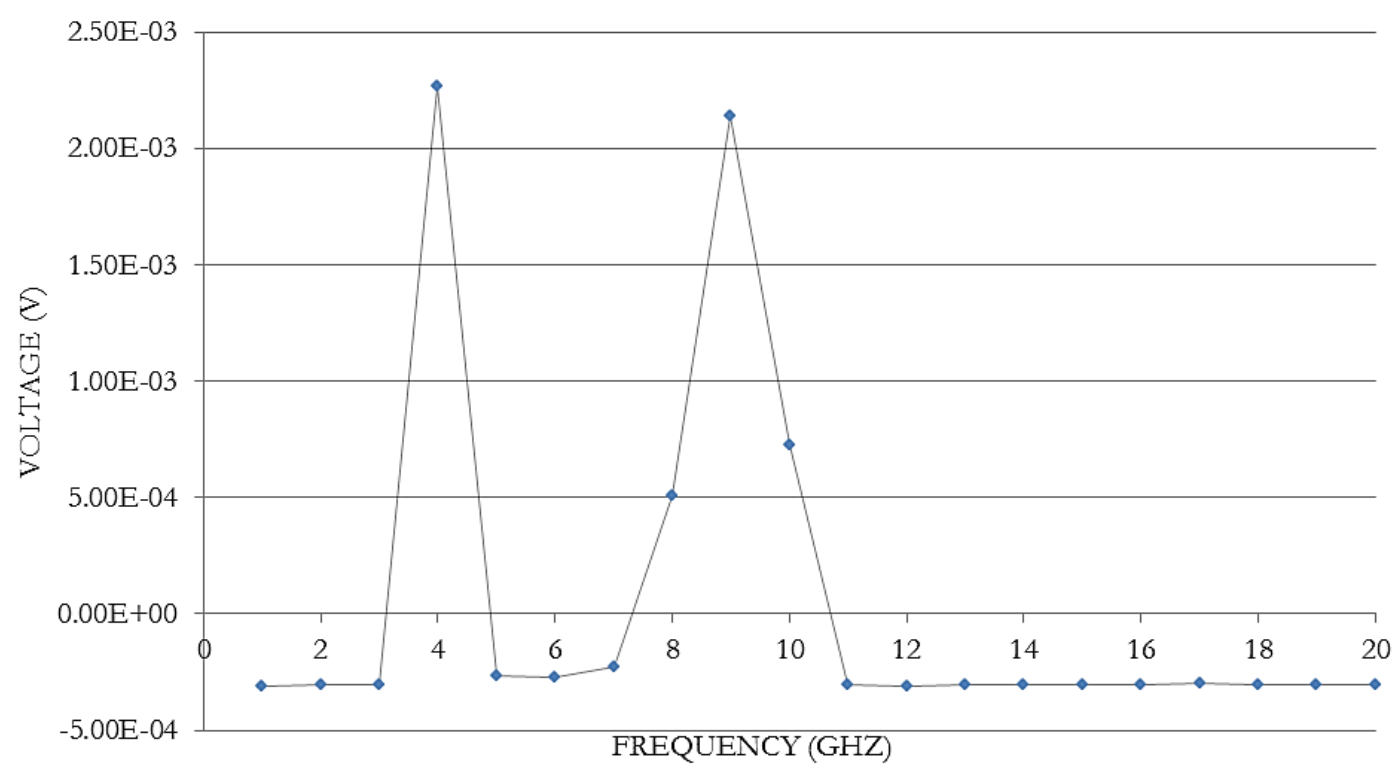

Figure 5.16: Isolated frequency applications with a $7.28 \mathrm{~mm}$ monopole transmit antenna extension. Values were calculated from an average of 11 samples taken over a $\sim 5 s$ total time interval. 


\section{TEMPERATURE VS. FREQUENCY - ISOLATED SAMPLES}

(NOT SWEPT)

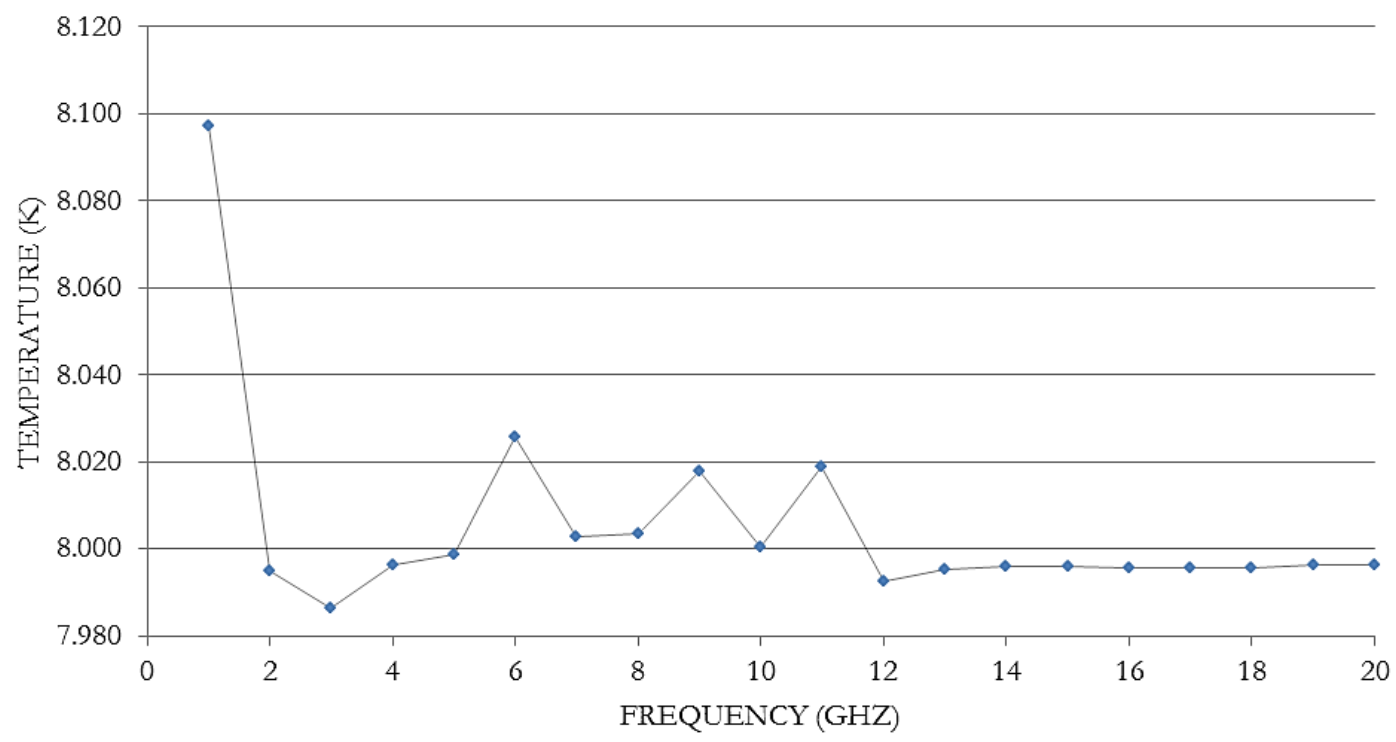

Figure 5.17: Temperature tracking correspondent with isolated frequency applications at a $7.28 \mathrm{~mm}$ monopole transmit antenna extension. Values were calculated from an average of 11 samples taken over a $\sim 5 s$ total time interval.

Examination of the low voltage values from Figures 5.16 and 5.18 shows that the lowest voltage levels are below $0 \mathrm{~V}$. The voltage offset with applied current to the $\mathrm{Nb}$ transmission line was approximately $-300 \mu \mathrm{V}$, whether or not RF radiation was present. This was attributed to Andreev reflections at the $\mathrm{Au}-\mathrm{Nb}$ interface, with a transport current across the junction of the normal-superconducting boundary. This happens as a result of differing Fermi velocities between the metals, and occupied quasi-particle energy states in the superconductor.[49] These cause a fraction of the electrons at the interface to reflect back into the normal metal as holes. Thus a small negative charge disequilibrium is formed.[49]

Figures 5.17 and 5.19 show tracked temperature profiles corresponding to the isolated and swept frequency sampling intervals, respectively. Notably, two major temperature 
spikes occurred in the $1 G H z-2 G H z$ range. This indicates a thermodynamic disturbance, which may have an effect on the small voltage peak just before $2 G H z$, shown in Figure 5.18. Excluding the major temperature outliers, Figure 5.20 shows small temperature peaks occurring in the $6 G H z$ and $9 G H z-12 G H z$ range as well.

VOLTAGE VS. FREQUENCY 1-15GHZ SWEEP; 100MHZ RESOLUTION

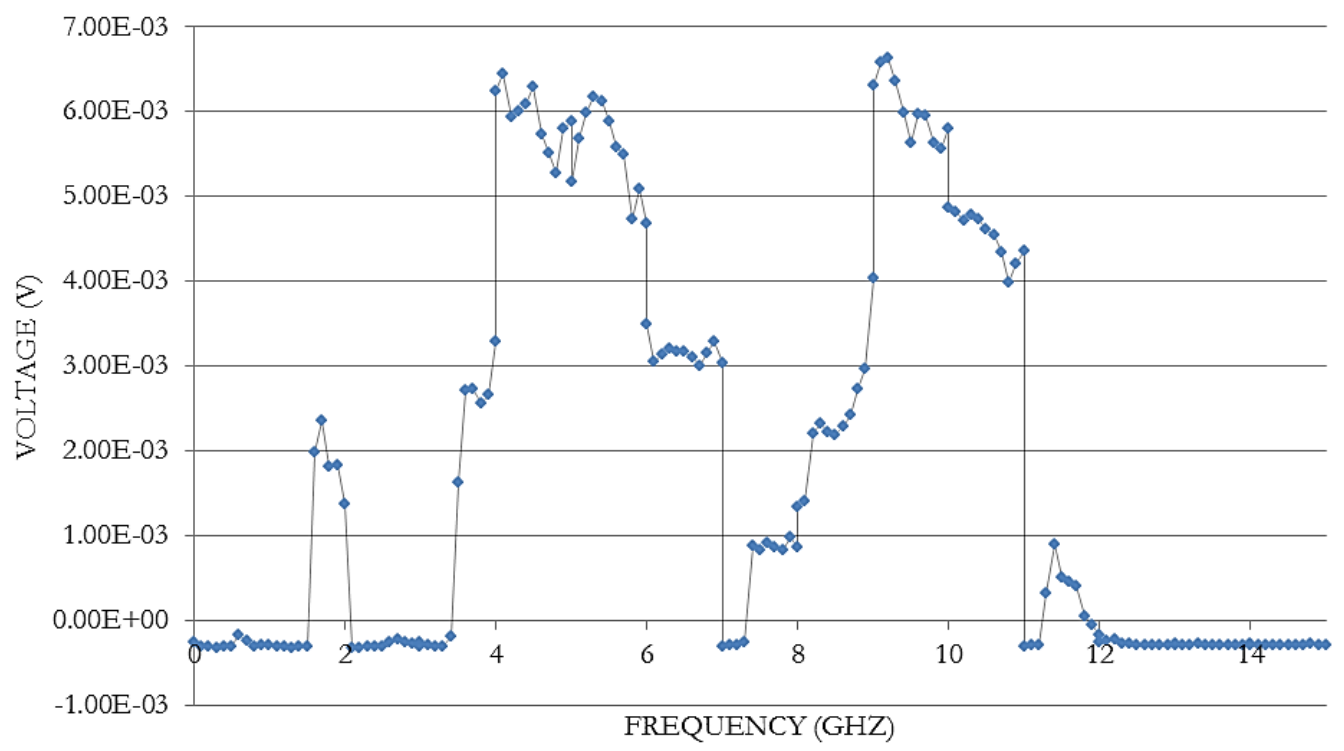

Figure 5.18: Induced voltage over sequentially swept frequencies, with $100 \mathrm{MHz}$ resolution, at $7.28 \mathrm{~mm}$ monopole transmit antenna extension. Samples were taken as single datum, at a rate of 1 sample per $100 \mathrm{~ms}$.

Figures 5.16 through 5.20 indicate effects in terms of both electric and thermodynamic processes happening within the $\mathrm{Nb}$ due to applied $\mathrm{RF}$. 
TEMPERATURE VS. FREQUENCY 1-15GHZ SWEEP; 100MHZ

RESOLUTION

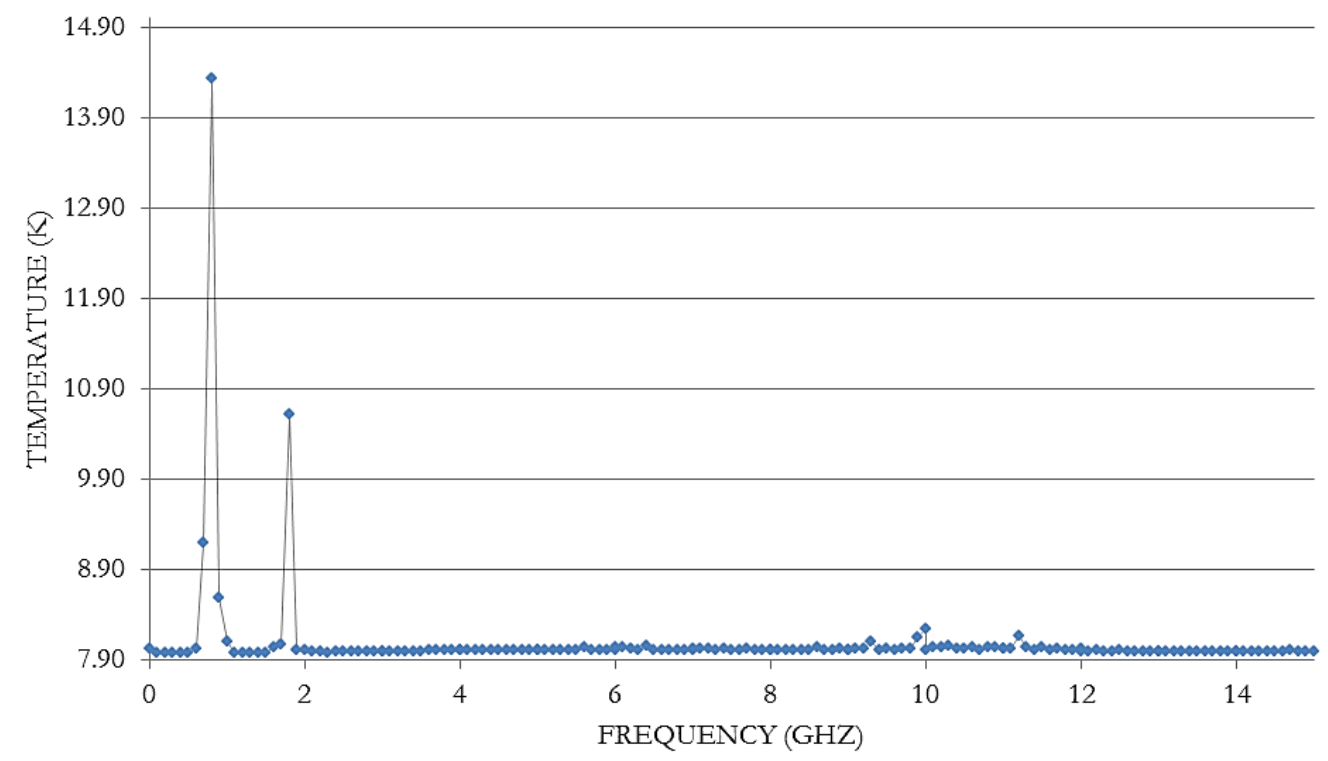

Figure 5.19: Temperature tracking correspondent with sequentially swept frequencies, with $100 \mathrm{MHz}$ resolution, at $7.28 \mathrm{~mm}$ monopole transmit antenna extension. Samples were taken as single datum, at a rate of 1 sample per $100 \mathrm{~ms}$.

TEMPERATURE OMIT OUTLIERS 3-15GHZ SWEEP; 100MHZ RESOLUTION

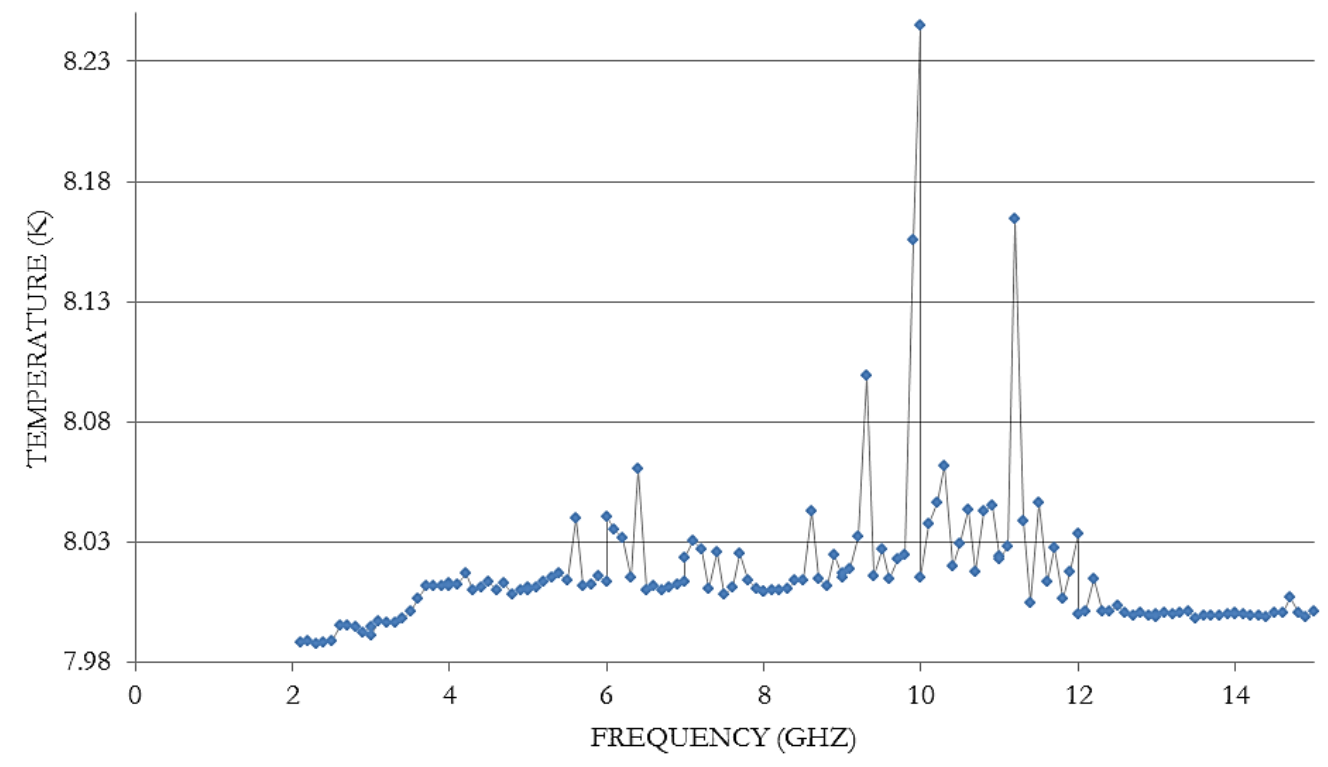

Figure 5.20: Outlier exclusion of Figure 5.19 revealed frequency-induced thermodynamic changes in the $6 G H z$ and $9 G H z-12 G H z$ range. 


\subsubsection{Shunt Path for Latched Switching}

Tests incorporating temperature bias near $T_{C}$ ran the risk of thermal runaway to a latched normal state at current levels above $\sim 0.3 I_{C}$. A thermal runaway event under these conditions is exemplified in Figure 5.21. In effort to utilize this effect, a shunt resistance path was applied to the input current terminals of the source which fed the ACC. A line with very small resistance was predicted to conduct upon a normal transition of the $\mathrm{Nb}$, which would render the ACC at a larger resistance value than the shunt line. Figure 5.22 shows the basic shunt circuit schematic, with the resistive load located outside of the cryogenic environment.
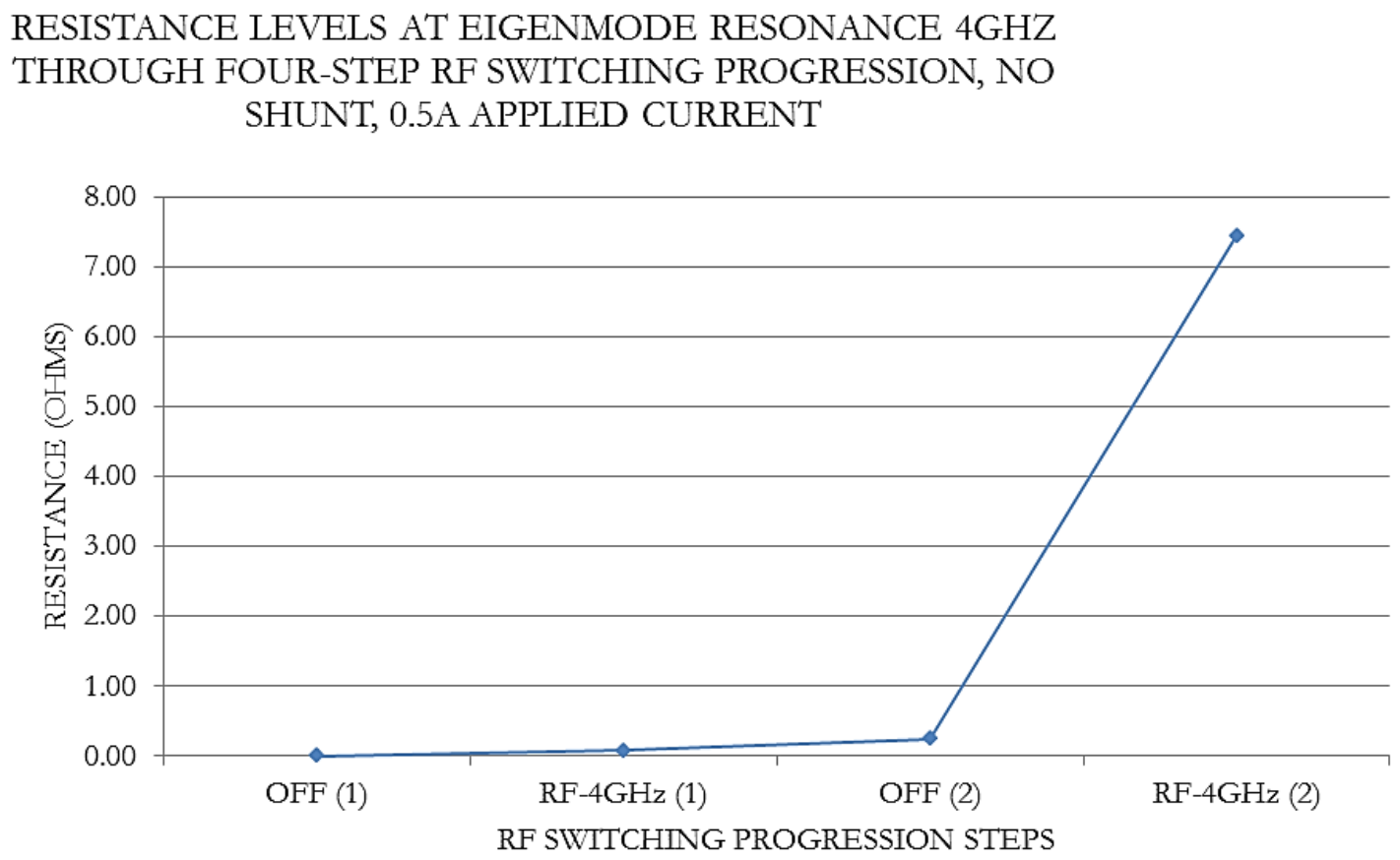

Figure 5.21: Excessively high energy levels within the ACC created thermal runaway, which caused latched switching events. 


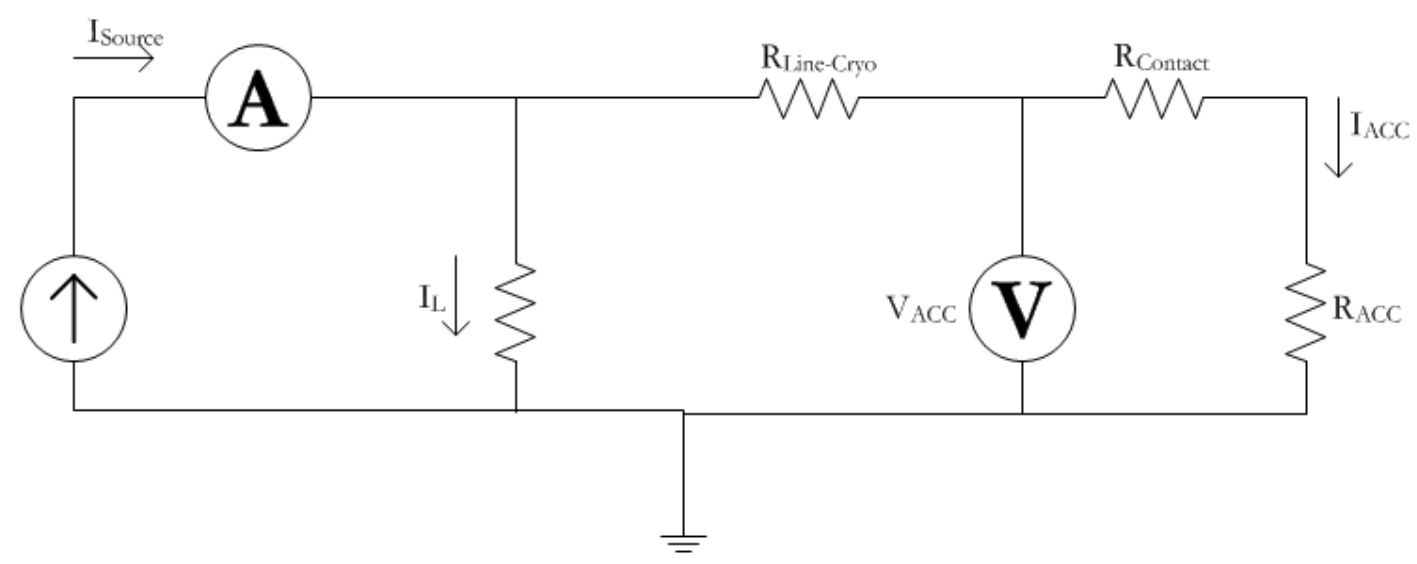

Figure 5.22: Equivalent Circuit for shunt load testing configuration.

$$
I_{A C C}=\frac{R_{L}}{R_{C}+\left(R_{l}+R_{c o}+R_{A C C}\right)} I_{\text {Source }}
$$

The shunt resistance acted as a current division path to maintain stability in the ACC, rather than as a full current transfer path. Here, the ACC never fully transitioned through thermal runaway, thus the parallel lines maintained similar resistance levels to each other. A repeat test, under the same conditions as those producing the results in Figure 5.21, was run with a small resistive shunt path and gave the results in Figure 5.23. An element of irreversibly increased free energy was apparent in the $\mathrm{OFF}(2)$ datum, but thermal runaway was averted completely with use of a shunt path. Though the temperature was beyond a controllable point, the current through the ACC was limited because of the shunt path, which caused overall energy stability.

In order to create a true shunt path to redirect current from a latched ACC normal transition, a capacitor or energy threshold switch would be required in place of a simple resistor. Results from tests, run at several shunt resistance levels, are shown in Table 5.1. 


\section{RESISTANCE LEVELS AT EIGENMODE RESONANCE 4GHZ THROUGH FOUR-STEP RF SWITCHING PROGRESSION, 0.5 OHM SHUNT AND 0.5A APPLIED CURRENT}

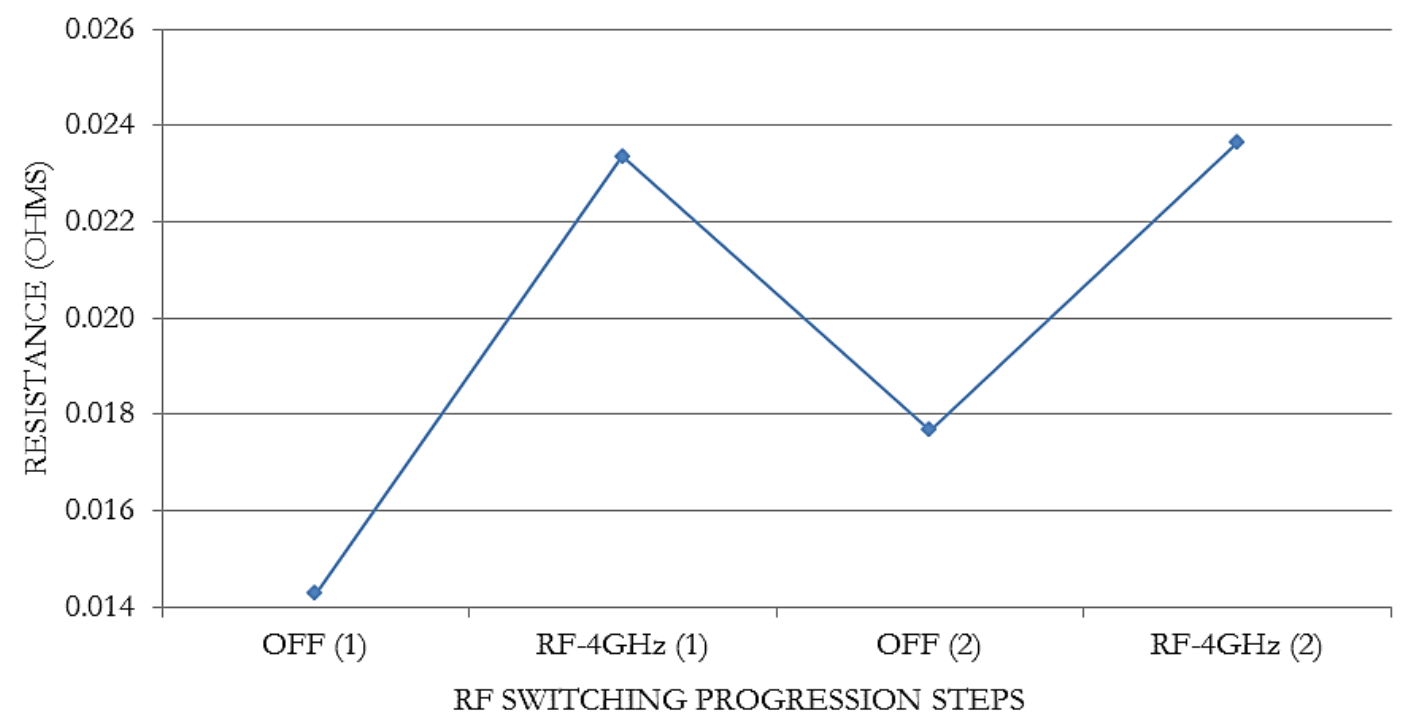

Figure 5.23: Shunt-impedance-controlled RF switching at an excessively high energy level maintained relative stability.

Significant values correspond with variables shown in Figure 5.22. They were calculated with metered readings and the mathematical model of equation 5.1. The shunt line resistance needed to be below a threshold value to allow voltage switching with RF. Otherwise, allowed current levels between the lines at higher resistance caused excessive energy within the system. 


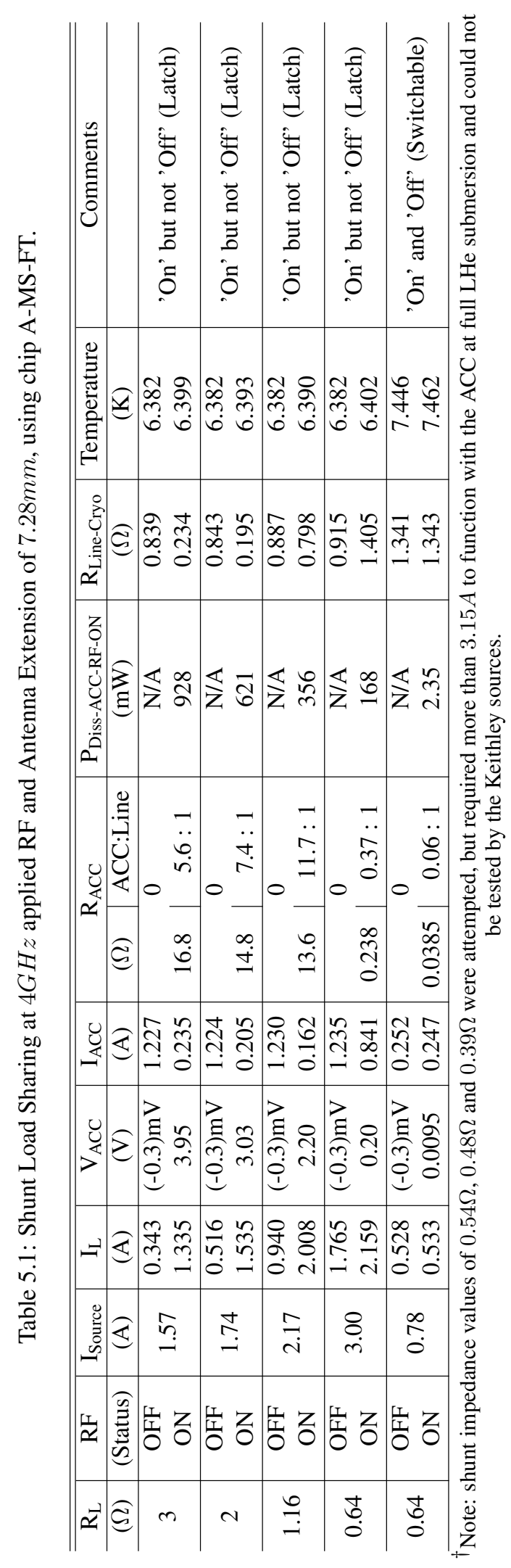




\subsubsection{Swept Power Level}

Swept power levels over the range of $1 d B m-30 d B m$ are shown in Figure 5.24 for comparative testing scenarios.

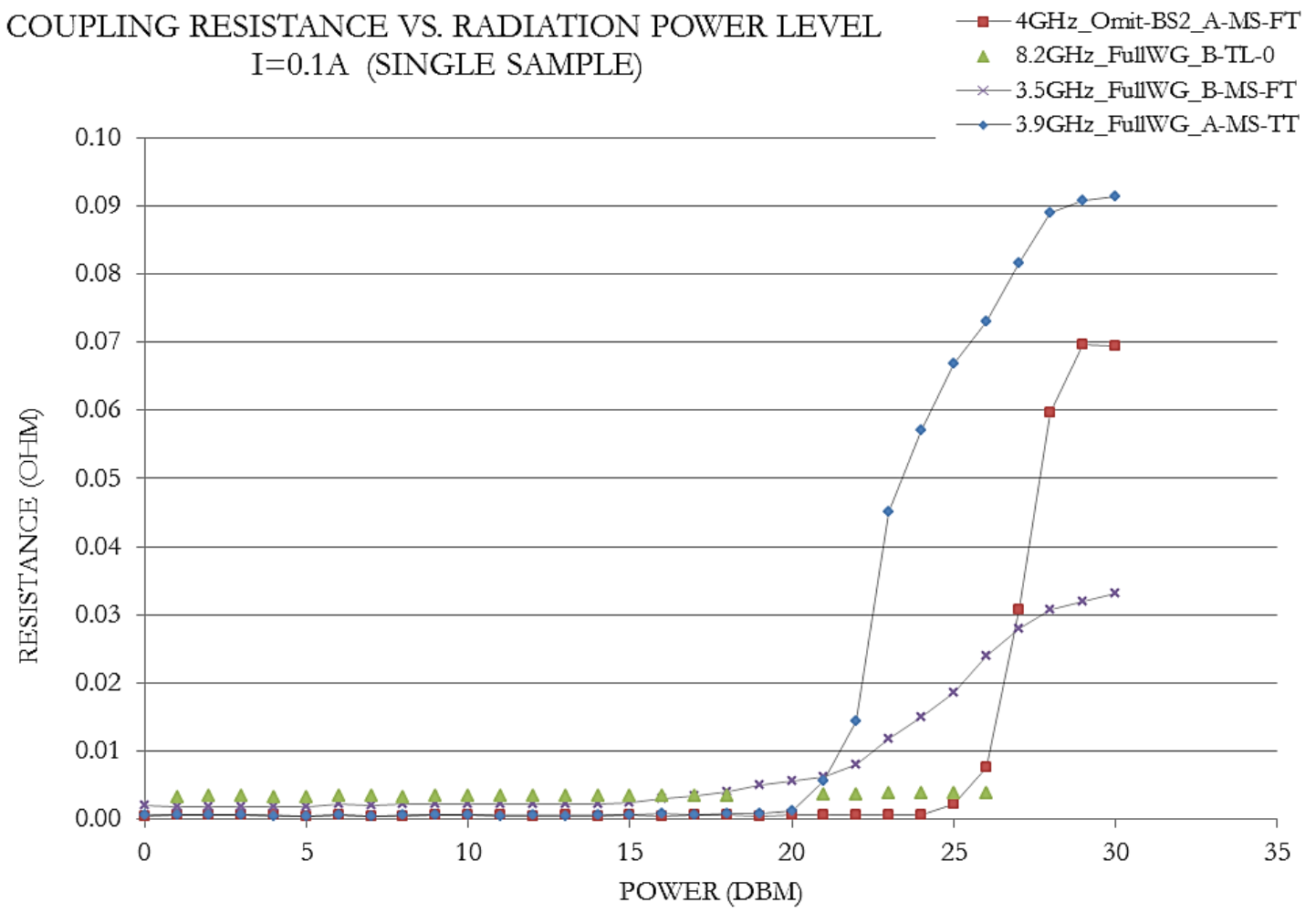

Figure 5.24: Normalized coupling resistance for swept power levels, comparatively applied to four separate testing scenarios.

The omission of BS2 for chip A-MS-FT illustrated a higher input power level requirement for transition, as well as a lower induced normalized resistance upon transition. With the full waveguide intact and a deviation between quartz chips, in A-MS-FT and B-MS-FT comparison, the slow and controlled rising voltage was again noticed for crystalline quartz. A-MS-FT and B-MS-FT, in these tests, initiated a rise in normalized resistance nearly simultaneously in Figure 5.24, but A-MS-FT displayed a jump, while B-MS-FT rose slowly 
and settled at a lower comparative resistance level. The strip-line, without a slot antenna, B-TL-0, showed insignificant comparative resistance levels when held against other samples. For all tests, the power level threshold to induce noticeable voltage was above $20 \mathrm{dBm}$. Slight deviations in applied frequency, combined with quartz type, influenced the necessary power level to cause voltage development. 


\section{Chapter 6: Discussion}

Major findings from tested samples were interpreted in terms of the project goal: establishment of a superconducting-to-normal transition with applicability to a switching actuation. Nuances of coupled temperature alterations and material interfacing effects were left as observed results, as they contributed only to future design considerations. The following sections entail the voltage-inducing quantum phenomenon at the design frequency; as well as at the lower frequencies which enacted evanescent modes.

\subsection{Post-Testing HFSS Model}

Adjustments were made to the HFSS model, accounting for: evanescent mode operation, full chip dimensions, cryogenic conditions, varied monopole extensions, and deviations in $\mathrm{Nb}$ sheet impedance.

Figure 6.1 shows the longitudinal electric wave propagation at $4 G H z$ at a maximum strength of $\sim 0.5 \mathrm{~V} / \mathrm{mm}$. Apparent in this illustration is that the longitudinal electric wave is not supported within the waveguide at $4 G H z$. However, the distance of evanescent mode propagation before full attenuation did reach into the dielectric.

The close positioning of the two antennae across the width of the quartz chip allowed evanescent modes to effect the receive slot before they were fully attenuated within the 


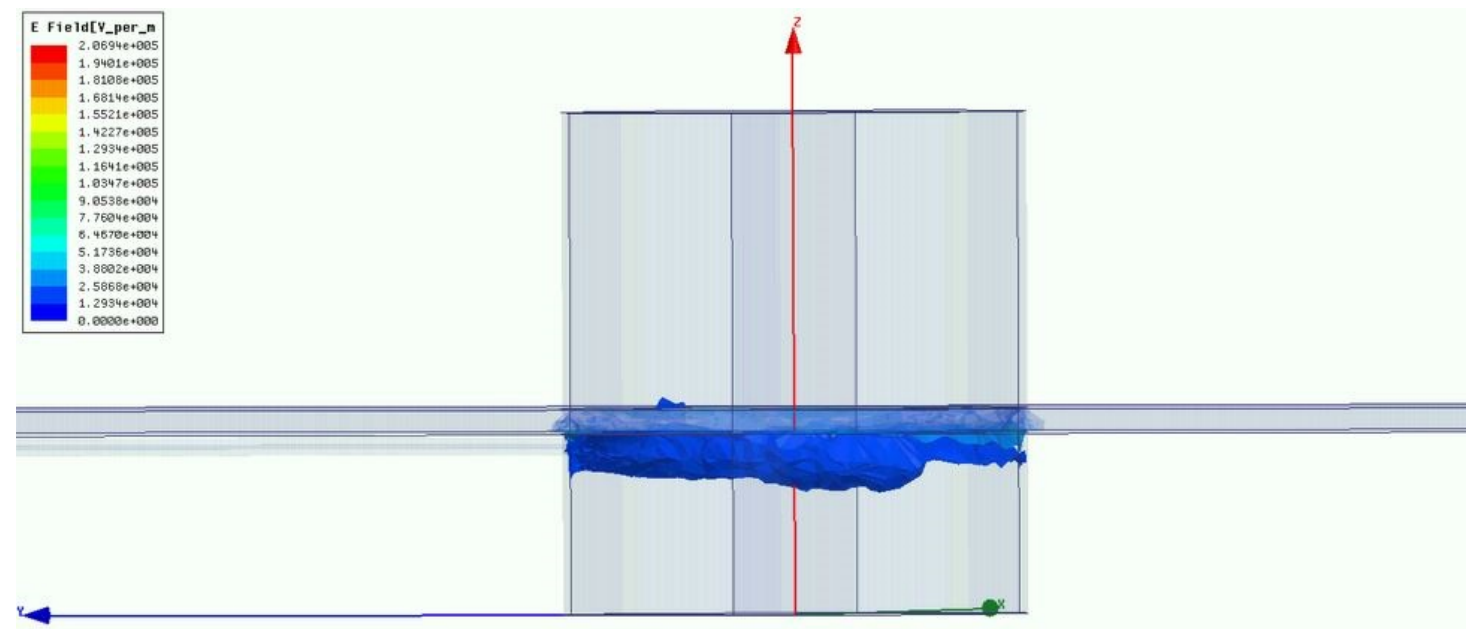

Figure 6.1: Post testing HFSS device maximum longitudinal E-field analysis at $4 \mathrm{GHz}$. A travelling field was expected along the z-axis for the waveguide to support any given frequency; which did not occur at $4 G H z$.

waveguide. Analysis of the magnetic field within the device, Figure 6.2, evidenced a perpendicular $\mathrm{H}$-field component subject to the $\mathrm{Nb}$ film, with a maximum strength of $\sim 18 \mathrm{~mA} / \mathrm{mm}$. The two lobes of the perpendicular H-field were positioned directly on the transmission surfaces around the slot antenna.

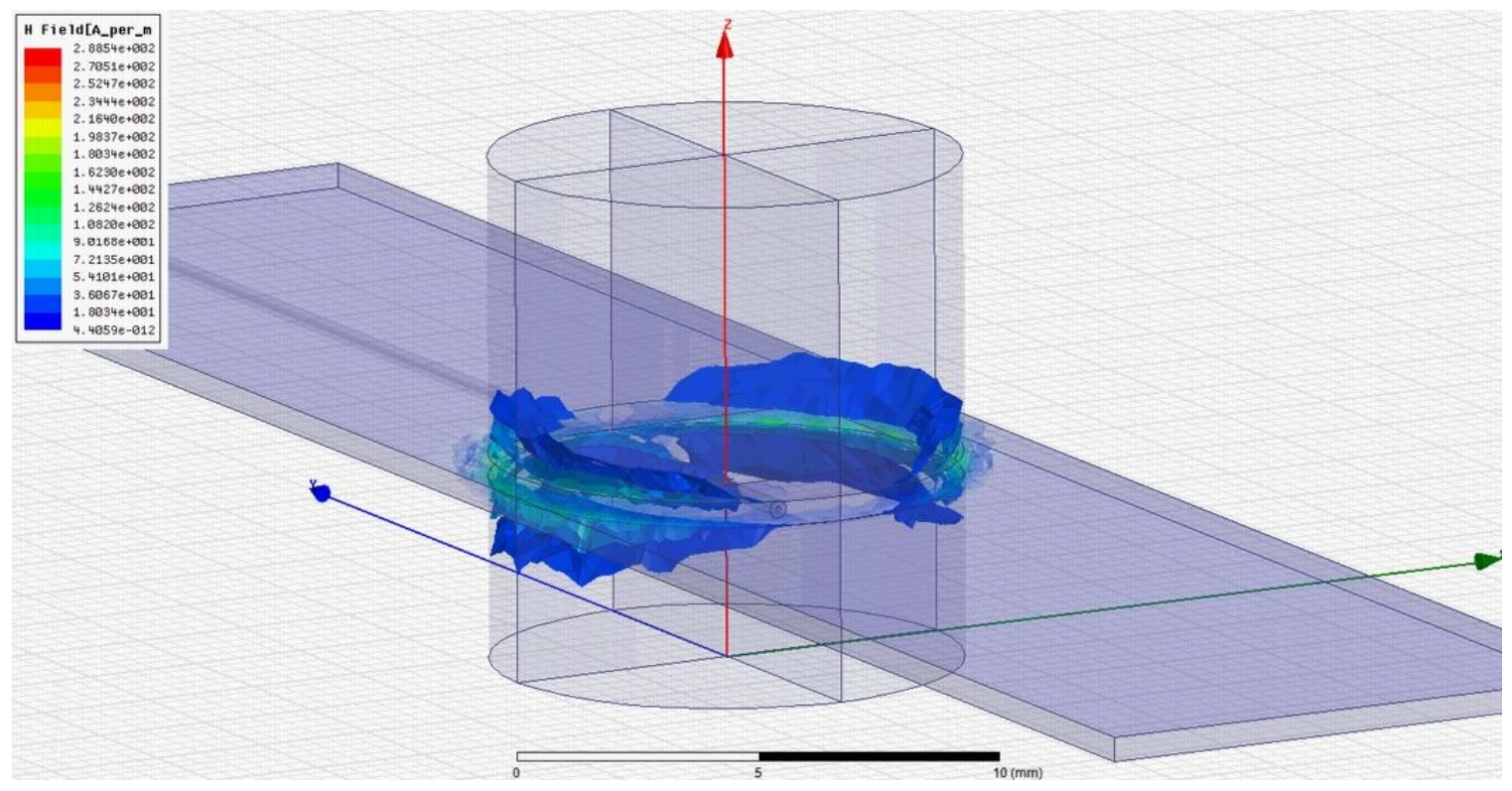

Figure 6.2: Post testing HFSS device maximum H-field analysis at $4 G H z$. A null field at the quartz was expected for the device to function as designed. Rather, the quartz supported a perpendicular $\mathrm{H}$-field at $4 G H z$. 


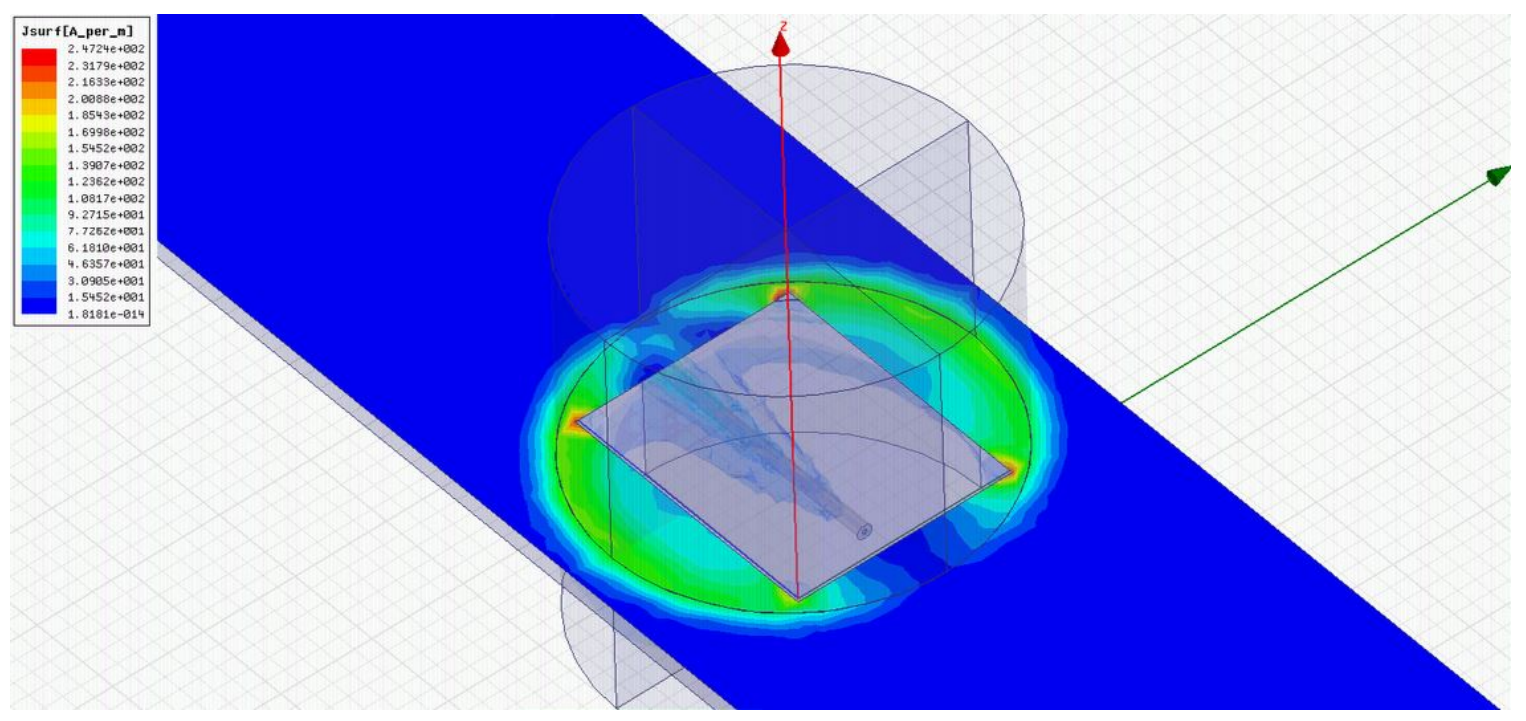

Figure 6.3: Post testing HFSS device maximum surface current density at $4 G H z$, with reduced sheet resistance to account coupling into a superfluid.

Coupling on the slot antenna under altered functionality at $4 \mathrm{GHz}$ and sheet impedance of $3 \mathrm{~m} \Omega$, Figure 6.3 , gave a maximum induced surface current density of $\sim 25 \mathrm{~mA} / \mathrm{mm}$.

\subsection{Hypotheses for Results}

The coupling mechanism of highest interest, with reversible induced voltages at $4 G H z$ and $9 G H z$ peaks was attributed to slot-line induced development of an Abrikosov vortex within the thin film. [7, 8, 23, 49] The qualifying electromagnetic field condition for an Abrikosov vortex state in a thin film superconductor is a strong magnetic field perpendicular to the film surface.[23, 49]

When the waveguide functioned at the design frequency, the longitudinal magnetic field component was assumed effectively null at the chip surface, with a high lateral electric field.[3, 41] Therefore, effects above the cutoff frequency of the waveguide were mainly attributable to quasi-particle redistribution. Further corroboration with this assertion was 
provided by the induced voltage profile at the design frequency, which followed an expected quasi-particle energy state elevation trend.[49]

\subsection{Waveguide-Induced Quasi-Particle Redistribution}

Voltage development from energy state elevation of quasi-particles was expected to occur in a smooth upward transition, rather than a sudden jump. The observed voltage development was slight, likely due to the fact that the applied RF energy was at the low end of the disruption bandwidth. The transition was also irreversible within the utilized testing conditions; further investigations on consistence and potential use of this property are needed.

The enhanced voltage effect required specific current levels $(\geqslant 1.47 A)$ and temperature profiles.[20, 49] In the case where $1.445 A$ was applied during testing, voltage development seemed to be suppressed with RF radiation, indicating gap enhancement. This may be attributable to human error in the timing of RF application. At time $=0$ in the temporal tests, current and RF radiation were applied simultaneously with push-buttons, rather than automated processes. The typical human reaction time is $\sim 100 \mathrm{~ms}$.[15] Though the voltage suppression phenomenon was repeatable, the error margin on the time scale due to human response deviations in push-button actuation is sufficiently large to render the results unreliable.

Considering the possibility that voltage suppression was occurring through quasi-particle redistribution, it was a product of gap enhancement. The energy gap of a superconductor can be widened, strengthening the superconductive state, through exposure to microwave 
signals with photon energy below that of the cooper-pair breaking energy gap, when the energy state of the material is within a specific range.[49] The process happens as a result of elevating quasi-particles to an energy level $E_{k}$, where $\Delta \leqslant E_{k} \leqslant(\Delta+\hbar \omega)$.[49] Excess energy creates thermal disequilibrium in the material, characterized by equation 1.4 . When the deviation of quasi-particle occupation probability distribution in the normal state $\left(f_{k}\right)$ is made negative, through quasi-particle reduction within the two-fluid model, $\delta T^{*}$ becomes negative. [49] Thus, the effective temperature is reduced and the energy gap is widened. The effective temperature is a thermodynamic parameter, not an actual temperature.

S11 deviations between preliminary simulations and testing appeared, upon reassessment of the expected results, in the parameters of sheet impedance and chip geometry. The simulated sheet impedance was assumed as a normal state value, but the actual device function was coupling into a zero-resistance superfluid state. 


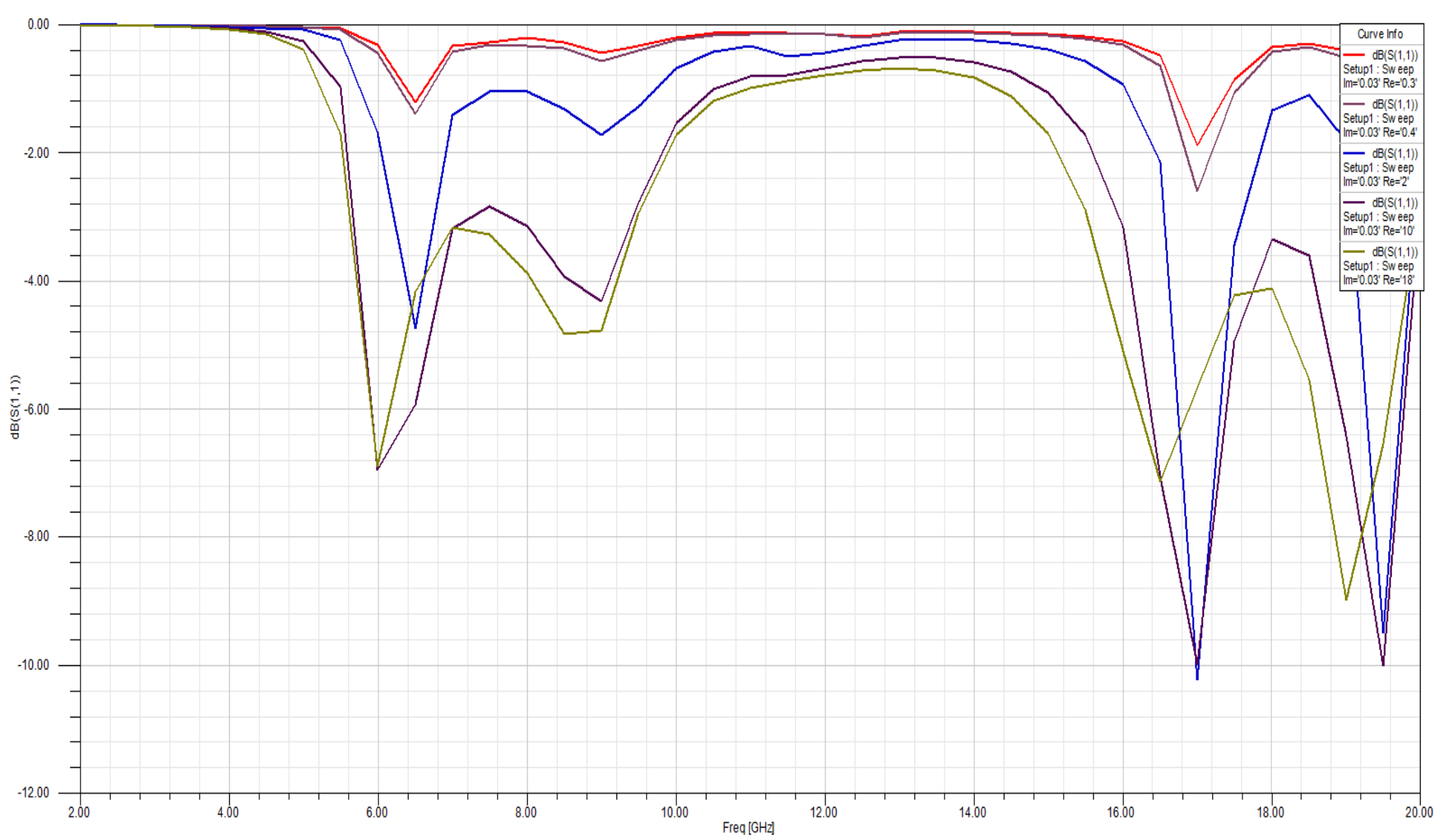

Figure 6.4: Sheet impedance parameter sweeps, accounting for the reduced material impedance in the superconducting state. The reduced material impedance creates a higher S11. These sweeps were produced with an adjusted quartz chip, which led to coupling at low frequency levels. 
Adjusted HFSS simulations showed deviations in S11 dips with varied sheet impedance of the $\mathrm{Nb}$, as shown in Figure 6.4. Importantly, the $\mathrm{S} 11$ is much higher for low sheet impedance values. This plot incorporates the full chip dimensions, as illustrated in Figures 6.1 and 6.2 , as well as a cryogenic-adjusted quartz dielectric constant ${ }^{23}$ The functional changes in the quartz chip properties created resonances, leading to local minima in S11 near the observed evanescent coupling frequencies.

\subsection{Abrikosov Flux-Flow Resistance}

Evanescent modes supported within the dielectric led to the development of a flux-flow resistance, which was both easily observed and manipulable.

\subsubsection{Development of Dielectric Resonance}

An eigenmode evaluation in HFSS revealed peak resonances of $4.5 \mathrm{GHz}, 5.3 \mathrm{GHz}$, and 8.9GHz; all consistent with the observed evanescent mode dominant frequencies during testing. The HFSS-generated Q values for these resonances were all in the range of 100 . These low Q values were not surprising, given the fact that the dielectric substrate was designed to avoid resonance. Rather than acting like a traditional dielectric resonator, the quartz had assumed a resonant-like operation due to signal reflections, which built a magnetic field across it.

\footnotetext{
${ }^{23}$ The original simulation used $\epsilon_{r}=3.8$. This value was adjusted to 4.5 for post testing simulations; then swept to account frequency dependence sensitivity.
} 
Considerations to avoid dielectric resonance were nullified by interaction between the receive antenna and frequencies with $\lambda_{Q t z}$ comparable to the $\sim 20 G H z \lambda_{g}$ in a vacuum. The wavelength through quartz at dominant frequency, $4 \mathrm{GHz}$, is approximately $35.5 \mathrm{~mm}$, assuming a $4.2 \mathrm{~K}$ quartz dielectric constant of 4.5 . At $9 \mathrm{GHz}, \lambda_{(Q t z)}$ is $15.7 \mathrm{~mm}$. These wavelengths are respectively comparable to $\lambda_{g} / 4$ and $\lambda_{g} / 2$ in the slot antenna, based on the original design frequency. A small error margin exists with the dielectric constant value assumption, due to frequency-dependent polarization of the quartz.[2] The effect of the quartz dielectric was essentially wavelength conditioning of low frequencies to couple into the modified slot antenna. Coupling was further enhanced by the extension of the transmit coax farther into the waveguide.

A post testing HFSS parametric sweep was performed to assess deviations in ACC function caused by monopole extension alterations. Results are illustrated in Figure 6.5. Notable in this plot is that longer extensions of the monopole within the waveguide allow minimal S11 at reduced frequency levels, as was observed during testing. 


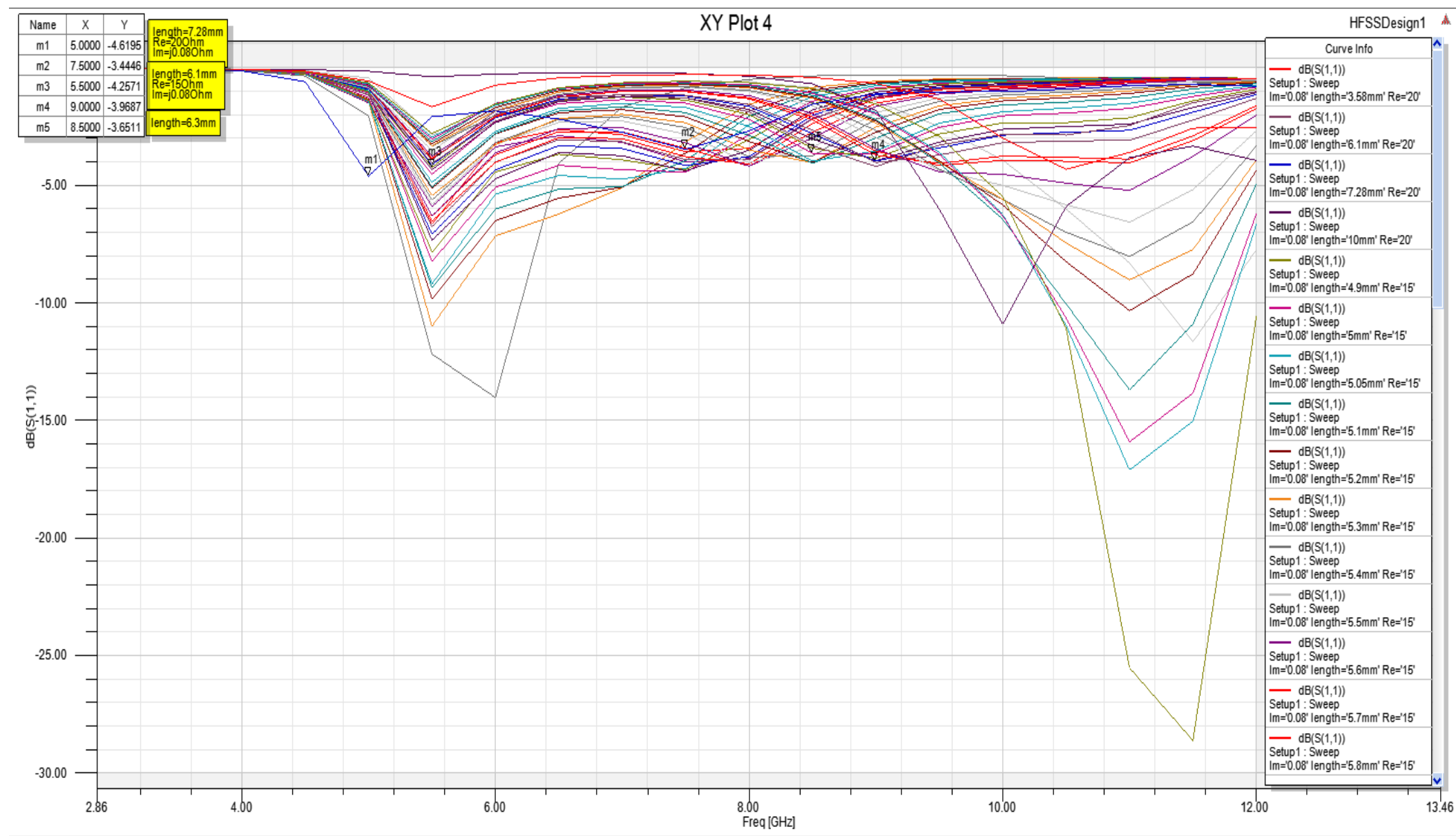

Figure 6.5: Sequential parametric sweeps of the transmit sleeve monopole extension length confirm that placements allowing longer transmit segments within the waveguide cause low S11 at reduced frequency levels. 

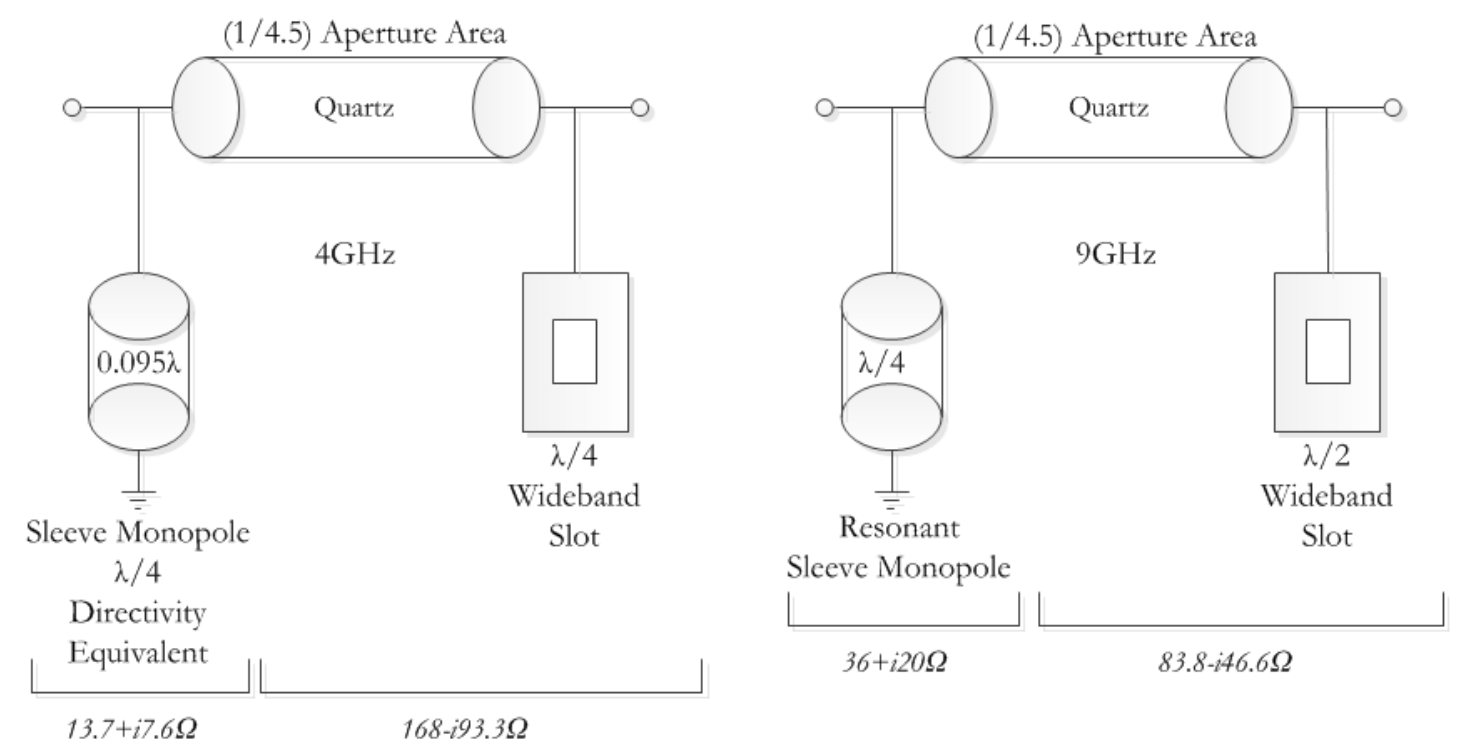

Figure 6.6: Equivalent circuit schematics and functional values at the observed induced voltage peaks of $4 G H z$ and $9 G H z$.

The most significant voltage rise occurred with a total coaxial extension into the waveguide of $7.28 \mathrm{~mm}$. This juxtaposition created a sleeve monopole antenna, where the total extension into the waveguide creates the effective transmission length.[3, 47] Sleeve antennas consist of an inner transmit radiator with outer conductive sheathing, and act like two asymmetrically driven antennae.[3] They are commonly made from coaxial cables and allow increases in bandwidth over traditional wire antennas.[47] Sleeve monopoles posses radiation resonance at $\lambda / 4$. [47] The $7.28 \mathrm{~mm}$ sleeve was approximately equivalent to $0.095 \lambda$ at $4 G H z$ and $\lambda / 4$ at $9 G H z$, which indicates directivity and resonance of a quarter-wave monopole for both frequencies ${ }^{24}$ At $4 G H z$, the transmit impedance was significantly smaller than that of $9 \mathrm{GHz}$, creating a more drastic mismatch with the slot and inducing a stronger reflected field within the quartz. Figure 6.6 shows the equivalent circuit values at

\footnotetext{
${ }^{24}$ For a $50 \Omega$ RF transmit cable.
} 
both $4 G H z$ and $9 G H z$, which were assessed using variations of equation 2.10 and Smith chart tracking. Similar radiation dynamics occurred at the $5.15 \mathrm{~mm}$ coax extension into the waveguide, with transmit attenuation resulting from less effective quarter-wave monopole matching.

A dielectric slot-line $\lambda_{(Q t z)} / 4$ high-field receive antenna developed at $4 G H z$; and a dielectric resonator, induced by $\lambda_{(Q t z)} / 2$ slot-line antenna, was formed at $9 G H z$. Traditional dielectric resonance was not supported at either of these frequencies, as the longitudinal $\beta_{(Q t z)}$ was imaginary for both. Rather, the quartz and slot presented an impedance transformer, which changed functional qualities of both. [8, 11] Necessity of the slot antenna was confirmed by contrast of the thin $1.15 \mathrm{~mm}$ strip-line test, which did not show any significant induced voltage with RF application.

Though the waveguide did not support frequencies below $17.58 \mathrm{GHz}$, the quartz dielectric allowed their interaction with the slot receive antenna through its functional adherence to qualities of evanescent mode waveguide filters.[18] Evanescent modes below the waveguide cut-off were not subject to constraints of guided wavelengths because they were inherently bound to the dielectric. Through the impedance transformation, the dielectric was able to store select fields like a filter. Effective slot aperture ratios for all evanescent frequencies were identical, at $22 \%$, caused by inverse proportionality to the dielectric constant. 


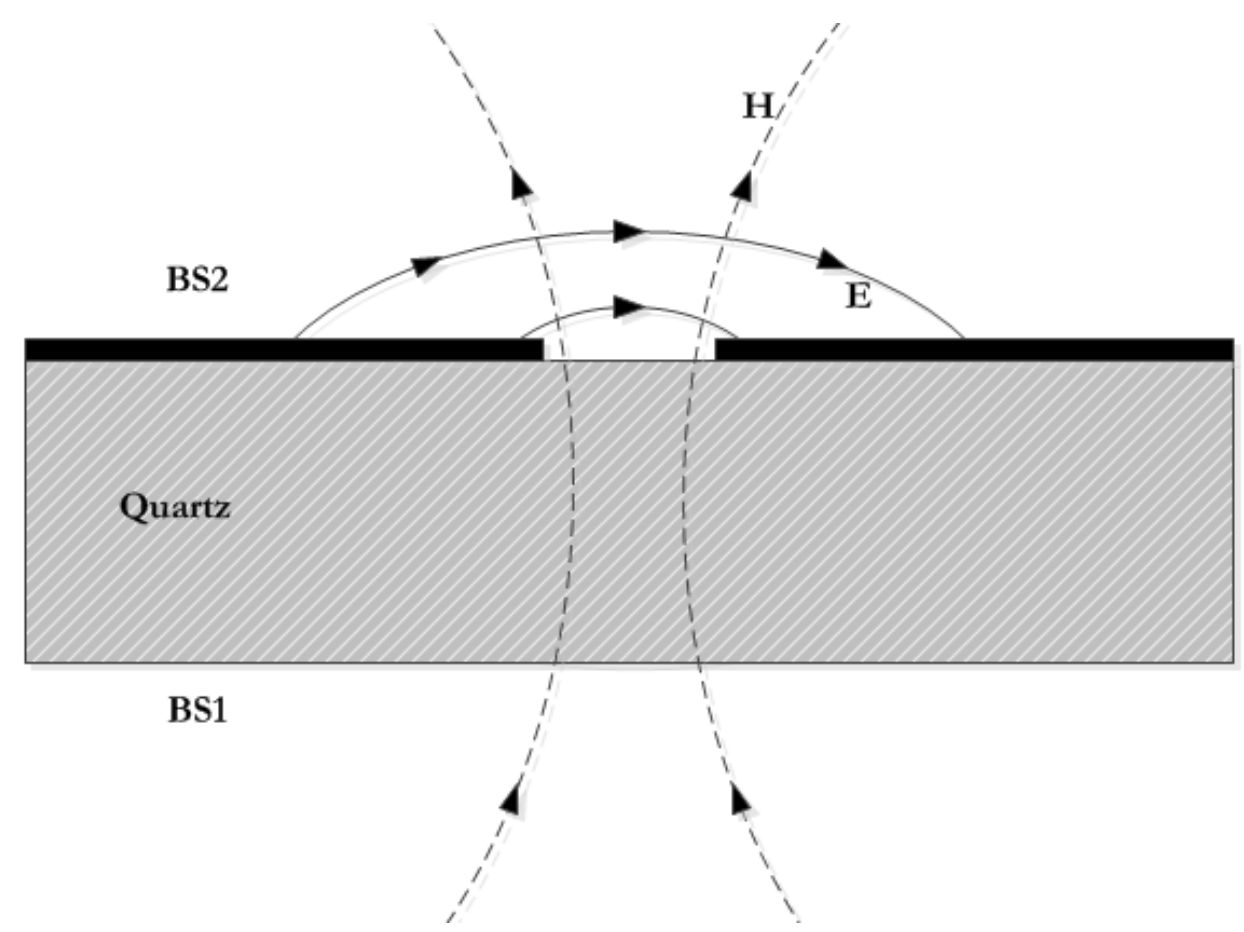

Figure 6.7: Field representation of the slot-line effect on the quartz dielectric for evanescent mode resonance.

The cavity filter reaction of the quartz created an elliptical magnetic field normal to the thin-film surface, with field geometry shown in Figure 6.7, [7, 8, 11, 18] The largest field was created at $4 G H z$ as the slot presented a $\lambda_{(Q t z)} / 4$ impedance with high transmit-receive mismatch, resulting from the electrically small monopole antenna.

Work done by [8] explains slot-line induced dielectric resonance to be established with high dielectric materials (dielectric constant $>10$ ). ACC results attained with quartz show that the resonance-supportive dielectric constant value can be reduced significantly through tuned slot dimensions. This shows a trade-off between antenna size and dielectric value. 


\subsubsection{Abrikosov Vortex}

In the presence of a perpendicular magnetic field, very thin film type I superconductors have been found to exhibit type II behavior, energetically favoring a mixed normal/superconducting domain.[23, 49] It occurs as a result of comparable spatial parameters of the magnetic flux penetration depth and material thickness. The threshold qualifier for this behavior is a coherence length of the film which is less than its thickness. [23, 49] Referring back to Figure 1.1, the combination of these conditions creates a scenario where the type I model assumes the functional properties of a type II.

The Ginzburg-Landau (GL) coherence length defines a distance over which cooper-pairs maintain homogeneous phase through deviations in the pseudo-wave energy function.[49] In this design, it is the characteristic spatial length of a cooper-pair. Standard coherence length assessment for pure $\mathrm{Nb}$ samples is approximately 38nm.[46] However, according to Ginzburg-Landau theory, coherence length varies with temperature, in the following relation. [49]

$$
\xi(T)=\frac{\hbar}{\left|2 m^{*} \alpha(T)\right|^{1 / 2}}
$$

where,

$\hbar=$ Planck's constant

$m^{*}=$ Particle mass in quantum - mechanical current

$\alpha(T)=$ Thermal Cooper - pair breaking strength 
This is a secondary relation, with $\alpha(T)$ as the intermediary. The functional relation between GL coherence length and temperature is of direct proportionality. [49] As the temperature approaches zero, the coherence length is reduced, and within sufficiently reduced range from $T_{C}$ crosses below the $\mathrm{Nb}$ deposition thickness of $\sim 30 \mathrm{~nm}$. This is consistent with testing observations, as the evanescent mode voltage development was suppressed at sufficiently low temperatures (below $\sim 7 K$ ).

Temperature biasing also dictates the critical magnetic field to disturb superconductivity.

$$
H_{C}(T) \approx H_{C}(0)\left[1-\left(\frac{T}{T_{C}}\right)^{2}\right]
$$

Equation 6.2 illustrates a reduction of the required critical magnetic field level at temperatures approaching $T_{C}$. This also affects a lengthening of the magnetic flux penetration depth, further mimicking type II behavior as shown in Figure 1.1, [23, 49] When the temperature bias of the ACC was in the range of $7 K-9.2 K$, it functioned as a type II superconductor, where both superconductivity and magnetic penetration were allowed. Essentially, the Meissner effect, creating diamagnetism in the material, was mitigated.

Another factor dictating the temperature dependence of flux-flow voltages relates to the Bean-Livingston barrier, which defines the free energy required for Abrikosov vortex development.[19, 49] When the temperature is sufficiently low, the free energy in the material is reduced to a point that cannot support traditional means of flux-flow creation. However, the material can be forced to develop a voltage through flux-lattice rejection or absorption in the presence of respectively low or high perpendicular magnetic fields. Very 
low magnetic fields induce a surface flux-flow from the rejection, and very high magnetic fields overcome the Meissner effect to force flux de-pinning.[19]

\subsubsection{Flux-flow Resistive Voltage}

The presence of flux penetration within the $\mathrm{Nb}$ engendered an additional element to the device, namely the ability for said flux to induce a resistive voltage. An energy threshold existed for observing the $\sim 6 m V$ reversible voltage. This appeared as a jump, rather than the steady voltage increase expected of quasi-particle redistribution. Similar behavior of voltage spikes at an energy threshold have been observed for flux-flow in $\mathrm{Nb}$ foils, which was shown by [23]. Flux-flow can occur in both type I and type II superconductors, producing resistive elements is both. [23, 49] Due to the strong evidence that the ACC Nb layer behaved as a type II superconductor in the applied testing temperature bias region, flux-flow resistance is discussed in terms of type II superconductivity.

During type II superconductivity of an Abrikosov vortex state, the domain width between the superconducting and normal species in reduced. The energy associated with this boundary contributes to the material free energy.[23] Magnetic flux then penetrates more easily into the superfluid. The magnetic flux is contained in flux lines, which combine to form a lattice. The flux lattice is held in place by a pinning force, which effectively contains the flux and keeps it separate from the superfluid.[23, 49] When a current is applied during this condition, it introduces a Lorentz force perpendicular to the pinning force. In the case that the Lorentz current force is larger than the pinning force the flux lattice becomes unstable and is de-pinned, moving in a direction opposite the pinning force and perpendicular 
to both the applied current and magnetic field. Resultant from this motion are eddy currents around the normal-state particles, which create a resistive voltage.[23] This process was the culprit in the observed evanescent mode induced voltages of the ACC.

In steady-state, on a macroscopic scale, the flux-flow voltage adheres to Faraday's Law.[23] The flux magnitude is a product of current density, applied field and material viscosity coefficient.[23, 49] It contains two components, which become dynamic in transitory intervals. These components are known as the resistive voltage and the Hall voltage. The angle of the transverse electric field, which causes voltage, is called the Hall angle and it dictates the resistance of the developed voltage.[23] If the Hall angle creates a longitudinally directed electric field, the voltage will not be resistive.[23, 49] A future research inquiry on this particular voltage development exists in designing experimental inputs to specify a desired Hall angle. This becomes more complex in the consideration of voltage-cancelling superconducting domain flow, which is beyond the scope of this project, but should be addressed in future iterations of the ACC.[23] 


\section{Chapter 7: Conclusion}

In proof-of-concept experimentation for a switching mechanism using quasi-particle redistribution, a novel alternate method involving flux-flow resistive voltage was discovered. From this has arisen insight on the superconductive device function, which will inform next stage design goals.

\subsection{Findings}

Conclusions, drawn from the major findings, are asserted in this section.

\subsubsection{Quasi-Particle Redistribution}

The zero-resistance superfluid response to RF perturbations was not significant, and moreover, required an excessively long time interval for development. Competing semiconductor switching technology actuates on the scale of $\mu s$, while the slight voltage development in the quasi-particle redistribution experiments arose after $\sim 1 s$. Future work involving this switching mechanism would demand close attention to the transition timing.

Testing reflected induced voltage levels that were neither adequately large nor controllable for ease in switching implementation. Furthermore, because voltages developed in both the applied and absent RF scenarios, with respective deviations in timing, the observed quasi-particle redistribution effect was related more to energy gap changes than induced 
voltages within the superconducting state. These findings do not facilitate objective proof to reject the theory of quasi-particle redistribution as a potential switching mechanism. However, further investigations into the phenomenon must include a host of new considerations on biasing conditions and RF energy levels, based on lessons from this design.

\subsubsection{Flux-Flow}

Type II biasing of the ACC created an Abrikosov vortex mixed domain state, which carried a controllable induced voltage. This occurred below the functional design frequency of the device, a phenomenon which was supported by dielectric loading between the transmit and receive antennae. Flux-flow caused resistance, which was found to contain a manipulable vortex lattice. This property of flux-flow within type II superconductors created new engineering questions on material science and quantum process design for optimized voltage development.

\subsection{Further Work}

Further work is assessed, in references to lessons and new inquiries garnered from the results of the initial testing process.

\subsubsection{Quasi-Particle Disruption Experimentation}

The ACC design frequency was set low in the $\mathrm{Nb}$ disruption bandwidth due to output constraints imposed by the employed signal generator. Further investigation into the effects of quasi-particle redistribution could incorporate a VNA to achieve a frequency output 
closer to the high end of the bandwidth, at $120 \mathrm{GHz}$. This would require new antenna and waveguide dimensional design. It would also create higher insertion loss within the RF cable, thus requiring more input power. Common VNAs output an approximate maximum power of $10 \mathrm{dBm}$. Bearing this in mind, tests run at higher frequencies may necessitate an auxiliary power amplification element.

\subsubsection{Flux-Flow Engineering}

Immediate work stemming from the experimental results of this project involves the creation of models for quasi-particle scattering parameters and effective quantum activity; as well as characterization of Abrikosov development and destruction under the specific testing conditions. The latter can serve to inform future design constraints for flux-flow-induced switching operations. Also, wound wires could be placed within the waveguide to establish a perpendicular magnetic field at the design frequency for analyzing flux lattice de-pinning activity at $19.8 \mathrm{GHz}$.

In light of the type II superconducting flux-flow voltage, several engineering questions have arisen. The resistive voltage was not significant in terms of mitigating a large portion of the superfluid. Further work would address useful implementation, as well as optimization, of flux-flow voltages. The introduction of a resistive element within the mixed state could be utilized in power flow redirection, or in spurring a complete transition in an appropriately designed device.

General upscale for enhanced power handling capability of future ACC valves will be mainly an effort in materials assessment. The Abrikosov vortex within a thin film 
can be substituted with a thicker film of a traditional type II superconductor. This would allow higher current levels through the device, without sacrificing the partial magnetic flux penetration required for a flux-flow voltage. Also, traditional type II superconductors adopt the superconducting state at much higher temperatures than type I. Use of type II material would allow cooling with liquid nitrogen, rather than helium; a change which is advantageous in both expense and availability of the natural resource.

Incorporating a ceramic type II superconductor into future designs may be an advantageous option, since engineering a full transition to the normal state would create an electrical insulator of the material. For switching, a dramatic change in metered resistance is desired. Considerations toward materials assessment have inspired a design method to optimize dielectric resonance in creating a perpendicular magnetic field on a type II ceramic superconductor. Rather than designing the dielectric substrate to avoid resonance, it can be utilized to promote it. This would require surveys on appropriate dielectrics, in terms of relative permittivity and temperature stability. Many designs incorporating dielectric resonators at cryogenic temperatures employ either silicon or sapphire. [28, 33, 50] As seen in the deviations of ACC results between amorphous and crystalline quartz samples, nuances of the material lattice must also be addressed in specification of a resonant dielectric.

Patterned on the resonator would be a thin layer of normal metal with a dielectric-loaded patch antenna. The normal metal would serve to provide a predictable non-zero sheet impedance and couple RF radiation across the dielectric, thus producing the perpendicular magnetic field with the aforementioned slot-line effect.[7, 8, 11] This normal metal layer 
would serve only to provide a patch antenna; it would not provide a parallel current path. Directly above the slot antenna would be a ceramic type II superconducting substrate, which would be flux-destabilized through the magnetic field that is supported by the dielectric and patch antenna below. Another option would be to place the ceramic superconducting substrate in the center of the resonant dielectric for maximum field exposure.

Interfacing between dissimilar materials would need to be addressed in order to avoid RF signal reflection, superconducting Andreev reflection, thermal deformation, and galvanic effects. The design constraints for this new device iteration would include an element of timing for both the development of a perpendicular magnetic field in the resonator, as well as the flux penetration into the superconductor. The timing for a full voltage development can be engineered through the energy balance equation for magnetic field penetration within the materials.[23] Beyond this, next generation ACC valves would need to incorporate control strategies for current and temperature biasing in order to obtain optimal voltage development. 


\section{Bibliography}

[1] K. B. Alici and E. Ozbay. Electrically small split ring resonator antennas. Journal of Applied Physics, 101(8):083104, April 2007.

[2] I.V. Anisimkin, Yu.V. Gulyaev, V.I. Anisimkin, and E. Verona. Temperature sensitivity of plate modes in st quartz. In Ultrasonics Symposium, 2001 IEEE, volume 1, pages 423-426 vol.1, 2001.

[3] Balanis. Antenna Theory Analysis and Design. Harper and Row.

[4] Robert Bass. Portland State University, 2013.

[5] Greg Baty. Portland State University, 2013.

[6] Berkeley. Resonance in acoustic tubes. Web, 2013.

[7] A. Buerkle and K. Sarabandi. A wide-band, circularly polarized, magnetodielectric resonator antenna. Antennas and Propagation, IEEE Transactions on, 53(11):34363442, Nov 2005.

[8] Amelia Buerkle, Kamal Sarabandi, and Hossein Mosallaei. Compact slot and dielectric resonator antenna with dual-resonance, broadband characteristics. IEEE TRANSACTIONS ON ANTENNAS AND PROPAGATION, 53(3):1020-1027, March 2005. 
[9] BYU. Wet Chemical Etching of Metals and Semiconductors - Niobium. Web.

[10] Richard Campbell. Portland State University, 2013.

[11] Seymour B. Cohn. Slot line on a dielectric substrate. Microwave Theory and Techniques, IEEE Transactions on, 17(10):768-778, Oct 1969.

[12] Lakeshore Cryotronics. Quad-Twist ${ }^{\mathrm{TM}}$ cryogenic wire - QT-32, QT-36. Web.

[13] Lakeshore Cryotronics. Semi-Rigid Coaxial Cable - Type SR. Web.

[14] Lakeshore Cryotronics. Specifications: DT-670. Web.

[15] Aaron Datesman. NASA, 2013.

[16] Aric Datesman. Collaborator - machinist, 2013.

[17] The Free Dictionary. Dendrite. Web.

[18] S.J. Fiedziuszko. Dual-mode dielectric resonator loaded cavity filters. Microwave Theory and Techniques, IEEE Transactions on, 30(9):1311-1316, Sep 1982.

[19] Stenuit G., Michotte S., Govaerts J., and Piraux L. Temperature dependence of penetration and coherence lengths in lead nanowires. Superconductor Science and Technology, 18(1), 2005.

[20] Zygmunt Galasiewicz. Superconductivity and Quantum Fluids. International Series of Monographs in Natural Philosophy.

[21] Georgia Institute of Technology. Photolithography. Web. 
[22] J. W. Hafstrom and M. L. A. MacVicar. Case for a second energy gap in superconducting niobium. Phys. Rev. B, 2:4511-4516, Dec 1970.

[23] R.P. Huebener. Magnetic Flux Structures in Superconductors. Springer.

[24] South Day Technology Inc. Comparing wax layer thickness after mounting. Web.

[25] Technics INC. Technics 800 SERIES MICRO RIE SYSTEM Operation And Maintenance Manual. Web, Jan. 2010.

[26] Inc. Keithley Instruments. Series 2400 SourceMeter ${ }^{\circledR}$ SMU Instruments. Web.

[27] Inc. Keithley Instruments. Device Characterization Techniques using Keithley SourceMeter ${ }^{\circledR}$ Instruments with LabTracer Software. Web, 2002.

[28] J. Krupka, J. Breeze, N.McN. Alford, A.E. Centeno, L. Jensen, and T. Claussen. Measurements of permittivity and dielectric loss tangent of high resistivity float zone silicon at microwave frequencies. In Microwaves, Radar Wireless Communications, 2006. MIKON 2006. International Conference on, pages 1097-1100, May 2006.

[29] Brookhaven National Laboratory. Properties of helium. Web.

[30] Arthur Lichtenberger. University of Virginia, 2013.

[31] A.W. Lichtenberger, D.M. Lea, and F.L. Lloyd. Investigation of etching techniques for superconductive nb/al-al/sub 2/o/sub 3//nb fabrication processes. Applied Superconductivity, IEEE Transactions on, 3(1):2191-2196, 1993. 
[32] Sungkyun Lim and Hao Ling. A two-element, electrically small yagi antenna. In Antennas and Propagation Society International Symposium, 2007 IEEE, pages 24552458, June 2007.

[33] A.G. Mann, A.N. Luiten, D.G. Blair, and M. J. Buckingham. Ultrastable cryogenic sapphire dielectric microwave resonators. In Frequency Control Symposium, 1992. 46th., Proceedings of the 1992 IEEE, pages 167-171, May 1992.

[34] MicroChemicals. Gold Etching. Web, Nov. 2013.

[35] Inside Mines. RCA clean. Web.

[36] Achmad Munir and Muhammad Fathi Yakan Musthofa. Rectangular to circular waveguide converter for microwave devices characterization. International Journal on Electrical Engineering and Informatics, 3(3):147-157, 2011.

[37] V. Novotny and P.P.M. Meincke. Single superconducting energy gap in pure niobium. Journal of Low Temperature Physics, 18(1-2):147-157, 1975.

[38] United States Department of Energy. Fy 2015 congressional budget request: Advanced research projects agency - energy. Web.

[39] V. Palmieri and et. al. Besides the standard niobium bath chemical polishing. The 10th Workshop on RF Superconductivity, 2001.

[40] Branimir Pejcinovic. Portland State University, 2013.

[41] D.M. Pozar. Microwave Engineering. John Wiley and Sons, 2 edition, 1998. 
[42] M. Raskovic, J. Upadhyay, and et.al. Plasma etching rates and surface composition of bulk niobium treated in ar/cl2 microwave discharge. In Proceedings of EPAC08, Genoa, Italy, pages 928-930, 2008.

[43] Rohm and Haas Electronic Materials. Megaposit ${ }^{\mathrm{TM}}$ SPR $^{\mathrm{TM}} 220$ series photoresists. Web, 2004.

[44] Jay Sasserath. Dry etching of niobium using ccl2f2 and cf4: A comparison. Journal of Applied Physics, 68, 1990.

[45] Six Sigma. Ionic cleanliness testing. Web.

[46] Soltan Soltan. Interaction of Superconductivity and Ferromagnetism in YBCO-LCMO Heterostructures. Cuvillier Verlag.

[47] Warren Stutzman and Gary Thiele. Antenna Theory and Design. John Wiley and Sons.

[48] Agilent Technologies. Agilent E8241A/44A/51A/54A PSG Family Performance Signal Generator Data Sheet. Web.

[49] Michael Tinkham. Introduction to Superconductivity. McGraw-Hill Inc.

[50] M.E. Tobar, J. Krupka, J.G. Hartnett, E.N. Ivanov, and R.A. Woode. High-q sapphirerutile frequency-temperature compensated microwave dielectric resonators. Ultrasonics, Ferroelectrics and Frequency Control, IEEE Transactions on, 45(3):830-836, May 1998.

[51] Tontechnik-Rechner. Open and Closed Tubes. Web. 
[52] Portland State University. PSU PNNA developer recipe.

[53] T. Van Duzer and C.W. Turner. Principals of Superconductive Devices and Circuits. Elsevier.

[54] Patrick Verdonck. Plasma etching. Web.

[55] Phillip Wong. Portland State University, 2013. 


\section{Appendix A: Deposition Process Steps}

This process was developed with the intention of performing evaporation and deposition using equipment and facilities at PSU. In the case that future work is done with these materials, this process algorithm will be used.

Note: Perform evaporation at Pressure $\leqq 10^{-6}$ Torr

1. RCA Clean

2. Sputter $\sim 30 \mathrm{~nm} \mathrm{Nb}$

- Ensure $\mathrm{N}_{2}$ is on (for purge) (close after done)

- Go to 'Platen'

- Door opens at platen position \#3 ('Home' at platen position \#1)

To open chamber (head A)

- PC - Process Chamber

- To Vent - 'Start PC Vent'

- LL - Load Lock

Plasma (Platen 2)

- Has manual shutter

- Turn on $\mathrm{O}_{2}$ (close later)

- Turn on forward drive motor stage out

- Open shutter

- Go to Depo

- Turn on 'ion source gas'

- Turn on 'ion source power supply' - check VSI

- Run 1 min

- Off power supply 
- Close shutter

$-\mathrm{O}_{2}$ off

Process Note:

* Normal $\mathrm{p} \sim 10^{-4}-10^{-5}$ range

* Must be $<10^{-3}$

* Less filament damage

$\mathrm{Nb}$ Sputter

- 5Q5-242 to Nb Sputter (Assuming Target Replacement)

- Platen 5, Ar on (turn off later) (4 \& 5 share a power supply)

- PC High Vac Throttle on (Vacuum menu) $\rightarrow$ (throttle cryo pump)

- Burn Nb target (getters too)

- Pick Power Supply:

- Deposition Menu: Source SWS, set gas (gas menu)

- Ar Mode $4 \rightarrow$ gas injector on, SMT (SCCR will settle around 21)

To Deposition Menu

- Close shutter

- Power supply 4 on

- Ramp rate to $3 \mathrm{U} / \mathrm{s}$

- Power set to 300W

- Check for stable plasma

- Run for a few minutes to clean head

- Close shutter

- Rotate to Platen 5

- Zero crystal (SQS - 242 software)

- Depth: Open Shutter

Units Note:

* 100 Angstrom; $0.1 \mathrm{k} \AA=10 \mathrm{~nm}$

- Close shutter

- Turn off: 3U/s to OW

- Power Supply off

- Source SWS off

- Gas off: Mode 0, SCCM 0, close gas injector

- Turn off throttle 
3. Evaporate $50 \mathrm{~nm} \mathrm{Au}$

- Platen 1 (SQS - 242) to Au evap.

- To warm-up, set stage to Platen 4 (to shield)

- Evap. shutter open (manual), clock-wise

- Bottom shutter open (to see mirror)

- Ensure vacuum $<10^{-6}-10^{-7}$ range

- Turn on E-beam power supply

- Set EB off to on (Depo Menu)

- Breaker on

- Warm-up for $20 \mathrm{~min}$ (3申, Main power)

- Turn on scan controller (Telemark)

- Connect remote cask to sweep control box

- EB off to off

- EB on to on

- Pneumatic shutter open

- Ramp rate to $1 \mathrm{U} / \mathrm{s}$

- Set point to $6 \%$

- Center beam, turn on 'triangle'

- Ramp set point to $10 \%$ (depends on depo rate)

- Check beam, re-adjust if needed

- Ramp set until rate is $\sim 1-5 \AA / \mathrm{sec}$

Process notes:

* 100nm, $1000 \AA$

* Choose correct crucible (in this case it was 4)

- Set point $16 \%$ (rate $\sim 8 \AA / \mathrm{s}$ ) (this is a bit aggressive, $\sim 15 \%$ may be better)

- Rotate to position 1

- Hit 'FWD' to turn on rotation

- Zero sensor, open E-beam shutter and re-zero sensor (as fast as possible)

- Observe thickness, close E-beam shutter

End Process

- Ramp down to $5 \%$, in steps of $1 \%$

- Move to position 4

- Ramp to $0 \%$, in steps of $1 \%$ 
- EB on to off

- EB off to on

- Disconnect E-beam controller and put back in panel

- Take out through load lock (after a few minutes of cooling)

- Go to vacuum

- Start LL pump

- Load lock finishes, choose 'OK'

Start Sample transfer

- Ignore 'Paused' prompt

- Go to position 6

- Take sample out (don't open chamber)

- Choose 'Continue' on 'Paused' prompt, choose 'OK' on following menu

- Start LL Vent

- Wait several minutes 


\section{Appendix B: Au Patterning Process Steps}

Au photolithography and etching followed the outlined steps below.

Preliminary Steps:

1. Mix Etchant - $\mathrm{KI}: \mathrm{I}_{2}: \mathrm{H}_{2} \mathrm{O}(4 \mathrm{~g}: 1 \mathrm{~g}: 80 \mathrm{~mL})[4,34]$

2. Mix developer - 0.2 Molar concentration, $\mathrm{NaOH}: \mathrm{H}_{2} \mathrm{O}(2.4 \mathrm{~g}: 300 \mathrm{~mL})$

3. Turn lithography lamp on for warm-up and test settings

- Power switch

- Hold start button until the lamp lights

- Lamp warm-up time is $5 \mathrm{~min}$

- Set expose time to $60 \mathrm{sec}$

RCA Organic Clean

1. Qtip IPA (Preliminary degrease)

2. IPA/MeOH/IPA spin clean (Organic contaminants and degrease)

Preparation

1. $100^{\circ} \mathrm{C}$ Hot Plate Bake 5min (Drives off moisture)

Photoresist Application

1. Spin Photoresist SPR220-3 - 2krpm, 60sec for approximate thickness of $4 \mu \mathrm{m}$ [43]

- 400RPM, $10 \mathrm{sec}, 3$ Ramp unit

- 2000RPM, 60 sec, 1 Ramp unit

- End

- Pause $>4 \mathrm{~min}$ (Room temperature slow bake to settle material and allow material smoothing.)

- $100^{\circ} \mathrm{C} \mathrm{HPB} 60 \mathrm{sec}$ - cure (Prebake to drive off excess photoresist solvent, typically at $90^{\circ} \mathrm{C}-100^{\circ} \mathrm{C}$ for $30-60$ seconds on a hotplate.) 
- Pause $2 \min$ (Calm and settle surface)

Edge Bead Removal (This step was omitted during processing.)

1. Expose with ACC00 Burn-off mask (edge bead), 120sec, Direct UV

2. Develop $\mathrm{NaOH}: \mathrm{H}_{2} \mathrm{O}(2.4 \mathrm{~g}: 300 \mathrm{~mL}) \mathrm{t}=60-120 \mathrm{sec}$ (Watch)

3. Deionized water (DI) rinse

Exposure and Developing

1. Expose with ACC01 Au mask, 60sec, Direct UV

2. $100^{\circ} \mathrm{C}$ PEB $120 \mathrm{sec}$ (Post Exposure Bake, firms and smooths side walls of resist.)

3. Develop $\mathrm{NaOH}: \mathrm{H}_{2} \mathrm{O}(2.4 \mathrm{~g}: 300 \mathrm{~mL}) \mathrm{t}=20 \mathrm{sec}($ Watch$)$

4. DI rinse

Etching

1. Etch $-\mathrm{KI}: \mathrm{I}_{2}: \mathrm{H}_{2} \mathrm{O}(4 \mathrm{~g}: 1 \mathrm{~g}: 80 \mathrm{~mL}), \mathrm{dx} / \mathrm{dt} \approx 1 \mu \mathrm{m} / \mathrm{min}$ for $\mathrm{Au}[34]$

2. $\mathrm{t}=5 \mathrm{sec}+$ check $+5 \mathrm{sec}+$ check $+5 \mathrm{sec}$

Photoresist Removal [4]

1. Acetone/MeOH/IPA

2. MeOH Qtip

3. Acetone/MeOH/IPA

4. DI rinse/IPA

Shut Down

1. Contact Lithograph

- Remove mask

- Turn off power supply

- Turn off all vacuum switches

- Turn off microscope lamp

- Turn off timer

- Set equipment back to original position

2. Wet Station 
- Turn off hotplate

- Turn off spinner (in back of machine)

- Turn off main wet station hood power

- Return solvents to original locations

- Dump developer in waste contamination vessel

- Rinse glassware and leave upside down in sink

3. Clean Room

- Turn off vacuum valve \#3

- Turn off DC power supply and master switch

- Throw away trash

- Return chemicals to outer room hood

- Sign out in lab log and record time 


\section{Appendix C: HF Handling Safety for Nb Etch Procedures}

Because HF dissolves glass, all beakers, tweezers, funnels, measurement flasks and handling equipment were made of plastic. A specialized lab and fume hood were used in HF etching processes. The fume hood contained a constant vacuum and back liquid scrubber to quickly remove any HF from the working environment. The hood was drawn to a near-fully-closed position, allowing only enough clearance for an arm to reach into the workspace.

In avoidance of chemical and corrosive flesh burns, four layers of gloves were worn during chemical handling. The bottom layer was a thin nitrile glove, covered with silvershield gloves, which were then covered with thick neoprene gloves. A tube PVC rubber glove (without hand enclosure) was then used to cover the contact point between sleeve end and glove entrance. Body protection was provided by a Tyvek jumpsuit, worn over a full-cover layer of clothing. This was covered by a rubber apron. Facial exposure was prevented through the use of a rigid plastic face mask and plastic goggles.

Post etching procedures included disposal of waste liquid into plastic waste bottles, which were taped on the seal. All working surfaces and protective attire were then sprayed with $10 \% \mathrm{NaOH}$ neutralizing solution and wiped clean with absorbent pads. Non-liquid waste was disposed in a sealed plastic waste bin. 
After removal of protective attire and upon exit of the HF lab, Calgonate gel was applied to any possible exposed skin (face and hands) as a preemptive measure. Work was performed with a partner waiting outside the HF lab, whom had been informed of dangers listed on the HF MSDS.

In the case of exposure or contamination, a spill kit was stored in the HF lab. Contact information was obtained before using the HF lab for hazardous poison control officials, as well as the lab safety manager. 


\section{Appendix D: Nb RIE Process Algorithm}

The following itemized steps detail Nb RIE etching, and assume that photolithography has been completed on the sample.

Initialize

1. Outside clean room, turn on cooling platter

2. Open valve on oxygen tank (Front tank near wet bench, only need to open a small amount.)

3. Open valve on $\mathrm{CF}_{4}$ tank (Small tank next to RIE.)

4. Open valve on Nitrogen tank (Back tank near wet bench, only need to open a small amount.)

5. Plug into power strip 4 things

- RIE (Large black cable with round head.)

- Pressure meter for chamber (Black box.)

- Pump (Large grey cable.)

- Shinko Pirani Guage (Smaller grey cable.)

Start Prep

1. Open chamber

- Inspect Platen

2. Load wafer if comfortable (Best to do a dry run first.)

3. Power switch on (Back upper right of RIE.)

- All lights should come on

4. Make sure all toggle switches on bottom and center faces of RIE are down except Torr. These include: 
- Bottom Face

- Mode: Manual

- Vac: Closed

- Power: Off

- Gas \#1: Off

- Gas \#2: Off

- Center Face

- $\mathrm{O}_{2}$ : Down

$-\mathrm{CF}_{4}$ : Down

- AFC: Up

- Gas 1: Up

Process

1. Look at pressure on Shinko, should be 20mTorr or less (No gasses on pump side.)

2. Set Vac to 'Open' (Pressure will spike to $\sim 8.2$ Torr, wait until it goes back down to $\sim 30$ mTorr or less.)

3. Set upper right dial on center face to 'Set'

4. Set Gasses A and B to desired sccm (In this case it was $6 \mathrm{sccm}$ for $\mathrm{O}_{2}$ and $47 \mathrm{sccm}$ for $\mathrm{CF}_{4}$.)

- Go back to 'Read' (It should be at zero, or the offset of the machine.)

5. Toggle A and B up in the center face

6. Toggle Gas \#1 'On' in the bottom face

- A and B LED indicator lights will be on (Upper left in the center face.)

7. After the gasses are running, pressure should be 300 mTorr $+/-15 \%$

\section{Light}

1. Turn the POT next to the power switch on the bottom face to the far left (ie: turn all the way down.)

2. Flip the power toggle to 'On'

3. Get ready with timer, watch, gas and power

4. After power is on, increase until it reads $100 \mathrm{~W}$ on the upper right of RIE

- It will light around 30W. Go fairly quickly because it is actively etching once lit. 
Etch

In this case 11 min at specified levels will etch $\sim 30 \mathrm{~nm}$ of $\mathrm{Nb}$.

End

1. Toggle power 'Off'

- Turn POT to full left (Off position.)

2. Gas \#1 'Off' in bottom face

3. Toggle A Down in center face $\left(\mathrm{O}_{2}\right)$

4. Toggle B Down in center face $\left(\mathrm{CF}_{4}\right)$

5. Toggle Vent 'Open' in bottom face (Wait 60 seconds.)

6. Toggle Vac 'Closed' in bottom face (Wait until chamber can be opened.)

- Continue venting

- Un-plug pump

- Close valves on $\mathrm{O}_{2}$ and $\mathrm{CF}_{4}$ (Keep nitrogen open while venting.)

7. Remove and inspect wafer

Shut Down

1. Toggle Vent 'Closed'

2. Close RIE Front panels

3. Turn off RIE

4. Close valve on nitrogen

5. Turn off Shinko

6. Turn off cooling platter

7. Turn off power strip next to RIE 


\section{Appendix E: Temperature Calibration}

Due to unanticipated pressure levels within the LHe dewar, the assumed full submersion temperature was raised $\sim 2 K$ above the expected value. Initially, this was compensated by adjusting the bias current in the temperature sensor to retain the expected temperature. This created unreliable temperature data recordings, which were subsequently adjusted for accuracy.

Manufacturer-provided temperature translations from voltage readings of the DT670ABO sensor were based on a $10 \mu A$ input, but $10.5 \mu A$ was applied during testing. Assuming that lattice interactions within the DT670A-BO diode retain specific energy allowance per temperature level, adjustments were made based on power level profiles. This was done in the range of temperatures below $27 K$, where data recordings were made. The DT670A-BO sensor has a very high temperature sensitivity in environments below $30 \mathrm{~K}$, with an error range of $15 \mathrm{mV} / \mathrm{K}-37 \mathrm{mV} / \mathrm{K}$. The expected error margin in temperature recording at the tested levels is a maximum of $0.5 \mathrm{~K}$. This pertains to systematic accuracy of all readings. Voltage readings were interpreted with a cubic spline function in MATLAB $^{\circledR}$ to find corresponding temperature levels. Spreadsheet values for the current biasing error correction are shown below. 
Voltage for $10.5 \mathrm{~A}$ bias temp sensor, assuming fixed $\mathrm{R}$ per temperature

\begin{tabular}{|c|c|c|c|c|c|}
\hline Temp & $\mathrm{V}(10 \mathrm{uA})$ & Pwr (10uA) & $\operatorname{Pwr}(10.5 u A)$ & Rtemp (10uA) & $V(10.5 u A)$ \\
\hline 1.4 & 1.64429 & $1.64429 \mathrm{E}-05$ & $1.7265 \mathrm{E}-05$ & 164429 & 1.7265045 \\
\hline 1.5 & 1.64299 & $1.64299 \mathrm{E}-05$ & $1.72514 \mathrm{E}-05$ & 164299 & 1.7251395 \\
\hline 1.6 & 1.64157 & $1.64157 \mathrm{E}-05$ & $1.72365 \mathrm{E}-05$ & 164157 & 1.7236485 \\
\hline 1.7 & 1.64003 & $1.64003 \mathrm{E}-05$ & $1.72203 \mathrm{E}-05$ & 164003 & 1.7220315 \\
\hline 1.8 & 1.63837 & $1.63837 \mathrm{E}-05$ & $1.72029 \mathrm{E}-05$ & 163837 & 1.7202885 \\
\hline 1.9 & 1.6366 & 0.000016366 & $1.71843 \mathrm{E}-05$ & 163660 & 1.7184300 \\
\hline 2 & 1.63472 & $1.63472 \mathrm{E}-05$ & $1.71646 \mathrm{E}-05$ & 163472 & 1.7164560 \\
\hline 2.1 & 1.63274 & $1.63274 \mathrm{E}-05$ & $1.71438 \mathrm{E}-05$ & 163274 & 1.7143770 \\
\hline 2.2 & 1.63067 & $1.63067 \mathrm{E}-05$ & $1.7122 \mathrm{E}-05$ & 163067 & 1.7122035 \\
\hline 2.3 & 1.62852 & $1.62852 \mathrm{E}-05$ & $1.70995 \mathrm{E}-05$ & 162852 & 1.7099460 \\
\hline 2.4 & 1.62629 & $1.62629 \mathrm{E}-05$ & $1.7076 \mathrm{E}-05$ & 162629 & 1.7076045 \\
\hline 2.5 & 1.624 & 0.00001624 & 0.000017052 & 162400 & 1.7052000 \\
\hline 2.6 & 1.62166 & $1.62166 \mathrm{E}-05$ & $1.70274 \mathrm{E}-05$ & 162166 & 1.7027430 \\
\hline 2.7 & 1.61928 & $1.61928 \mathrm{E}-05$ & $1.70024 \mathrm{E}-05$ & 161928 & 1.7002440 \\
\hline 2.8 & 1.61687 & $1.61687 \mathrm{E}-05$ & $1.69771 \mathrm{E}-05$ & 161687 & 1.6977135 \\
\hline 2.9 & 1.61445 & $1.61445 \mathrm{E}-05$ & $1.69517 \mathrm{E}-05$ & 161445 & 1.6951725 \\
\hline 3 & 1.612 & 0.00001612 & 0.000016926 & 161200 & 1.6926000 \\
\hline 3.1 & 1.60951 & $1.60951 \mathrm{E}-05$ & $1.68999 \mathrm{E}-05$ & 160951 & 1.6899855 \\
\hline 3.2 & 1.60697 & $1.60697 \mathrm{E}-05$ & $1.68732 \mathrm{E}-05$ & 160697 & 1.6873185 \\
\hline 3.3 & 1.60438 & $1.60438 \mathrm{E}-05$ & $1.6846 \mathrm{E}-05$ & 160438 & 1.6845990 \\
\hline 3.4 & 1.60173 & $1.60173 \mathrm{E}-05$ & $1.68182 \mathrm{E}-05$ & 160173 & 1.6818165 \\
\hline 3.5 & 1.59902 & $1.59902 \mathrm{E}-05$ & $1.67897 \mathrm{E}-05$ & 159902 & 1.6789710 \\
\hline 3.6 & 1.59626 & $1.59626 \mathrm{E}-05$ & $1.67607 \mathrm{E}-05$ & 159626 & 1.6760730 \\
\hline 3.7 & 1.59344 & $1.59344 \mathrm{E}-05$ & $1.67311 \mathrm{E}-05$ & 159344 & 1.6731120 \\
\hline 3.8 & 1.59057 & $1.59057 \mathrm{E}-05$ & $1.6701 \mathrm{E}-05$ & 159057 & 1.6700985 \\
\hline 3.9 & 1.58764 & $1.58764 \mathrm{E}-05$ & $1.66702 \mathrm{E}-05$ & 158764 & 1.6670220 \\
\hline 4 & 1.58465 & $1.58465 \mathrm{E}-05$ & $1.66388 \mathrm{E}-05$ & 158465 & 1.6638825 \\
\hline 4.2 & 1.57848 & $1.57848 \mathrm{E}-05$ & $1.6574 \mathrm{E}-05$ & 157848 & $\begin{array}{r}1.6574040 \\
1.6570\end{array}$ \\
\hline 4.4 & 1.57202 & $1.57202 \mathrm{E}-05$ & $1.65062 \mathrm{E}-05$ & 157202 & 1.6506210 \\
\hline 4.6 & 1.56533 & $1.56533 \mathrm{E}-05$ & $1.6436 \mathrm{E}-05$ & 156533 & 1.6435965 \\
\hline 4.8 & 1.55845 & $1.55845 \mathrm{E}-05$ & $1.63637 \mathrm{E}-05$ & 155845 & 1.6363725 \\
\hline 5 & 1.55145 & $1.55145 \mathrm{E}-05$ & $1.62902 \mathrm{E}-05$ & 155145 & 1.6290225 \\
\hline 5.2 & 1.54436 & $1.54436 \mathrm{E}-05$ & $1.62158 \mathrm{E}-05$ & 154436 & 1.6215780 \\
\hline 5.4 & 1.53721 & $1.53721 \mathrm{E}-05$ & $1.61407 \mathrm{E}-05$ & 153721 & 1.6140705 \\
\hline 5.6 & 1.53 & 0.0000153 & 0.000016065 & 153000 & 1.6065000 \\
\hline 5.8 & 1.52273 & $1.52273 \mathrm{E}-05$ & $1.59887 \mathrm{E}-05$ & 152273 & 1.5988665 \\
\hline
\end{tabular}




\begin{tabular}{|c|c|c|c|c|c|}
\hline Temp & $\mathrm{V}(10 \mathrm{uA})$ & Pwr (10uA) & $\operatorname{Pwr}(10.5 u A)$ & Rtemp (10uA) & $\mathrm{V}(10.5 \mathrm{uA})$ \\
\hline 6 & 1.51541 & $1.51541 \mathrm{E}-05$ & $1.59118 \mathrm{E}-05$ & 151541 & 1.5911805 \\
\hline 6.5 & 1.49698 & $1.49698 \mathrm{E}-05$ & $1.57183 \mathrm{E}-05$ & 149698 & 1.5718290 \\
\hline 7 & 1.47868 & $1.47868 \mathrm{E}-05$ & $1.55261 \mathrm{E}-05$ & 147868 & 1.5526140 \\
\hline 7.5 & 1.46086 & $1.46086 \mathrm{E}-05$ & $1.5339 \mathrm{E}-05$ & 146086 & 1.5339030 \\
\hline 8 & 1.44374 & $1.44374 \mathrm{E}-05$ & $1.51593 \mathrm{E}-05$ & 144374 & 1.5159270 \\
\hline 8.5 & 1.42747 & $1.42747 \mathrm{E}-05$ & $1.49884 \mathrm{E}-05$ & 142747 & 1.4988435 \\
\hline 9 & 1.41207 & $1.41207 \mathrm{E}-05$ & $1.48267 \mathrm{E}-05$ & 141207 & 1.4826735 \\
\hline 9.5 & 1.39751 & $1.39751 \mathrm{E}-05$ & $1.46739 \mathrm{E}-05$ & 139751 & 1.4673855 \\
\hline 10 & 1.38373 & $1.38373 \mathrm{E}-05$ & $1.45292 \mathrm{E}-05$ & 138373 & 1.4529165 \\
\hline 10.5 & 1.37065 & $1.37065 \mathrm{E}-05$ & $1.43918 \mathrm{E}-05$ & 137065 & 1.4391825 \\
\hline 11 & 1.3582 & 0.000013582 & $1.42611 \mathrm{E}-05$ & 135820 & 1.4261100 \\
\hline 11.5 & 1.34632 & $1.34632 \mathrm{E}-05$ & $1.41364 \mathrm{E}-05$ & 134632 & 1.4136360 \\
\hline 12 & 1.33499 & $1.33499 \mathrm{E}-05$ & $1.40174 \mathrm{E}-05$ & 133499 & 1.4017395 \\
\hline 12.5 & 1.32416 & $1.32416 \mathrm{E}-05$ & $1.39037 \mathrm{E}-05$ & 132416 & 1.3903680 \\
\hline 13 & 1.31381 & $1.31381 \mathrm{E}-05$ & $1.3795 \mathrm{E}-05$ & 131381 & 1.3795005 \\
\hline 13.5 & 1.3039 & 0.000013039 & $1.3691 \mathrm{E}-05$ & 130390 & 1.3690950 \\
\hline 14 & 1.29439 & $1.29439 \mathrm{E}-05$ & $1.35911 \mathrm{E}-05$ & 129439 & 1.3591095 \\
\hline 14.5 & 1.28526 & $1.28526 \mathrm{E}-05$ & $1.34952 \mathrm{E}-05$ & 128526 & 1.3495230 \\
\hline 15 & 1.27645 & $1.27645 \mathrm{E}-05$ & $1.34027 \mathrm{E}-05$ & 127645 & 1.3402725 \\
\hline 15.5 & 1.26794 & $1.26794 \mathrm{E}-05$ & $1.33134 \mathrm{E}-05$ & 126794 & 1.3313370 \\
\hline 16 & 1.25967 & $1.25967 \mathrm{E}-05$ & $1.32265 \mathrm{E}-05$ & 125967 & 1.3226535 \\
\hline 16.5 & 1.25161 & $1.25161 \mathrm{E}-05$ & $1.31419 \mathrm{E}-05$ & 125161 & $\begin{array}{r}1.3141905 \\
1.19\end{array}$ \\
\hline 17 & 1.24372 & $1.24372 \mathrm{E}-05$ & $1.30591 \mathrm{E}-05$ & 124372 & 1.3059060 \\
\hline 17.5 & 1.23596 & $1.23596 \mathrm{E}-05$ & $1.29776 \mathrm{E}-05$ & 123596 & 1.2977580 \\
\hline 18 & 1.2283 & 0.000012283 & $1.28972 \mathrm{E}-05$ & 122830 & 1.2897150 \\
\hline 18.5 & 1.2207 & 0.000012207 & $1.28174 \mathrm{E}-05$ & 122070 & 1.2817350 \\
\hline 19 & 1.21311 & $1.21311 \mathrm{E}-05$ & $1.27377 \mathrm{E}-05$ & 121311 & 1.2737655 \\
\hline 19.5 & 1.20548 & $1.20548 \mathrm{E}-05$ & $1.26575 \mathrm{E}-05$ & 120548 & 1.2657540 \\
\hline 20 & 1.197748 & $1.19775 \mathrm{E}-05$ & $1.25764 \mathrm{E}-05$ & 119774.8 & 1.2576354 \\
\hline 21 & 1.181548 & $1.18155 \mathrm{E}-05$ & $1.24063 \mathrm{E}-05$ & 118154.8 & 1.2406254 \\
\hline 22 & 1.162797 & $1.1628 \mathrm{E}-05$ & $1.22094 \mathrm{E}-05$ & 116279.7 & 1.2209369 \\
\hline 23 & 1.140817 & $1.14082 \mathrm{E}-05$ & $1.19786 \mathrm{E}-05$ & 114081.7 & 1.1978579 \\
\hline 24 & 1.125923 & $1.12592 \mathrm{E}-05$ & $1.18222 \mathrm{E}-05$ & 112592.3 & 1.1822192 \\
\hline 25 & 1.119448 & $1.11945 \mathrm{E}-05$ & $1.17542 \mathrm{E}-05$ & 111944.8 & 1.1754204 \\
\hline 26 & 1.115658 & $1.11566 \mathrm{E}-05$ & $1.17144 \mathrm{E}-05$ & 111565.8 & 1.1714409 \\
\hline 27 & 1.11281 & $1.11281 \mathrm{E}-05$ & $1.16845 \mathrm{E}-05$ & 111281 & 1.1684505 \\
\hline
\end{tabular}




\section{Appendix F: Thin Strip-Line Current Sweep Temperature Depression}

Figures F.1 and F.2 show current sweep comparisons from zero through $I_{C}$ for the comparative antenna versus thin $1.15 \mathrm{~mm}$ strip-line tests. The antenna results were consistent with expectations, in terms of the temperature profile for increasing current levels. The current sweep illustrates slight evidence of gap enhancement when exposed to these testing conditions, but with a human reaction time error margin that calls any conclusion on gap effects into question.

The surprising information in comparing these two samples exists primarily in the temperature profile of the thin strip-line, which decreased with initial increases in current level. In terms of induced voltage, Figure F.2 shows almost identical induced voltage with RF on and RF off (with exception of the last data point), but the temperature levels were very different and charted a dip in mid current sweep. These results, along with the small temperature drop at the end of the sweep with RF on, were repeated in four separate current sweep tests.

Cooling of type II superconductors has been noted in the presence of a changing magnetic field near the highest critical field transition point. [23, 49] This could be an effect of transition within the metal, enacted by the strength of a magnetic flux circulating around the thin strip-line. If this were the case; while the strip-line acted like a type I at low current levels, a 
transverse hall component of induced flux dominated, which affected temperature but not resistive voltage. When a small amount of energy was fed into the metal from the applied current, quasi-particles may have been elevated in a gap-enhancement mode, which could produce cooling. Enhancement then continued until the applied current was sufficiently large to affect a mixed flux-flow regime. At this point the temperature began to rise and a voltage developed.

An alternative explanation involves Andreev reflection at the interface between $\mathrm{Nb}$ and Au. Thermal effects on either side of the interface may have been isolated within respective metals, and therefore altered the energy balance to affect cooling on the superconducting side.

In terms of current density, the B-TL-0 thin strip-line carried $J_{C} \sim 1.4 e 10 \mathrm{~A} / \mathrm{m}^{2}$ and the B-MS-FT chip carried $J_{C} \sim 2.3 e 9 \mathrm{~A} / \mathrm{m}^{2}$. So, counter to expectations, the thin strip-line carried a larger current density at the transition point.

These phenomena require further inquiry for comprehensive assessment, and are included here only as compelling observations. 

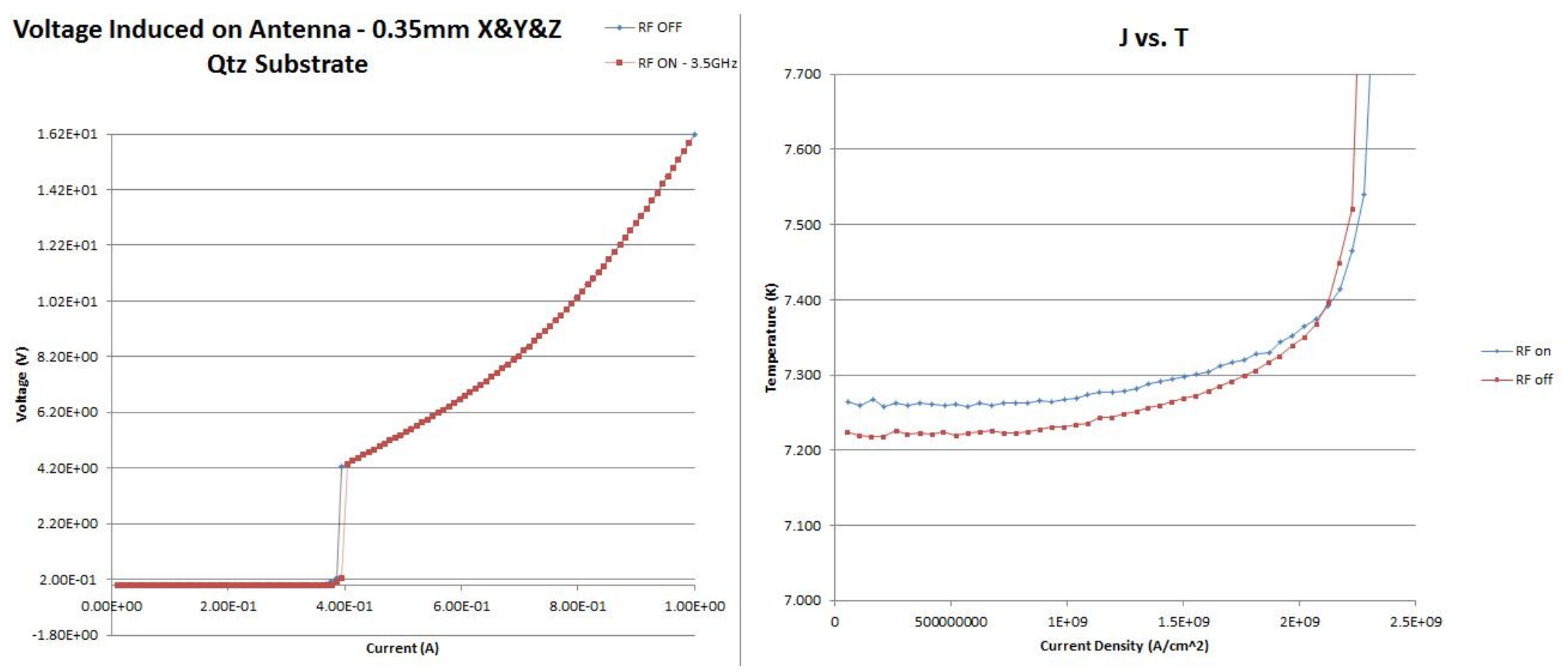

Figure F.1: Current sweep voltage and temperature profiles for B-MS-FT, showing trends consistent with standard expectations. 
Voltage Induced on Thin Nb Wire $-0.35 \mathrm{~mm}$ X\&Y\&Z Qtz Substrate

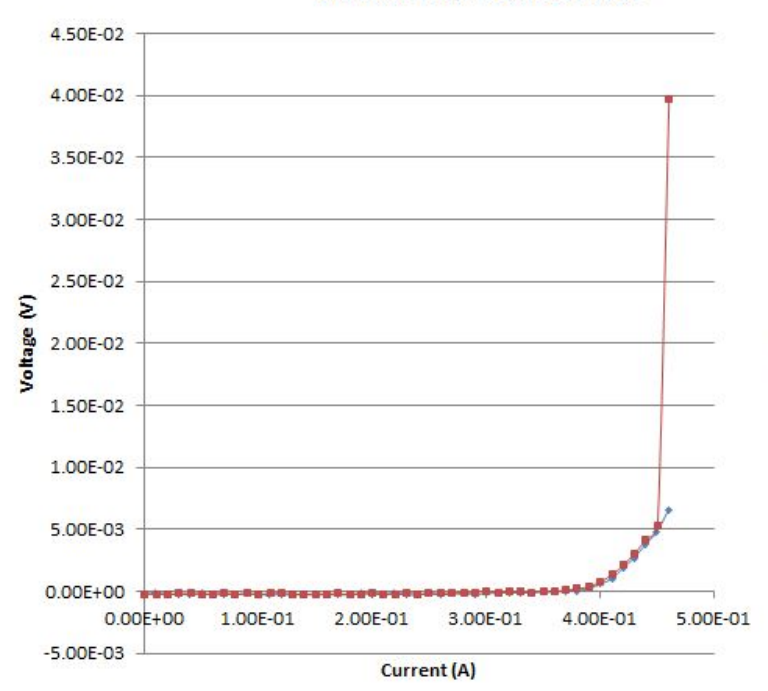

J vs. T

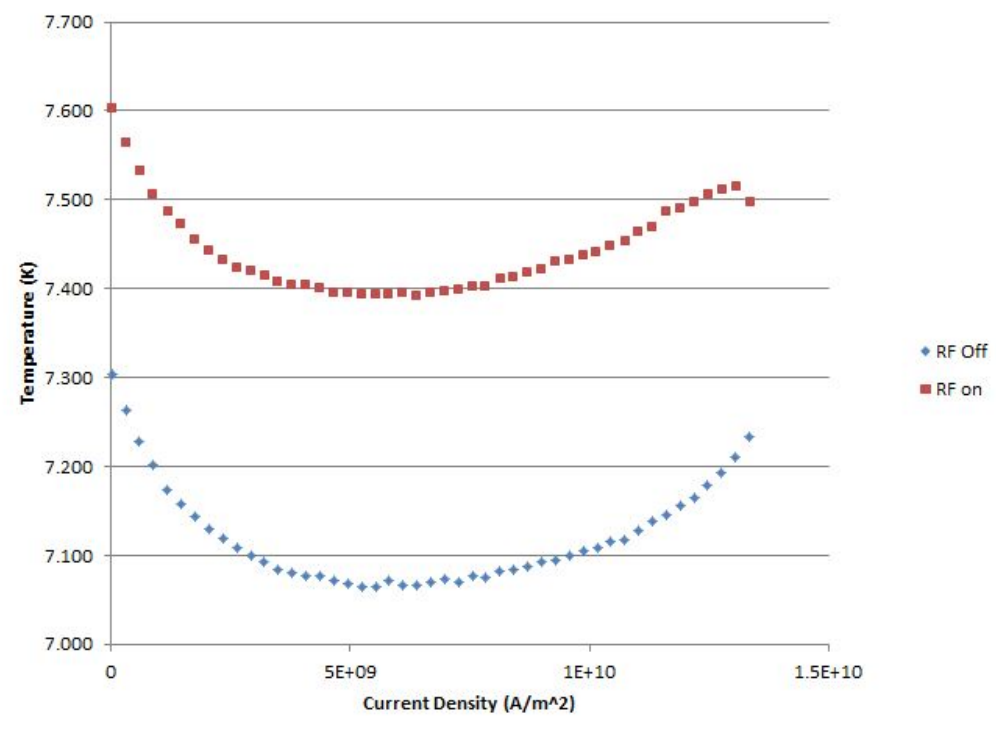

Figure F.2: Current sweep voltage and temperature profiles for B-TL-0, showing wire cooling with increased applied current; as well as a current density level far surpassing expectations. 